\title{
Quantum Hamilton mechanics: Hamilton equations of quantum motion, origin of quantum operators, and proof of quantization axiom
}

\author{
Ciann-Dong Yang * \\ Department of Aeronautics and Astronautics, National Cheng Kung University, Tainan 701, Taiwan \\ Received 14 January 2006; accepted 24 July 2006 \\ Available online 8 September 2006
}

\begin{abstract}
This paper gives a thorough investigation on formulating and solving quantum problems by extended analytical mechanics that extends canonical variables to complex domain. With this complex extension, we show that quantum mechanics becomes a part of analytical mechanics and hence can be treated integrally with classical mechanics. Complex canonical variables are governed by Hamilton equations of motion, which can be derived naturally from Schrödinger equation. Using complex canonical variables, a formal proof of the quantization axiom $\mathbf{p} \rightarrow \hat{\mathbf{p}}=-\mathrm{i} \hbar \nabla$, which is the kernel in constructing quantum-mechanical systems, becomes a one-line corollary of Hamilton mechanics. The derivation of quantum operators from Hamilton mechanics is coordinate independent and thus allows us to derive quantum operators directly under any coordinate system without transforming back to Cartesian coordinates. Besides deriving quantum operators, we also show that the various prominent quantum effects, such as quantization, tunneling, atomic shell structure, Aharonov-Bohm effect, and spin, all have the root in Hamilton mechanics and can be described entirely by Hamilton equations of motion.
\end{abstract}

Keywords: Hamilton mechanics; Complex extension; Quantum motion; Quantum operator; Quantization axiom

\section{Quantum Hamilton mechanics}

Quantum mechanics had been established from the following two postulates:

\footnotetext{
* Fax: +886 62389940.

E-mail address: cdyang@mail.ncku.edu.tw
} 
(1) Postulate of correspondence: to any self-consistent and well-defined observables $A$, there corresponds an operator $\hat{A}$.

(2) Postulate of quantization: the operator $\hat{A}$ corresponds to the observable $A(\mathbf{q}, \mathbf{p})$ can be constructed by replacing the coordinate $\mathbf{q}$ and momentum $\mathbf{p}$ in the expression for $A$ by the assigned operators $\mathbf{q} \rightarrow \hat{\mathbf{q}}=\mathbf{q}$ and $\mathbf{p} \rightarrow \hat{\mathbf{p}}=-\mathrm{i} \hbar \nabla$.

The validity of the two postulates was justified indirectly via the voluminous precise predictions of quantum mechanics. Although the two postulates work very successfully, until now we still do not know why they should work and a formal proof of their origin still lacks. We also do not know very clearly about the underlying reason that to obtain the correct operators in coordinate system other than Cartesian coordinates, it is always necessary to transform $A(\mathbf{q}, \mathbf{p})$ into Cartesian coordinates before putting in the operators. This paper aims to prove the two postulates by the first principle of Hamilton mechanics, to expound the reason why the postulate of quantization is only true in the Cartesian coordinates, to demonstrate how to obtain directly quantum operators in curvilinear coordinates without transforming back to Cartesian coordinates, and to describe major quantum effects by Hamilton equations of motion.

The main idea of the paper is based on the equivalence between an observable $A(\mathbf{q}, \mathbf{p})$ in Hamilton mechanics and its associated operator $\hat{A}$ in quantum mechanics, where $(\mathbf{q}, \mathbf{p})$ are canonical variables defined in complex domain. Based on this equivalence and the requirement that the behavior of $A(\mathbf{q}, \mathbf{p})$ must obey Hamilton equations, we can determine the expression and the various quantization properties of $\hat{A}$ directly from Hamilton equations of motion, regardless of the coordinate system being used. The Hamilton equations considered here are derived from a quantum Hamiltonian $H$, which is different from the classical one. To find out the correct quantum Hamiltonian, we first recall a classical result that for a given classical Hamiltonian $H_{\mathrm{c}}(t, \mathbf{q}, \mathbf{p})$, the classical Hamilton-Jacobi (H-J) equation reads

$$
\frac{\partial S_{\mathrm{c}}}{\partial t}+\left.H_{\mathrm{c}}(t, \mathbf{q}, \mathbf{p})\right|_{\mathbf{p}=\nabla S_{\mathrm{c}}}=0
$$

where $S_{\mathrm{c}}$ is the classical action function. We may regard the classical H-J equation as the short wavelength limit of Schrödinger equation [1]:

$$
\mathrm{i} \hbar \frac{\partial \psi}{\partial t}=-\frac{\hbar^{2}}{2 m} \nabla^{2} \psi+V \psi,
$$

as can be seen via the following transformation

$$
\psi=\exp (\mathrm{i} S / \hbar),
$$

from which Schrödinger equation becomes

$$
\frac{\partial S}{\partial t}+\left[\frac{1}{2 m}(\nabla S)^{2}+V\right]=\frac{\mathrm{i} \hbar}{2 m} \nabla^{2} S .
$$

We recognize the quantity in brackets as the classical Hamiltonian for a single particle described in Cartesian coordinates. Eq. (1.4) is known as the quantum $\mathrm{H}-\mathrm{J}$ equation, which reduces to the classical $\mathrm{H}-\mathrm{J}$ equation (1.1) if the right-hand side of Eq. (1.4) is negligible, which means that the wavelength of the matter wave is so short that the momentum changes by a negligible fraction over a distance of wavelength [1]. The transformation 
(1.3) was first introduced by Schrödinger in transforming the phase function $\phi=S / h$ governed by Fresnel's wave equation to the wavefunction $\psi$ governed by Schrödinger's wave equation.

If we treat Eq. (1.4) as the quantum-mechanical counterpart of the classical H-J equation (1.1), it is natural to ask what will be the corresponding quantum-mechanical counterpart of the classical Hamiltonian $H_{\mathrm{c}}$. Rewriting Eq. (1.4) in a form analogous to Eq. (1.1):

$$
\frac{\partial S}{\partial t}+\left.H(t, \mathbf{q}, \mathbf{p})\right|_{\mathbf{p}=\nabla S}=0
$$

we obtain the desired quantum Hamiltonian $H$, compatible with Schrödinger equation, as

$$
H(\psi)=\frac{1}{2 m} \mathbf{p}^{2}+V(\mathbf{q})+Q(\psi(\mathbf{q})),
$$

where $Q$ is known as quantum potential defined by

$$
Q(\psi(\mathbf{q}))=\frac{\hbar}{2 m \mathrm{i}} \nabla \cdot \mathbf{p}=\frac{\hbar}{2 m \mathrm{i}} \nabla^{2} S=-\frac{\hbar^{2}}{2 m} \nabla^{2} \ln \psi(\mathbf{q}) .
$$

The usage of the notation $H(\psi)$ is to emphasize the state-dependent nature of the quantum Hamiltonian $H$. The classical Hamiltonian $H_{\mathrm{c}}$ depends only on the externally applied potential $V(\mathbf{q})$, whereas the quantum Hamiltonian $H(\psi)$ depends on $V(\mathbf{q})$ as well as on the internal state $\psi$ where the particle lies. For a given state described by $\psi(\mathbf{q})$, the quantum Hamiltonian $H(\psi)$ defined in Eq. (1.6) is an explicit function of the canonical variables $\mathbf{q}$ and $\mathbf{p}$ that are regarded as independent variables. There are two roles played by the wavefunction $\psi$ in the quantum Hamiltonian $H$. First, as indicated in Eq. (1.5), it determines the canonical momentum $p_{j}$ according to

$$
p_{j}=\frac{\partial S}{\partial q_{j}}=-\mathrm{i} \hbar \frac{\partial \ln \psi}{\partial q_{j}} .
$$

Second, it generates the quantum potential $Q$ according to Eq. (1.7). The equations of motion for a particle moving in the quantum state $\psi$ are derived by applying the quantum Hamiltonian $H$ to the Hamilton equations:

$$
\begin{aligned}
& \frac{\mathrm{d} \mathbf{q}}{\mathrm{d} t}=\frac{\partial H(\psi)}{\partial \mathbf{p}}=\frac{1}{m} \mathbf{p}, \\
& \frac{\mathrm{d} \mathbf{p}}{\mathrm{d} t}=-\frac{\partial H(\psi)}{\partial \mathbf{q}}=-\frac{\partial}{\partial \mathbf{q}}\left[V(\mathbf{q})-\frac{\hbar^{2}}{2 m} \nabla^{2} \ln \psi(\mathbf{q})\right] .
\end{aligned}
$$

Note that as in classical Hamilton mechanics, we have obtained $\partial H / \partial \mathbf{p}$ and $\partial H / \partial \mathbf{q}$ by treating $\mathbf{q}$ and $\mathbf{p}$ as independent variables in the Hamiltonian $H$ in Eq. (1.6). The Hamilton equations (1.9) are distinct from the classical ones in two aspects: the complex nature and the state-dependent nature. The complex nature is a consequence of the fact that the canonical variables (q, p) solved from Eqs. (1.8) and (1.9) are, in general, complex variables. The state-dependent nature means that the Hamilton equations of motion (1.9) govern the quantum motion exclusively in the specific quantum state described by $\psi$. We will show in the subsequent sections that all the quantum operators and the various quantum effects can be derived from the complex canonical momentum in Eq. (1.8) and from the complex-extended Hamilton equations of motion in Eq. (1.9). 
In the next section, we first discuss the meanings of a wavefunction in Hamilton mechanics by pointing out that each wavefunction $\psi$ represents a dynamic system described by Eq. (1.9). Hence, information contained in $\psi$ can be extracted from the dynamic system (1.9) using methods developed in analytical mechanics. Section 3 establishes the relationship between a complex observable $A(\mathbf{q}, \mathbf{p})$ in Hamilton mechanics and its associated operator $\hat{A}$ in quantum mechanics. This relationship allows us to derive any operator $\hat{A}$ from its counterpart complex function $A(\mathbf{q}, \mathbf{p})$ in Hamilton mechanics and to express the commutator $[\hat{A}, \hat{B}]$ in terms of the Poisson bracket $\{A, B\}$ in Hamilton mechanics. In Section 4, we take harmonic oscillator as a typical example to demonstrate how a quantum problem can be analyzed in the framework of Hamilton mechanics. Tunneling is a motion in classically prohibited region, which cannot be treated by using classical mechanics. However, if we extend canonical variables to complex domain, classically prohibited region no longer exists and the entire tunneling trajectory can be described by using Hamilton equations of motion as will be shown in Section 5. Hamilton equations of motion (1.9) are valid only for Cartesian coordinates. In Section 6, we derive quantum operators and Hamilton equations of motion in spherical coordinates, based on which we solve quantum central-force problems, such as the electron motion in hydrogen atom, by Hamilton mechanics. Quantum operators in general curvilinear coordinates and in the presence of electromagnetic field are derived from the Hamilton equations of motion in Sections 7 and 8, respectively; in the meanwhile, the spin quantization is proved and its geometrical interpretation is addressed according to the Hamilton equations of motion.

\section{Dynamical representation of quantum state}

In the framework of quantum Hamilton mechanics, a wavefunction $\psi$ is not merely an abstract function in an infinite-dimensional vector space; it also represents a concrete dynamic system.

Definition 2.1. The quantum state assigned by a wavefunction $\psi$ is a dynamic system whose phase-space trajectory $(\mathbf{q}(t), \mathbf{p}(t))$ obeys the Hamilton equations (1.9) with the Hamiltonian $H$ given by Eq. (1.6).

According to this definition, when we say that a quantum observable $A(\mathbf{q}, \mathbf{p})$ is evaluated in the state $\psi_{0}$, it actually means that $A(\mathbf{q}, \mathbf{p})$ is evaluated along a phase-space trajectory $(\mathbf{q}(t), \mathbf{p}(t))$ determined from Eq. (1.9) with $H$ specified by $\psi_{0}$. On solving $(\mathbf{q}(t), \mathbf{p}(t))$ from Eq. (1.9), it can be shown as in the following theorem that the solution for $\mathbf{p}$ is already given by Eq. (1.8); that is to say, the solution of Schrödinger equation, $\psi$, provides the first integration of the Hamilton equations (1.9).

Theorem 2.1. For a given solution $\psi$ of Schrödinger equation, the quantum momentum $\mathbf{p}$ determined from Eq. (1.8) and the quantum potential $Q(\psi(\mathbf{q}))$ determined from $E q .(1.7)$, satisfy automatically the quantum Newton equation (1.9b), i.e.,

$$
\dot{\mathbf{p}}=-\frac{\partial H}{\partial \mathbf{q}}=-\frac{\partial}{\partial \mathbf{q}}(V+Q) .
$$

Proof. We shall start with Eq. (1.5) and show that it leads directly to Eq. (1.9b). The total differentiation of Eq. (1.5) with respect to the Cartesian coordinates $\mathbf{q}=\left(q_{1}, q_{2}, \ldots, q_{N}\right)$ reads 


$$
\frac{\mathrm{d}}{\mathrm{d} q_{i}}\left(\frac{\partial S}{\partial t}+H\right)=0, \quad i=1,2, \ldots, N,
$$

where with $S=S(t, \mathbf{q})$, we have $\mathrm{d}(\partial S / \partial t) / \mathrm{d} q_{i}=\partial^{2} S / \partial q_{i} \partial t$; while with $H=H(t, \mathbf{q}, \mathbf{p}(\mathbf{q}))$, we have

$$
\frac{\mathrm{d} H}{\mathrm{~d} q_{i}}=\frac{\partial H}{\partial q_{i}}+\sum_{k=1}^{N} \frac{\partial H}{\partial p_{k}} \frac{\partial p_{k}}{\partial q_{i}}
$$

with $p_{k}$ given by Eq. (1.8) as $p_{k}=\partial S / \partial q_{k}$. Assuming that $S$ is twice continuously differentiable with respect to $q_{k}$, we may rewrite $\partial p_{k} / \partial q_{i}$ as

$$
\frac{\partial p_{k}}{\partial q_{i}}=\frac{\partial}{\partial q_{i}} \frac{\partial S}{\partial q_{k}}=\frac{\partial}{\partial q_{k}} \frac{\partial S}{\partial q_{i}}=\frac{\partial p_{i}}{\partial q_{k}} .
$$

Inserting the above identity and Eq. (1.9a) into Eq. (2.3) yields

$$
\frac{\mathrm{d} H}{\mathrm{~d} q_{i}}=\frac{\partial H}{\partial q_{i}}+\sum_{k=1}^{N} \frac{\partial p_{i}}{\partial q_{k}} \dot{q}_{k},
$$

from which Eq. (2.2) becomes

$$
\frac{\partial p_{i}}{\partial t}+\sum_{k=1}^{N} \frac{\partial p_{i}}{\partial q_{k}} \dot{q}_{k}=-\frac{\partial H}{\partial q_{i}}
$$

This is just Eq. (1.9b) by noting that the left-hand side is equal to $\mathrm{d} p_{i} / \mathrm{d} t$, the total differentiation of $p_{i}$ with respect to time $t$.

The inverse of Theorem 2.1 is also true, i.e., starting with Hamilton equations (1.9) and assuming the solution of $p$ in the form of Eq. (1.8), we can show that the to-be-determined functions $S$ and $\psi$ satisfy the quantum $\mathrm{H}-\mathrm{J}$ equation (1.5) and the Schrödinger equation (1.2), respectively.

Example 2.1. As an illustrating example of Theorem 2.1, we consider the dynamic systems corresponding to the quantum states of harmonic oscillator. The Hamiltonian (1.6) with $V=K x^{2} / 2$ has the form

$$
H\left(\psi_{n}\right)=\frac{1}{2 m} p^{2}+\frac{1}{2} K x^{2}-\frac{\hbar^{2}}{2 m} \frac{\mathrm{d}^{2}}{\mathrm{~d} x^{2}} \ln \psi_{n}(x) .
$$

The eigenfunction $\psi_{n}(x)$ for harmonic oscillator is found to be

$$
\psi_{n}(x)=C_{n} H_{n}(\alpha x) \mathrm{e}^{-\alpha x^{2} / 2}, \quad n=0,1,2, \ldots
$$

where $\alpha=\sqrt{m k} / \hbar$ and $H_{n}$ is the $n$ th-order Hermite polynomial. The dynamic system associated with $\psi_{n}(x)$ is represented by the following Hamilton equations:

$$
\begin{aligned}
& \frac{\mathrm{d} x}{\mathrm{~d} t}=\frac{\partial H\left(\psi_{n}\right)}{\partial p}=\frac{p}{m} \\
& \frac{\mathrm{d} p}{\mathrm{~d} t}=-\frac{\partial H\left(\psi_{n}\right)}{\partial x}=-\frac{\mathrm{d}}{\mathrm{d} x}\left(\frac{1}{2} K x^{2}-\frac{\hbar^{2}}{2 m} \frac{\mathrm{d}^{2}}{\mathrm{~d} x^{2}} \ln \psi_{n}(x)\right)=-\frac{\mathrm{d}}{\mathrm{d} x}(V+Q) .
\end{aligned}
$$


Substituting Eq. (2.7a) into Eq. (2.7b) yields,

$$
m \frac{\mathrm{d} x^{2}}{\mathrm{~d} t^{2}}=-\frac{\mathrm{d} V}{\mathrm{~d} x}-\frac{\mathrm{d} Q}{\mathrm{~d} x} .
$$

This equation has the form of Newton's second law, in which the particle is subjected to a quantum force $-\mathrm{d} Q / \mathrm{d} x$ in addition to the classical force $-\mathrm{d} V / \mathrm{d} x$. Treating $p$ as a function of $x$, we can recast $\mathrm{d} p / \mathrm{d} t$ into the form

$$
\frac{\mathrm{d} p}{\mathrm{~d} t}=\frac{\mathrm{d} p}{\mathrm{~d} x} \frac{\mathrm{d} x}{\mathrm{~d} t}=\frac{p}{m} \frac{\mathrm{d} p}{\mathrm{~d} x}=\frac{1}{2 m} \frac{\mathrm{d}}{\mathrm{d} x} p^{2} .
$$

Combination of Eqs. (2.7b) and (2.9) yields

$$
H(x, p)=\frac{1}{2 m} p^{2}+V(x)+Q=E=\text { constant }
$$

where $Q$ is the quantum potential present in the state $\psi_{n}$

$$
Q=-\frac{\hbar^{2}}{2 m} \frac{\mathrm{d}^{2}}{\mathrm{~d} x^{2}} \ln \psi_{n}(x) .
$$

Accordingly, we can regard Eq. (2.10) as the energy conservation law for one-dimensional quantum Hamilton system. By substituting the relation

$$
p=-\mathrm{i} \hbar \frac{\mathrm{d}}{\mathrm{d} x} \ln \psi_{n}(x)=-\frac{\mathrm{i} \hbar}{\psi_{n}} \frac{\mathrm{d} \psi_{n}(x)}{\mathrm{d} x}
$$

from Eq. (1.8) into Eq. (2.10), it turns out that Eq. (2.10) is just the time-independent Schrödinger equation for harmonic oscillator

$$
\frac{\hbar^{2}}{2 m} \frac{\mathrm{d}^{2} \psi_{n}}{\mathrm{~d} x^{2}}+(E-V(x)) \psi_{n}=0 .
$$

The equivalence among the Hamilton equations (2.7), the energy conservation law (2.10) and the Schrödinger equation (2.13) indicates that the wavefunction $\psi_{n}$ actually represents a dynamic system whose behavior obeys the Hamilton equations (2.7), as stated in Definition 2.1 .

The constant $C_{n}$ in Eq. (2.6) has an important role in normalizing the wavefunction $\psi_{n}$, but as can be seen from Eq. (2.12), the dynamic representation of $\psi_{n}$ is independent of $C_{n}$. An alternative expression of Eq. (2.10) may be obtained by replacing $\psi_{n}$ with $p$ via the relation (2.12),

$$
H(x, p)=\frac{1}{2 m} p^{2}+V(x)+\frac{1}{2 m} \frac{\hbar}{\mathrm{i}} \frac{\mathrm{d} p}{\mathrm{~d} x}=E,
$$

which was known as Riccati equation in the mathematical literature. Quantum HamiltonJacobi theory [2,3], which was developed from Eq. (2.14), permits the exact determination of the bound-state energy levels and the related eigenfunctions without the necessity of solving the corresponding Schrödinger equation.

The quantum potential $Q$ has a close relation to the probability interpretation of standard quantum mechanics; it explains the underlying reason why some locations are hard to access, while some are accessible with large probability. 
Theorem 2.2. The total potential $V_{\text {Total }}=Q+V$ is inversely proportional to the probability density function $\psi^{*} \psi$ in the manner that the locations with zero probability are where the total potential approaches infinity and the locations with maximum probability are where the total potential arrives at its minimum. Moreover, the locations with maximum probability are just the equilibrium points of the dynamic system (1.9a) and (1.9b) representing the quantum state $\psi$.

Proof. From the energy conservation law of Eq. (2.10), we can express the total potential as

$$
V_{\text {Total }}=Q+V=E-\frac{1}{2 m} p^{2}=E+\frac{\hbar^{2}}{2 m}\left(\frac{\mathrm{d} \ln \psi}{\mathrm{d} x}\right)^{2} .
$$

A succinct expression of quantum Newton equation (2.8) then turns out to be

$$
m \ddot{x}=-\frac{\mathrm{d} V_{\text {Total }}}{\mathrm{d} x}=-\frac{\hbar^{2}}{2 m} \frac{\mathrm{d}}{\mathrm{d} x}\left(\frac{\mathrm{d} \ln \psi}{\mathrm{d} x}\right)^{2} .
$$

Since this equation of motion is independent of the constant $E$, we can choose $E=0$ as the reference energy level for $V_{\text {Total }}$. The magnitude of the total potential barrier now becomes

$$
\left|V_{\text {Total }}\right|=\left|\frac{\hbar^{2}}{2 m}\left(\frac{\mathrm{d} \ln \psi}{\mathrm{d} x}\right)^{2}\right|=\frac{\hbar^{2}}{2 m} \frac{|\mathrm{d} \psi / \mathrm{d} x|^{2}}{\psi^{*} \psi},
$$
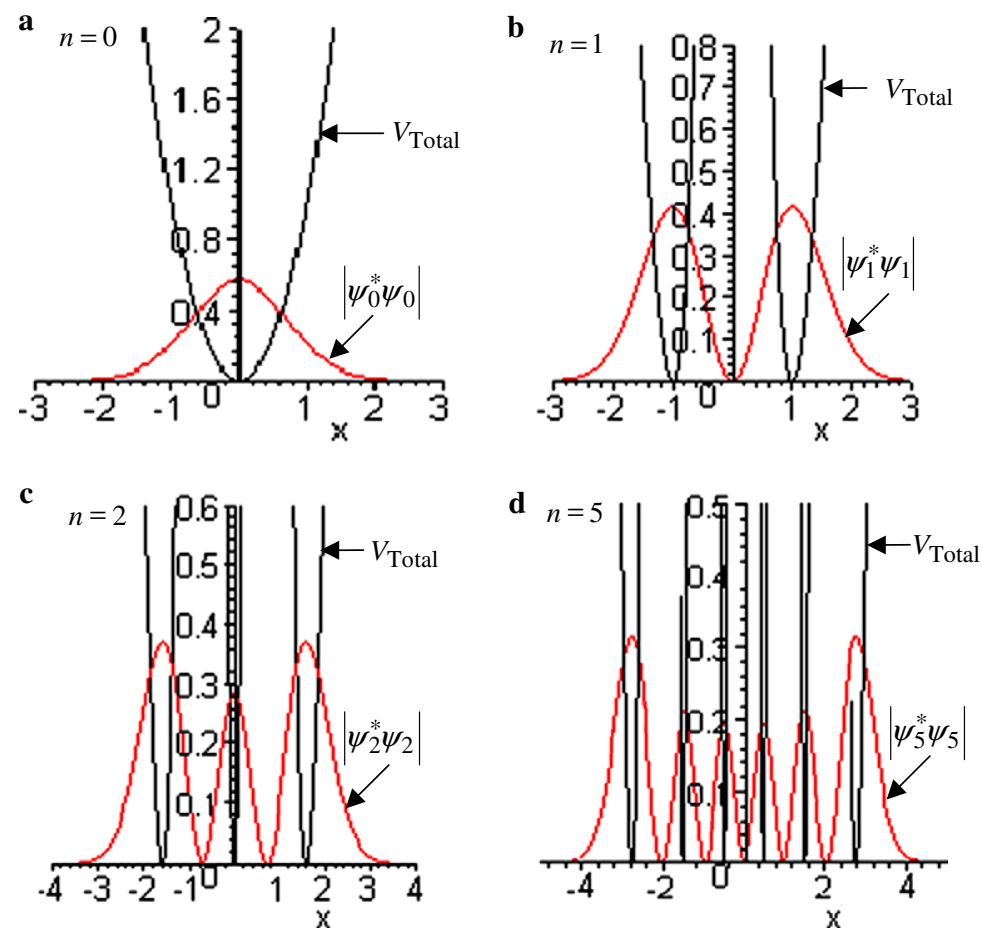

Fig. 1. The illustration of the inverse proportionality between the probability density $\left|\psi^{*} \psi\right|$ and the total potential $V_{\text {Total }}=V+Q(\psi)$ for harmonic oscillator with quantum states $n=0,1,2$, and 5 . The locations with zero probability are where the total potential approaches infinity and the locations with maximum probability are where the total potential arrives at its minimum. (a) $n=0$. (b) $n=1$. (c) $n=2$. (d) $n=5$. 
which states that the height of the total potential barrier is inversely proportional to $\psi^{*} \psi$. A spatial point with large value of $\psi^{*} \psi$ corresponds to the location of low potential barrier and hence large accessibility to this point. This fact legitimates the use of $\psi^{*} \psi$ as the probability measure for a particle to appear at a specified spatial point. Besides the probability information provided by $\psi^{*} \psi$, the detailed trajectory under the action of $V_{\text {Total }}$ can be found by integrating Eq. (2.16) whose first integration, as has been shown in Theorem 2.1 , is given by Eqs. (1.8) and (1.9a)

$$
\frac{\mathrm{d} x}{\mathrm{~d} t}=\frac{p}{m}=-\frac{\mathrm{i} \hbar}{m} \frac{\mathrm{d} \psi / \mathrm{d} x}{\psi} .
$$

The equilibrium point of the above nonlinear system is the position having the property of $\dot{x}=0$, i.e., $\mathrm{d} \psi / \mathrm{d} x=0$, which in turn is the necessary condition that the probability density $\psi^{*} \psi$ achieves its maximum.

Fig. 1 is an illustration of Theorem 2.2 by taking harmonic oscillator as an example. The total potential $V_{\text {Total }}$ and the probability density $\psi^{*} \psi$ are plotted together for quantum states $n=0,1,2$, and 5 . The inverse proportionality between $V_{\text {Total }}$ and $\psi^{*} \psi$ is clearly displayed, from which the positions with zero probability are justified by the presence of infinity potential and the positions with the maximum probability are justified by the lowermost points of the potential.

\section{Relating quantum operator to complex observable}

Besides the state-dependent property, quantum Hamiltonian mechanics is distinct from classical Hamiltonian mechanics in the unique feature that the observables appeared in quantum Hamilton mechanics, such as $\mathbf{q}, \mathbf{p}$, and $H$, are in general complex-valued. For instance, if we determine the momentum $\mathbf{p}$ from Eq. (1.8) for a given wavefunction $\psi$, we should find that $\mathbf{p}$ has real component as well as imaginary component. This complex-valued nature inherits from Schrödinger equation, which produces the complex-valued wavefunction that, in turn, leads to the complex-valued quantum Hamiltonian $H$ and quantum potential $Q$. It is this complex-valued nature that allows us to derive the correct quantum operator accompanying each quantum observable. In the following, we first introduce the definition of quantum operator in Hamilton mechanics, and then demonstrate how the commonly used quantum operators in Cartesian coordinates can be derived from this definition. Operators in curvilinear coordinates will be considered in the later sections.

Definition 3.1. For a quantum observable $A$ evaluated in the quantum state $\psi$, its associated quantum operator $\hat{A}$ is defined via the relation

$$
A=\frac{1}{\psi} \hat{A} \psi
$$

Using this definition, we can give a formal proof of the quantization axiom $\mathbf{p} \rightarrow-\mathrm{i} \hbar \nabla$ that governs the critical transition from classical systems to quantum-mechanical systems.

Theorem 3.1. The canonical operators $(\hat{\mathbf{q}}, \hat{\mathbf{p}})$ corresponding to the canonical variables $(\mathbf{q}, \mathbf{p})$ are given by $\hat{\mathbf{q}}=\mathbf{q}$ and $\hat{\mathbf{p}}=-\mathrm{i} \hbar \nabla$, whereas the Hamiltonian operator $\hat{H}$ corresponding to the quantum Hamiltonian defined in Eq. (1.6) is given by $\hat{H}=-\hat{\mathbf{p}}^{2} / 2 m+V$. 
Proof. Rewriting Eq. (1.8) in the form of Eq. (3.1), we obtain

$$
\mathbf{p}=\nabla S=-\mathrm{i} \hbar \nabla \ln \psi=(1 / \psi)(-\mathrm{i} \hbar \nabla) \psi .
$$

The comparison of the above equation with the definition $\mathbf{p}=(1 / \psi) \hat{\mathbf{p}} \psi$ gives $\hat{\mathbf{p}}=-\mathrm{i} \hbar \nabla$. As for $\hat{\mathbf{q}}$, we may express $\mathbf{q}$ as $\mathbf{q}=(1 / \psi) \mathbf{q} \psi$ and contrast this with the definition $\mathbf{q}=(1 / \psi) \hat{\mathbf{q}} \psi$ to obtain $\hat{\mathbf{q}}=\mathbf{q}$. To derive the Hamiltonian operator $\hat{H}$ from the definition $H=(1 / \psi) \hat{H} \psi$, we need to express the quantum Hamiltonian $H$ in terms of the wavefunction $\psi$. The insertion of $\mathbf{p}=-\mathrm{i} \hbar \nabla \ln \psi$ in Eq. (1.6) yields

$$
H=\frac{1}{2 m}(-\mathrm{i} \hbar \nabla \ln \psi)^{2}-\frac{\hbar^{2}}{2 m} \nabla^{2} \ln \psi+V=\frac{1}{\psi}\left(\frac{-\hbar^{2}}{2 m} \nabla^{2}+V\right) \psi .
$$

In comparison with Eq. (3.1), Eq. (3.3) produces the Hamiltonian operator $\hat{H}=$ $-\hat{\mathbf{p}}^{2} / 2 m+V$.

The canonical momentum operator $\hat{\mathbf{p}}$ derived in Theorem 3.1 must not be confused with the mechanical momentum operator $\hat{\mathbf{P}}$. In Cartesian coordinates, the mechanical momentum $\mathbf{P}$ is given by $\mathbf{P}=m \dot{\mathbf{q}}$ and from Eq. (1.9a) we find $\mathbf{p}=m \dot{\mathbf{q}}=\mathbf{P}$, which indicates $\hat{\mathbf{P}}=\hat{\mathbf{p}}=-\mathrm{i} \hbar \nabla$ in Cartesian coordinates. However, in curvilinear coordinates, $\mathbf{p}$ and $\mathbf{P}$ are, in general, different and the quantization axiom $\hat{\mathbf{P}}=-\mathrm{i} \hbar \nabla$ is no longer valid as will be expounded further in Theorem 7.2.

Eq. (3.2) indicates that defining quantum momentum $\mathbf{p}=\nabla S$ in complex domain is necessary to result in the correct momentum operator $\hat{\mathbf{p}}$. A similar but different quantum momentum was proposed by Bohm [4] in the form of $\mathbf{p}_{\mathrm{B}}=\nabla S_{\mathrm{B}}$, where $S_{\mathrm{B}}$ is the phase of the wavefunction defined by $\psi=R_{\mathrm{B}} \mathrm{e}^{\mathrm{i} S_{\mathrm{B}} / \hbar}$ with $R_{\mathrm{B}}$ and $S_{\mathrm{B}}$ being real functions. If we follow the same procedures leading to Eq. (3.2) but employ the real quantum momentum $\mathbf{p}_{\mathrm{B}}=\nabla S_{\mathrm{B}}$ instead of the complex momentum $\mathbf{p}=\nabla S$, we shall find that it is not possible to arrive at the correct momentum operator $\hat{\mathbf{p}}=-\mathrm{i} \hbar \nabla$.

A natural outcome of defining canonical variables $(\mathbf{q}, \mathbf{p})$ in complex domain is the quantization of action variable, a postulate proposed by Sommerfeld and Wilson,

$$
J_{i}=\oint_{c_{i}} p_{i} \mathrm{~d} q_{i}=n h, \quad n=0,1,2, \cdots, \quad i=1,2, \cdots, N,
$$

where $c_{i}$ is a closed trajectory in the complex $q_{i}$ plane obtained from the integration of the Hamilton equations (1.9) and the complex momentum $p_{i}$ is given by Eq. (1.8) as $p_{i}=-\mathrm{i} \hbar \partial \ln \psi / \partial q_{i}$. To prove the quantization rule (3.4), we define the following conformal mappings from the $q_{i}$ complex plane to the $\psi$ complex plane:

$$
\psi_{i}=\psi\left(q_{i}\right): q_{i} \rightarrow \psi, \quad i=1,2, \ldots, N,
$$

where $\psi\left(q_{i}\right)$ is a function of the single complex variable $q_{i}$ obtained by fixing other coordinates in the wavefunction $\psi\left(q_{1}, \ldots, q_{i}, \ldots, q_{N}\right)$. The function $\psi_{i}$ maps a closed path $c_{i}$ in the $q_{i}$ plane into a closed path $c_{i}^{\prime}$ in the $\psi$ plane. Counting the number of encirclement of the origin in the $\psi$ plane by the closed path $c_{i}^{\prime}$ provides us with the quantum number $n$ in Eq. (3.4).

Theorem 3.2. Let $c_{i}$ be any closed complex trajectory traced out by the coordinate $q_{i}$. Then the contour integral defined in Eq. (3.4) over the contour $c_{i}$ is quantized and the related quantum number $n$ is equal to the number of encirclement of the origin in the $\psi$ plane by the closed path $c_{i}^{\prime}$ obtained from $c_{i}$ via the mapping $\psi_{i}$. 


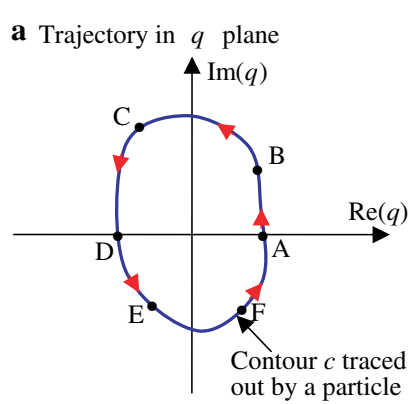

c One encirclement of the origin

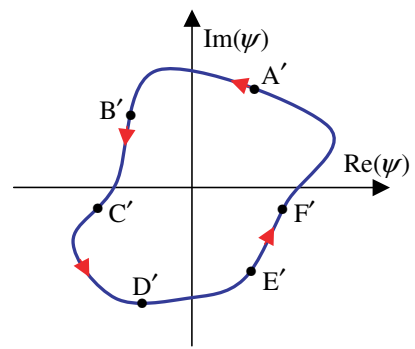

b Zero encirclement of the origin

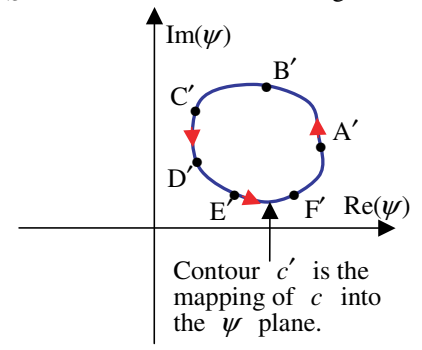

d Two encirclements of the origin

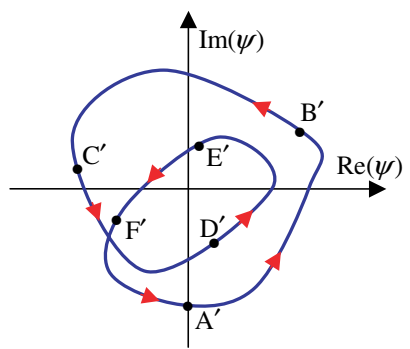

Fig. 2. Conformal mapping of the closed contour $c$ in the $q$ plane into the closed contour $c^{\prime}$ in the $\psi$ plane. (a) Trajectory in $q$ plane. (b) Zero encirclement of the origin. (c) One encirclement of the origin. (d) Two encirclements of the origin. The contour $c$ is the particle's trajectory solved from quantum Hamilton equations of motion and the imagine contour $c^{\prime}$ is obtained via the transformation $\psi=\psi(q)$. As a representative point $q(t)$ traces out the entire contour $c$ in the counterclockwise direction $A \rightarrow B \rightarrow C \rightarrow D \rightarrow E \rightarrow F$, its image point in the $\psi$ plane traces out the contour $c^{\prime}$ in the direction $A^{\prime} \rightarrow B^{\prime} \rightarrow C^{\prime} \rightarrow D^{\prime} \rightarrow E^{\prime} \rightarrow F^{\prime}$. The number $n$ of the counterclockwise encirclements of the origin of the $\psi$ plane is equal to 0,1 , and 2 , respectively, for the case (b), (c), and (d).

Proof. With the substitution $p_{i}=-\mathrm{i} \hbar \partial \ln \psi / \partial q_{i}$, the action variable $J_{i}$ becomes

$$
J_{i}=\oint_{c_{i}} p_{i} \mathrm{~d} q_{i}=\frac{\hbar}{\mathrm{i}} \oint_{c_{i}} \frac{\partial \ln \psi}{\partial q_{i}} \mathrm{~d} q_{i}=\frac{\hbar}{\mathrm{i}} \oint_{c_{i}} \frac{d \ln \psi_{i}}{d q_{i}} \mathrm{~d} q_{i}=\frac{\hbar}{\mathrm{i}} \oint_{c_{i}^{\prime}} \mathrm{d} \ln \psi_{i},
$$

where in the last equality we have expressed $J_{i}$ in terms of the net change of $\ln \psi_{i}$ along the closed path $c_{i}^{\prime}$. By expressing $\psi_{i}$ in a polar form $\psi_{i}=\left|\psi_{i}\right| \mathrm{e}^{\mathrm{i} \theta}$, we can further simplify $J_{i}$ as

$$
J_{i}=\frac{\hbar}{\mathrm{i}} \oint_{c_{i}^{\prime}} \mathrm{d} \ln \left|\psi_{i}\right|+\hbar \oint_{c_{i}^{\prime}} \mathrm{d} \theta
$$

The first term in the right-hand side is zero because the net change of $\ln \left|\psi_{i}\right|$ is zero along any closed path. The second term is relevant to the net phase change of $\psi_{i}$ along the closed path $c_{i}^{\prime}$, which must be an integral multiple of $2 \pi$. Therefore, Eq. (3.7) is reduced to the expected result

$$
J_{i}=\hbar \oint_{c_{i}^{\prime}} \mathrm{d} \theta=\hbar(2 n \pi)=n h,
$$

where $n$ is the number of encirclement of the origin in the $\psi_{i}$ plane by the closed path $c_{i}^{\prime}$ and $2 n \pi$ represents the corresponding net phase change. Fig. 2 illustrates the mapping between 
the contour $c_{i}$ in the complex plane $q_{i}$ and the contour $c_{i}^{\prime}$ in the complex $\psi_{i}$ plane. Observing the number of encirclement of the origin by the closed path $c_{i}^{\prime}$ allows us to identify the quantum number $n$ graphically.

The quantization rule (3.4) is independently valid for any coordinate $q_{i}(t)$ that has periodic motion and thus has closed trajectory in the complex $q_{i}$ plane, regardless of whether the whole quantum system is periodic or not. We shall revisit Theorem 3.2 in Section 4 for the quantization of harmonic oscillator and in Section 6 for the quantization of hydrogen atom. In Theorems 3.1 and 3.2, we have witnessed the necessity of extending quantum observables such as $\mathbf{q}$ and $\mathbf{p}$ to complex domain. It is worth noting that the relation between the complex quantum observable $A$ and the quantum operator $\hat{A}$ in Eq. (3.1) is in the form of strict equality, but not merely an abstract correspondence. In the standard approach, we obtained $\hat{H}$ by applying the abstract corresponding principle of replacing the momentum $\mathbf{p}$ in the classical Hamiltonian $H_{\mathrm{c}}=\mathbf{p}^{2} / 2 m+V$ with the momentum operator $\hat{\mathbf{p}}$, but in doing so we could not establish any equality between $\hat{H}$ and $H_{\mathrm{c}}$. However, under the framework of quantum Hamiltonian mechanics, we have a quantum Hamiltonian $H$ in Eq. (1.6), which is directly related to $\hat{H}$ via the equality $H=(1 / \psi) \hat{H} \psi$.

For any given observable $A$ defined in quantum Hamilton mechanics, we can identify its accompanying operator $\hat{A}$ by using Eq. (3.1); conversely, for a given operator $\hat{B}$, an explicit expression for its accompanying quantum observable $B$ is found to be $B=$ $(1 / \psi) \hat{B} \psi$. To familiarize us with this equivalence, let us consider a textbook example regarding the operator of the angular momentum $\mathbf{L}=\mathbf{q} \times \mathbf{p}$.

Example 3.1. In quantum Hamilton mechanics, the expression for $\mathbf{L}$ is the same but with $\mathbf{q}=\left[\begin{array}{lll}x & y & z\end{array}\right]$ and $\mathbf{p}=\left[p_{x} p_{y} p_{z}\right]$ satisfying the quantum Hamilton equations (1.9), instead of the classical Hamilton equations. Evaluating the $x$ components of $\mathbf{L}$ with $\mathbf{p}$ given by Eq. (1.8), we obtain

$$
L_{x}=y p_{z}-z p_{y}=y\left(\frac{\hbar}{\mathrm{i}} \frac{\partial \ln \psi}{\partial z}\right)-z\left(\frac{\hbar}{\mathrm{i}} \frac{\partial \ln \psi}{\partial y}\right)=\frac{-\mathrm{i} \hbar}{\psi}\left(y \frac{\partial}{\partial z}-z \frac{\partial}{\partial y}\right) \psi .
$$

Comparing the above equation to the definition $L_{x}=(1 / \psi) \hat{L}_{x} \psi$ gives $\hat{L}_{x}$ as:

$$
\hat{L}_{x}=-\mathrm{i} \hbar\left(y \frac{\partial}{\partial z}-z \frac{\partial}{\partial y}\right)=y \hat{p}_{z}-z \hat{p}_{y}
$$

where the expressions for $\hat{p}_{y}$ and $\hat{p}_{z}$ have been derived in Theorem 3.1. We recognize that an explicit expression of $L_{x}$ in terms of the wavefunction $\psi$ naturally leads to the expression for $\hat{L}_{x}$. The other two components $\hat{L}_{y}=z \hat{p}_{x}-x \hat{p}_{z}$ and $\hat{L}_{z}=x \hat{p}_{y}-y \hat{p}_{x}$ can be derived in a similar way.

There exists a special wavefunction $\psi_{n}$ such that Eq. (3.1) yields $A(\mathbf{p}, \mathbf{q})=A_{n}=$ $\left(1 / \psi_{n}\right) \hat{A} \psi_{n}=$ constant. In such a case, the observable $A(\mathbf{p}, \mathbf{q})$ becomes a constant in the state $\psi_{n}$. In conjunction with $\psi_{n}$, a remarkable link can be established between the conservation law in quantum Hamilton mechanics and the concept of stationary observable in quantum mechanics.

Lemma 3.1. A quantum observable $A(\mathbf{p}, \mathbf{q})$ is stationary in the quantum state $\psi_{n}$, if and only if $A(\mathbf{p}, \mathbf{q})$ is conservative along any phase-space trajectory $(\mathbf{q}(t), \mathbf{p}(t))$ determined from Eq. (1.9) with the Hamiltonian $H$ induced by $\psi_{n}$; furthermore, this conserved value of $A(\mathbf{p}, \mathbf{q})$ is just equal to the eigenvalue of $\hat{A}$ with respect to the eigenfunction $\psi_{n}$. 
Proof. In quantum mechanics, an observable $A$ is said to be stationary in the state $\psi_{n}$, if its related operator $\hat{A}$ satisfies $\hat{A} \psi_{n}=A_{n} \psi_{n}$, where $A_{n}$ is the eigenvalue of $\hat{A}$ corresponding to the eigenfunction $\psi_{n}$. Now we can apply Eq. (3.1) to evaluate $A$ in the state $\psi_{n}$ as $A(\mathbf{q}, \mathbf{p})=\left(1 / \psi_{n}\right) \hat{A} \psi_{n}=\left(1 / \psi_{n}\right) A_{n} \psi_{n}=A_{n}$, which states that the value of $A(\mathbf{p}, \mathbf{q})$ evaluated in the state $\psi_{n}$ is a constant equal to the eigenvalue $A_{n}$. According to Definition 3.1 , the constancy of $A(\mathbf{q}, \mathbf{p})$ in the state $\psi_{n}$ amounts to the conservation of $A(\mathbf{p}, \mathbf{q})$ along any phase-space trajectory $(\mathbf{q}(t), \mathbf{p}(t))$ determined from Eq. (1.9) with the Hamiltonian $H$ induced by $\psi_{n}$. Conversely, if we are given that $A$ is a constant $A_{n}$ in the state $\psi_{n}$, then Eq. (3.1) implies $A=A_{n}=\left(1 / \psi_{n}\right) \hat{A} \psi_{n}$, i.e., $\hat{A} \psi_{n}=A_{n} \psi_{n}$, which ensures that $A$ is stationary in $\psi_{n}$.

As a demonstration of Lemma 3.1, we consider $\hat{A}=\hat{H}$ and assume that $H$ is stationary in $\psi_{n}$, i.e., $\hat{H} \psi_{n}=E_{n} \psi_{n}$. If we apply this stationary condition to Eq. (3.1), we obtain $H(\mathbf{q}, \mathbf{p})=\left(1 / \psi_{n}\right) \hat{H} \psi_{n}=\left(1 / \psi_{n}\right) E_{n} \psi_{n}=E_{n}$, showing that the Hamiltonian $H(\mathbf{q}(t), \mathbf{p}(t))$ in Eq. (1.6) is conservative along any phase-space trajectory $(\mathbf{q}(t), \mathbf{p}(t))$ in the state $\psi_{n}$. We may confirm the conservation of $H$ by showing $\mathrm{d} H / \mathrm{d} t=0$ in the state $\psi_{n}$. Because $\mathbf{q}(t)$ and $\mathbf{p}(t)$ in the state $\psi_{n}$ satisfy the Hamilton equations (1.9), we obtain the expected result

$$
\frac{\mathrm{d} H}{\mathrm{~d} t}=\frac{\partial H}{\partial \mathbf{q}} \frac{\mathrm{d} \mathbf{q}}{\mathrm{d} t}+\frac{\partial H}{\partial \mathbf{p}} \frac{\mathrm{d} \mathbf{p}}{\mathrm{d} t}=\frac{\partial H}{\partial \mathbf{q}} \frac{\partial H}{\partial \mathbf{p}}-\frac{\partial H}{\partial \mathbf{p}} \frac{\partial H}{\partial \mathbf{q}}=0 .
$$

Example 3.2. Continue the discussion of harmonic oscillator in Example 2.1 and consider the energy conservation in the ground state, for which the wavefunction is given by $\psi_{0}=C_{0} H_{0} \mathrm{e}^{-\alpha x^{2}}$. Inserting $\psi_{0}$ into Eqs. (2.11) and (2.12) yields, respectively, the quantum potential $Q=\hbar \omega / 2=(\hbar / 2) \sqrt{K / m}$ and the quantum momentum $p=\mathrm{i} \hbar \alpha x$. The total energy in the ground state is then found to be a constant equal to

$$
H=\frac{p^{2}}{2 m}+\frac{1}{2} K x^{2}+Q=-\frac{1}{2 m} \hbar^{2} \alpha^{2} x^{2}+\frac{1}{2} K x^{2}+\frac{\hbar \omega}{2}=\frac{\hbar \omega}{2}=E_{0},
$$

where $E_{0}=\hbar \omega / 2$ is just the eigenvalue corresponding to $\psi_{0}$. By a similar way, we can show in the $n$th eigenstate $\psi_{n}$, the total energy $H$ is a constant equal to the eigenvalue corresponding to $\psi_{n}$, i.e., $H=(n+1 / 2) \hbar \omega=E_{n}$.

On the other hand, when $\psi$ is not an eigenfunction of $\hat{A}$, we have $A(\mathbf{q}, \mathbf{p})=$ $(1 / \psi) \hat{A} \psi \neq$ constant with its value being varying with $(\mathbf{q}(t), \mathbf{p}(t))$. In such a case, quantum mechanics says that $A$ is uncertain in the state $\psi$ and suggests adopting $\psi^{*} \psi$ as the probability density function to extract the statistical properties of $A$. Quantum Hamilton mechanics provides us with an alternative way to evaluate a nonstationary observable; we may employ the expression $A(\mathbf{q}, \mathbf{p})=(1 / \psi) \hat{A} \psi$ to explicitly trace the variation of $A(\mathbf{q}(t), \mathbf{p}(t))$ along any phase-space trajectory $(\mathbf{q}(t), \mathbf{p}(t))$ in the dynamic system (1.9) prescribed by $\psi$.

The equivalence established in Lemma 3.1 can be elucidated more concisely in terms of the equality (not merely a correspondence) between the commutator defined in quantum mechanics and the Poisson bracket defined in Hamilton mechanics. Given two operators $\hat{A}$ and $\hat{B}$, their commutator is defined as $[\hat{A}, \hat{B}]=\hat{A} \hat{B}-\hat{B} \hat{A}$. The accompanying observables $A$ and $B$ evaluated in the state $\psi$ are given, respectively, as $A(\mathbf{q}, \mathbf{p})=(1 / \psi) \hat{A} \psi$ and 
$\hat{B}(\mathbf{q}, \mathbf{p})=(1 / \psi) \hat{B} \psi$ according to Definition 3.1. Having obtained $A(\mathbf{q}, \mathbf{p})$ and $B(\mathbf{q}, \mathbf{p})$, we can evaluate the Poisson bracket of $A$ and $B$ in a usual way:

$$
\{A, B\}=\sum_{i} \frac{\partial A}{\partial q_{i}} \frac{\partial B}{\partial p_{i}}-\frac{\partial A}{\partial p_{i}} \frac{\partial B}{\partial q_{i}} .
$$

Lemma 3.2. If an observable $A(\mathbf{q}, \mathbf{p})$ is stationary in the eigenfunction $\psi$ of $\hat{H}$, then

$$
\{A, H\}=\frac{1}{\psi}[\hat{A}, \hat{H}] \psi=0 .
$$

Proof. For the given condition, we have $\hat{H} \psi=E_{n} \psi$ and $\hat{A} \psi=A_{n} \psi$, which yields $\hat{A} \hat{H} \psi=\hat{H} \hat{A} \psi=A_{n} E_{n} \psi$ and hence $[\hat{A}, \hat{H}] \psi=(\hat{A} \hat{H}-\hat{H} \hat{A}) \psi=0$. On the other hand, evaluating $A$ in the state $\psi$ results in $A(\mathbf{q}, \mathbf{p})=(1 / \psi) \hat{A} \psi=(1 / \psi) A_{n} \psi=A_{n}$. This means that $A(\mathbf{q}(t), \mathbf{p}(t))$ is conservative along any phase-space trajectory $(\mathbf{q}(t), \mathbf{p}(t))$ in the state $\psi$. From the viewpoint of Hamilton mechanics, the conservation of $A(\mathbf{q}(t), \mathbf{p}(t))$ requires

$$
\frac{\mathrm{d}}{\mathrm{d} t} A(\mathbf{q}(t), \mathbf{p}(t))=0=\sum_{i} \frac{\partial A}{\partial q_{i}} \dot{q}_{i}+\frac{\partial A}{\partial p_{i}} \dot{p}_{i}=\sum_{i} \frac{\partial A}{\partial q_{i}} \frac{\partial H}{\partial p_{i}}-\frac{\partial A}{\partial p_{i}} \frac{\partial H}{\partial q_{i}},
$$

where $\mathbf{q}(t)$ and $\mathbf{p}(t)$ satisfy the Hamilton equations (1.9) specified by $\psi$. The above equation amounts to $\{A, H\}=0$ which, together with $[\hat{A}, \hat{H}] \psi=0$, gives Eq. (3.13).

We say that $\hat{A}$ and $\hat{H}$ satisfying Eq. (3.13) are compatible with each other. For two operators $\hat{A}$ and $\hat{B}$ that are incompatible, their commutator and Poisson bracket are both nonzero, but useful relation between $\{A, B\}$ and $[\hat{A}, \hat{B}]$ still exists. One typical example comes from the case of $\hat{x}$ and $\hat{p}_{x}$, which have commutator $\left[\hat{x}, \hat{p}_{x}\right]=\mathrm{i} \hbar$ and Poisson bracket $\left\{x, p_{x}\right\}=1$. Expressing them in a form analogous to Eq. (3.13), we have

$$
\mathrm{i} \hbar\left\{x, p_{x}\right\}=\frac{1}{\psi}\left[\hat{x}, \hat{p}_{x}\right] \psi=\mathrm{i} \hbar .
$$

This suggests an identity that the operator corresponding to the observable $\mathrm{i} \hbar\{A, B\}$ is $[\hat{A}, \hat{B}]$, i.e.,

$$
\mathrm{i} \hbar\{A, B\}=\frac{1}{\psi}[\hat{A}, \hat{B}] \psi .
$$

This relation does hold for most commonly used quantum operators. The following examples cite two of them.

Example 3.3. Given the two observables $A=L_{x}=y p_{z}-z p_{x}$ and $B=L_{y}=z p_{x}-x p_{z}$, we have their Poisson bracket as $\left\{L_{x}, L_{y}\right\}=x p_{y}-y p_{x}=L_{z}$; on the other hand, the commutator of the associated operators $\hat{L}_{x}$ and $\hat{L}_{y}$ is known to be $\left[\hat{L}_{x}, \hat{L}_{y}\right]=\mathrm{i} \hbar \hat{L}_{z}$. Accordingly, we have

$$
\frac{1}{\psi}\left[\hat{L}_{x}, \hat{L}_{y}\right] \psi=\frac{1}{\psi}\left(\mathrm{i} \hbar \hat{L}_{z}\right) \psi=\mathrm{i} \hbar\left(\frac{1}{\psi} \hat{L}_{z} \psi\right)=\mathrm{i} \hbar L_{z}=\mathrm{i} \hbar\left\{L_{x}, L_{y}\right\},
$$

which satisfies the identity (3.15). 
Example 3.4. Consider $A=H$ and $B=x$, and their commutator $[\hat{H}, \hat{x}]=(-\mathrm{i} \hbar / m) \hat{p}_{x}$. The observable related to $[\hat{H}, \hat{x}]$ is found from the relation

$$
\frac{1}{\psi}[\hat{H}, \hat{x}] \psi=-\frac{\mathrm{i} \hbar}{m}\left(\frac{1}{\psi} \hat{p}_{x} \psi\right)=-\frac{\mathrm{i} \hbar}{m} p_{x} .
$$

This observable is to be linked to the Poisson bracket $\{H, x\}$ with $H$ given by Eq. (1.6),

$$
\{H, x\}=-\frac{\partial H}{\partial p_{x}} \frac{\partial x}{\partial x}=-\frac{1}{m} p_{x} .
$$

The combination of Eqs. (3.17) and (3.18) yields

$$
\mathrm{i} \hbar\{H, x\}=\frac{1}{\psi}[\hat{H}, \hat{x}] \psi,
$$

which again is a special case of Eq. (3.15).

\section{Harmonic oscillator in quantum Hamilton mechanics}

As mentioned in Definition 3.1, quantum Hamilton mechanics associates each quantum state $\psi$ with its equivalent dynamic representation (1.9). This representation not only permits us to derive quantum operators from their corresponding observables, as discussed in the last section, but also provides us with an alternative approach to extracting information from $\psi$. In this section, we shall demonstrate how to analyze quantum harmonic oscillator by the dynamic representation of $\psi$, instead of treating $\psi^{*} \psi$ as a probability density function.

Given the eigenfunction $\psi_{n}$ in Eq. (2.6) for harmonic oscillator, the related dynamic system is represented by Eq. (2.7). The solution for $p$ can be expressed in terms of $\psi_{n}$ as in Eq. (2.12) according to Theorem 2.1. Combining Eqs. (2.12) and (2.7a), we obtain

$$
\frac{\mathrm{d} x}{\mathrm{~d} t}=\frac{p}{m}=-\mathrm{i} \frac{\hbar}{m} \frac{1}{\psi_{n}} \frac{\mathrm{d} \psi_{n}(x)}{\mathrm{d} x},
$$

from which we can solve for the eigen-trajectory $x(t)$ in the eigenstate $\psi_{n}$. First, consider ground state $\psi_{0}=C_{0} \mathrm{e}^{-\alpha x^{2}}$ with $\alpha=\sqrt{m k} / \hbar$, for which Eq. (4.1) turns out to be $\dot{x}=\mathrm{i} \omega x$. The solution is found readily as

$$
x(t)=x_{\mathrm{R}}(t)+\mathrm{i} x_{\mathrm{I}}(t)=A \cos \omega t+\mathrm{i} A \sin \omega t,
$$

where $A$ is a constant determined from initial condition. It is worth noting that the realpart motion of the quantum harmonic oscillator in the ground state, which is described by $x_{\mathrm{R}}(t)=A \cos \omega t$, is identical to the solution of the classical harmonic oscillator $\ddot{x}_{\mathrm{R}}+\omega^{2} x_{\mathrm{R}}=0$. The other point to note is that the period of the circular motion described by Eq. (4.2) is $T=2 \pi / \omega=2 \pi \sqrt{m / k}$, the same as that of classical oscillator. This means that the quantum harmonic oscillator at the ground state has a period equal to that of the classical oscillator. The above results are consistent with the Feynman's prediction [5] about the dynamics of superconductivity, that at absolute zero temperature $(n=0)$ superconductivity is the same as the problem of the classical hydrodynamics of a charged liquid. The fact, that the zero-point dynamics at zero temperature can be described classically, can be easily verified by the equation of motion (2.7b). Substituting $\psi_{0}$ into Eq. (2.7b), we find 


$$
\text { quantum force }=-\frac{\mathrm{d} Q}{\mathrm{~d} x}=\frac{\hbar^{2}}{2 m} \frac{\mathrm{d}}{\mathrm{d} x}\left(\frac{\mathrm{d}^{2} \ln \psi_{0}}{\mathrm{~d} x^{2}}\right)=0 .
$$

Hence, zero-point dynamics are completely governed by the classical equation of motion:

$$
m \ddot{x}=-\frac{\mathrm{d} Q}{\mathrm{~d} x}-\frac{\mathrm{d} V}{\mathrm{~d} x}=-\frac{\mathrm{d} V}{\mathrm{~d} x}=-k x .
$$

The other remarkable property of zero-point dynamics is its energy content. Eq. (3.11) indicates that the classical energy $p^{2} /(2 m)+k x^{2} / 2$ is identically zero and the particle's energy is completely originated from the quantum potential $Q=\hbar \omega / 2$. Thus, the zero-point dynamics is solely due to quantum effect and independent of classical energy.

The eigen-trajectories in ground state comprise a set $\Omega_{\mathrm{c}}$ of concentric circles in complex plane described by Eq. (4.2). Along any one of these concentric circles, we have the following contour integral from Cauchy theorem as

$$
\oint_{c} p \mathrm{~d} x=i \hbar \alpha \oint_{c} x \mathrm{~d} x=0, \quad \forall c \in \Omega_{c},
$$

which justifies the Sommerfeld-Wilson quantization rules. The general form is of $\oint p \mathrm{~d} x=n h, n=0,1,2, \ldots$, and will be proved in the subsequent discussions.

Next, we consider the first excited state $\psi_{1}=2 \bar{x} \mathrm{e}^{-\bar{x}^{2} / 2}$ and apply it to Eq. (4.1) to yield

$$
\bar{p}=\frac{\mathrm{d} \bar{x}}{\mathrm{~d} \bar{t}}=\frac{-\mathrm{i}}{\psi_{1}(\bar{x})} \frac{\mathrm{d} \psi_{1}(\bar{x})}{\mathrm{d} \bar{x}}=\mathrm{i} \frac{\bar{x}^{2}-1}{\bar{x}}, \quad \bar{x}\left(\bar{t}_{0}\right)=\bar{x}_{0} \in \hbar,
$$

where we adopt the dimensionless variables as $\bar{x}=\sqrt{\alpha} x, \bar{p}=p /(\hbar \sqrt{\alpha}), \bar{t}=t \omega$, and $\bar{E}=$ $E /(\hbar \omega)$. The integration gives the eigen-trajectory in the $n=1$ state as

$$
\bar{x}(\bar{t})= \pm \sqrt{1+A \mathrm{e}^{2 \mathrm{i}}},
$$

where the constant $A$ is determined from the initial position $\bar{x}_{0}$. Several typical eigen-trajectories for $n=1$ are shown in Fig. 3a. The nonlinear dynamics governed by Eq. (4.6) have two equilibrium points $\bar{x}_{\mathrm{eq}}= \pm 1$. If a particle starts with $\bar{x}_{0}=\bar{x}_{\mathrm{eq}}$, it will continue to stay at $\bar{x}_{\text {eq }}$ since $\mathrm{d} \bar{x} / \mathrm{d} \bar{t}=0$ at this point. Bohm's prediction [4], that particle in any stationary state is motionless, is reasonable only if the particle locates at the equilibrium points of the stationary states. Since there is a tendency for the harmonic oscillator to recover to the equilibrium points, the equilibrium points $\bar{x}_{\mathrm{eq}}= \pm 1$ turn out to be the locations having the maximum value of the probability density $\psi_{1}^{*} \psi_{1}$, as stated in Theorem 2.2 and illustrated in Fig. $1 \mathrm{~b}$.

Eigen-trajectories in $n=1$ state can be categorized into three sets $\Omega_{1}, \Omega_{2}$, and $\Omega_{3}$ : $\Omega_{1}$ contains all the trajectories encircling $\bar{x}_{\text {eq }}=-1, \Omega_{2}$ contains all the trajectories encircling $\bar{x}_{\mathrm{eq}}=1$, and $\Omega_{3}$ contains all the trajectories encircling both $\bar{x}_{\mathrm{eq}}=-1$ and $\bar{x}_{\text {eq }}=1$, as shown in Fig. 3a. It is interesting to find that the periods of quantum harmonic oscillator are quantized according to these three sets of trajectories. With the help of Cauchy theorem, the period $\bar{T}$ of the $n=1$ harmonic oscillator can be evaluated from the contour integration along any closed complex contour $c$ traced by the particle

$$
\bar{T}=\oint_{c} \mathrm{~d} \bar{t}=\frac{1}{\mathrm{i}} \oint_{c} \frac{\bar{x}}{\bar{x}^{2}-1} \mathrm{~d} \bar{x}=\left\{\begin{array}{cc}
\pi, & \forall c \in \Omega_{1} \text { or } \Omega_{2} \\
2 \pi, & \forall c \in \Omega_{3}
\end{array} .\right.
$$


a Eigentrajectories

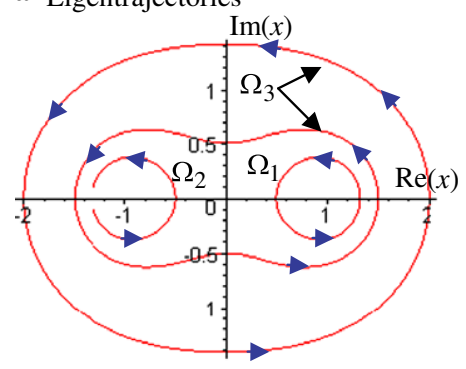

c The mapping of $\Omega_{2}$ in $\psi_{1}$ plane

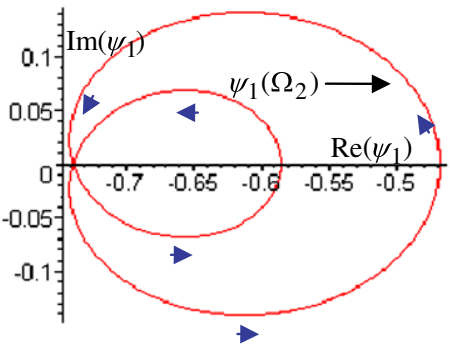

b The mapping of $\Omega_{1}$ in $\psi_{1}$ plane

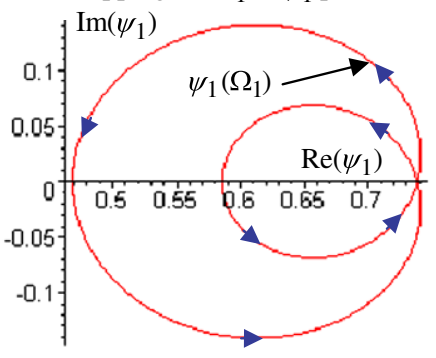

d The mapping of $\Omega_{3}$ in $\psi_{1}$ plane

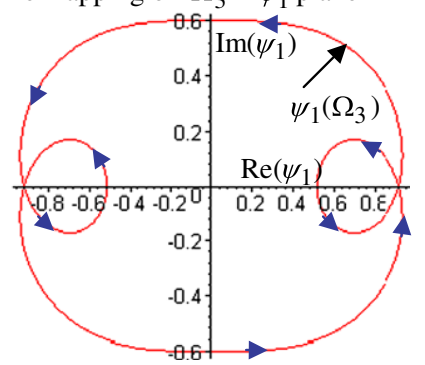

Fig. 3. (a) Eigen-trajectories. Eigen-trajectories in the complex $x$ plane for $n=1$ eigenstate of harmonic oscillator can be categorized into three sets: $\Omega_{1}$ contains all the trajectories encircling $\bar{x}_{\text {eq }}=-1, \Omega_{2}$ contains all the trajectories encircling $\bar{x}_{\mathrm{eq}}=1$, and $\Omega_{3}$ contains all the trajectories encircling both $\bar{x}_{\mathrm{eq}}=-1$ and $\bar{x}_{\mathrm{eq}}=1$. (b) The mapping of $\Omega_{1}$ in $\psi_{1}$ plane. The eigenfunction $\psi_{1}(x)$ serves as a conformal mapping from the complex $x$ plane to the complex $\psi_{1}$ plane and transforms the trajectory set $\Omega_{1}$ in the $x$ plane to the trajectory set $\psi_{1}\left(\Omega_{1}\right)$ in the $\psi_{1}$ plane. Since $\psi_{1}\left(\Omega_{1}\right)$ does not enclose the origin of the $\psi_{1}$ plane, the action variable $J$ evaluated along $\Omega_{1}$ is zero according to Theorem 3.2. (c) The mapping of $\Omega_{2}$ in $\psi_{1}$ plane. There is no encirclement of the origin by $\psi_{1}\left(\Omega_{2}\right)$, indicating the action variable $J$ evaluated along $\Omega_{2}$ is also zero. (d) The mapping of $\Omega_{3}$ in $\psi_{1}$ plane.The action variable $J$ evaluated along $\Omega_{3}$ is $h$ by noting that there is one encirclement of the origin by the contour $\psi_{1}\left(\Omega_{3}\right)$.

All trajectories encircling only one of the equilibrium point thus have period $T=\bar{T} / \omega=\pi \sqrt{m / k}$ and those encircling both of the equilibrium point have period $T=2 \pi \sqrt{m / k}$. In short, although there are infinitely many possible eigen-trajectories in the $n=1$ eigenstate, only two discrete values are allowed for the period of oscillation in this state.

We proceed to consider the validity of the quantization rule proposed by Sommerfeld and Wilson in the $n=1$ eigenstate. Evaluating the contour integration of $\bar{p}$ along any eigen-trajectory $c$ by residue theorem, we obtain

$$
\oint_{c} p \mathrm{~d} x=\hbar \oint_{c} \bar{p} \mathrm{~d} \bar{x}=\mathrm{i} \hbar \oint_{c} \frac{\bar{x}^{2}-1}{\bar{x}} \mathrm{~d} \bar{x}=\left\{\begin{array}{lc}
0, & \forall c \in \Omega_{1} \text { or } \Omega_{2} \\
h, & \forall c \in \Omega_{3}
\end{array} .\right.
$$

The above result implies that along all the possible closed eigen-trajectories in the $n=1$ eigenstate, the action variable $\phi p \mathrm{~d} x$ has only two discrete values: 0 or $h$. This quantization phenomenon can also be verified graphically by the mapping theory stated in Theorem 3.2. The eigenfunction $\psi_{1}(x)$ serves as a conformal mapping from the complex $x$ plane to the complex $\psi_{1}$ plane and transforms the trajectory sets $\Omega_{1}, \Omega_{2}$, and $\Omega_{3}$ in the $x$ plane to the trajectory sets $\psi_{1}\left(\Omega_{1}\right), \psi_{1}\left(\Omega_{2}\right)$, and $\psi_{1}\left(\Omega_{3}\right)$ in the $\psi_{1}$ plane. Since $\psi_{1}\left(\Omega_{1}\right)$ and $\psi_{1}\left(\Omega_{2}\right)$ do not 
enclose the origin of the $\psi_{1}$ plane (refer to Figs. $3 \mathrm{~b}$ and c), the action variable $J$ evaluated along $\Omega_{1}$ or $\Omega_{2}$ is zero according to Theorem 3.2. The action variable $J$ evaluated along $\Omega_{3}$ is $h$ by noting that there is one encirclement of the origin by the contour $\psi_{1}\left(\Omega_{3}\right)$, as shown in Fig. 3d.

Energy distribution within $n=1$ eigenstate is somewhat different from that of ground state. Direct calculation of the terms in Eq. (3.11) using $\bar{p}$ from Eq. (4.6) gives

$$
\frac{1}{2} \bar{p}^{2}+\frac{1}{2} \bar{x}^{2}=1-\frac{1}{2 \bar{x}^{2}}, \quad \bar{Q}=-\frac{\mathrm{i}}{2} \frac{\mathrm{d} \bar{p}}{\mathrm{~d} \bar{x}}=\frac{1}{2}\left(1+\frac{1}{\bar{x}^{2}}\right), \quad \bar{x} \in \mathbf{C} .
$$

As expected, the total energy is a constant $\bar{E}=\bar{p}^{2} / 2+\bar{x}^{2} / 2+\bar{Q}=3 / 2$ independent of $\bar{x}$, which is equal to the eigenvalue $E=\bar{E} \hbar \omega=(3 / 2) \hbar \omega=E_{1}$ corresponding to the eigenfunction $\psi_{1}$. This pointwise conservation of energy ensures that at every point on each $n=1$ eigen-trajectory, the total energy of the particle is always equal to $(3 / 2) \hbar \omega$. Unlike the case in the ground state, where classical energy $\bar{p}^{2} / 2+\bar{x}^{2} / 2$ is zero and the quantum potential $\bar{Q}$ provides the only source of energy, one can see from Eq. (4.10) that in the first excited state the classical energy and the quantum potential energy are of comparable magnitude.

An intuitive understanding of the particle's motion in complex plane is gained from the consideration of the complex Newton's second law. Inserting $\psi_{1}$ in Eq. (2.7b), one gets

$$
\ddot{\bar{x}}=-\frac{\mathrm{d}}{\mathrm{d} \bar{x}}(\bar{V}+\bar{Q})=-\bar{x}+\frac{1}{\bar{x}^{3}} .
$$

It can be checked that the first integration of the above equation results in Eq. (4.6). Along the eigen-trajectory, we are interested in the points where the classical force $-\partial V / \partial \bar{x}$ is balanced by the quantum force $-\mathrm{d} Q / \mathrm{d} \bar{x}$. By setting the acceleration $\ddot{\bar{x}}$ equal to zero, we have $\bar{x}= \pm 1$, which are just the equilibrium points obtained from Eq. (4.6). As $|\bar{x}| \rightarrow \infty$, the quantum force $-\mathrm{d} Q / \mathrm{d} \bar{x}=1 / \bar{x}^{3}$ approaches to zero and the classical equation of motion $\ddot{\bar{x}}=-\bar{x}$ is recovered; while when $\bar{x} \rightarrow 0$, the classical force becomes negligible and the motion is dominated by the quantum force. Fig. 4 demonstrates how the oscillator is moving under the influence of the total potential $V_{\text {Total }}=V+Q$. The action of the quantum potential $Q$ concentrates on the neighborhood surrounding the singularity at the origin, where the quantum force is most significant. For those trajectories far away from the singularity, the oscillator is nearly free from quantum effect and is only subjected to the smoothly changing potential $V=k x^{2} / 2$.

We are now in a position to show that the properties derived from the above two lowest states are general rules for every eigenstate in harmonic oscillators.

Theorem 4.1. The action variable $\oint_{c} p \mathrm{~d} x$ evaluated along any closed eigen-trajectory $c$ of harmonic oscillator satisfies the Sommerfeld-Wilson quantization rule, i.e.,

$$
\oint_{c} p \mathrm{~d} x=n_{c} h, \quad n_{c}=0,1,2, \cdots .
$$

Proof. From Eqs. (2.6) and (4.1), we have

$$
\oint_{c} p \mathrm{~d} x=\frac{h}{2 \pi \mathrm{i}} \oint_{c} \frac{H_{n}^{\prime}(\bar{x})-\bar{x} H_{n}(\bar{x})}{H_{n}(\bar{x})} \mathrm{d} \bar{x}=\frac{h}{2 \pi \mathrm{i}} \oint_{c} \frac{H_{n}^{\prime}(\bar{x})}{H_{n}(\bar{x})} \mathrm{d} \bar{x},
$$

where prime denotes the differentiation with respect to $\bar{x}$ and $c$ is any closed complex contour traced by a particle moving in the $n$th eigenstate. To evaluate the contour integral in 


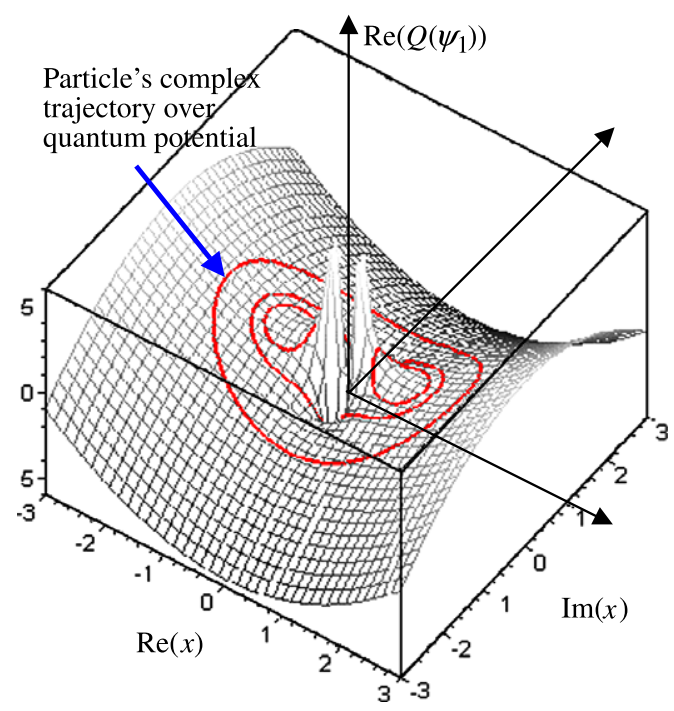

Fig. 4. Harmonic oscillator in the quantum state $n=1$ is moving under the influence of the total potential $V_{\text {Total }}=V+Q$. The action of the quantum potential $Q$ concentrates on the neighborhood surrounding the singularity at the origin, where the quantum force is most significant. For trajectories far away from the singularity, the oscillator is nearly free from quantum effect and is subjected only to the smoothly changing potential $V=k x^{2} / 2$.

Eq. (4.13), we quote a useful theorem from the calculus of residues [6]. Let $\Gamma$ be a simple closed contour with its interior in a domain $D$. Suppose that function $F(z)$ is analytic in $D$, except at some points interior to $\Gamma$ where it has poles, and suppose $F(z) \neq 0, \forall z$ on $\Gamma$. Then

$$
\frac{1}{2 \pi \mathrm{i}} \oint_{\Gamma} \frac{F^{\prime}(z)}{F(z)} \mathrm{d} z=N-P
$$

where $N$ and $P$ are the number of zeros and poles, respectively, of $F(z)$ interior to $\Gamma$, and the integral is taken in the positive direction along $\Gamma$. The multiplicity of each zero and pole is counted in determining $N$ and $P$. Now we apply the above residue theorem to Eq. (4.13). Let $c$ be the closed complex contour traced by a particle in the $n$th eigenstate and define the integer $n_{c}$ as

$$
n_{c}=\text { number of zeros of } H_{n}(\bar{x}) \text { enclosed by } c,
$$

with multiplicities accounted for. Then replacing $F(z)$ with $H_{n}(\bar{x})$ in Eq. (4.14) yields

$$
\frac{1}{2 \pi \mathrm{i}} \oint_{c} \frac{H_{n}^{\prime}(\bar{x})}{H_{n}(\bar{x})} \mathrm{d} \bar{x}=n_{c}, \quad n_{c}=0,1,2, \cdots,
$$

by noting that $H_{n}(\bar{x})$ is an $n$ th-order polynomial without any pole. In conjunction with Eq. (4.16), the action variable in Eq. (4.13) then must assume the quantized values assigned by $n_{c}$.

We thus have proved the Sommerfeld-Wilson quantization rule of action variable under the framework of quantum Hamilton mechanics. The quantization rule (4.12) is not acquirable from standard quantum mechanics, since no sense of trajectory can be defined there; it is also not accessible from Bohmian mechanics, since according to Bohm's prediction, particle in any eigenstate of harmonic oscillator is motionless and no trajectory can be produced. 
The following theorem characterizes the continuous transition from the quantum harmonic oscillator to the classical harmonic oscillator. A more detailed analysis of harmonic oscillator using quantum Hamilton mechanics was treated in [7].

Theorem 4.2. The quantum harmonic oscillator governed by the Hamilton equations (2.7) transits continuously to the classical harmonic oscillator as $n \rightarrow \infty$ and $|\alpha x| \rightarrow \infty$.

Proof. With the understanding from Eq. (2.8) that the quantum Newton equation produces exactly the solution of classical harmonic oscillator when $Q=0$ and $x \in \mathbf{R}$, we shall show that the condition of $\alpha x \rightarrow \infty$ ensures $Q=0$, while the condition of $n \rightarrow \infty$ ensures $x \in \mathbf{R}$. A necessary condition for the motion to fall on real space and continue to stay there is the disappearance of the quantum potential $Q$, since the existence of $Q$ generates a complex force to drive the particle into complex space. From the definition of quantum potential in Eq. (2.11), we have

$$
Q=-\frac{1}{2} \hbar \omega \frac{\mathrm{d}^{2}}{\mathrm{~d} \bar{x}^{2}} \ln \psi(\bar{x})=\frac{1}{2} \hbar \omega\left[1+\left(\frac{H_{n}^{\prime}(\bar{x})}{H_{n}(\bar{x})}\right)^{2}-\frac{H_{n}^{\prime \prime}(\bar{x})}{H_{n}(\bar{x})}\right] .
$$

It can be seen that $Q$ is not zero in any eigenstate. Because both $H_{n}^{\prime}(\bar{x}) / H_{n}(\bar{x})$ and $H_{n}^{\prime \prime}(\bar{x}) / H_{n}(\bar{x})$ are strictly proper rational functions of $\bar{x}$, the quantum potential approaches a constant $Q=\hbar \omega / 2$ as $|\bar{x}|=|\alpha x| \rightarrow \infty$, and then the quantum force $-\mathrm{d} Q / \mathrm{d} x$ disappears such that the equation of quantum motion (2.8) becomes the classical equation of motion $m \ddot{x}+k x=0$; however, in this situation $x$ is still a complex variable rather than a real variable. The condition $|\bar{x}| \rightarrow \infty$ alone is not sufficient to guarantee the transition to the classical equation of motion embedded in real space. The other factor influencing the transition is the quantum number $n$. For large quantum number $n$, Hermite polynomial has the following asymptotic expansion

$$
H_{2 n}(\bar{x})=2(-1)^{n} \frac{(2 n-1) !}{(n-1) !} \mathrm{e}^{\bar{x}^{2} / 2}\left[\cos \sqrt{4 n+1}+\mathrm{O}\left(\frac{1}{\sqrt[4]{n}}\right)\right] .
$$

With this approximation for large $n$, the equation of motion (4.1) becomes

$$
\frac{\mathrm{d} \bar{x}}{\mathrm{~d} \bar{t}}=\mathrm{i} \sqrt{4 n+1} \tan (\sqrt{4 n+1} \bar{x}), \quad \bar{x}=\bar{x}_{\mathrm{R}}+i \bar{x}_{\mathrm{I}} \in \mathbf{C},
$$

which has the solution

$$
\sin \left(\sqrt{4 n+1}\left(\bar{x}_{\mathrm{R}}+\mathrm{i} \bar{x}_{\mathrm{I}}\right)\right)=\left|c_{r}\right| \mathrm{e}^{\mathrm{i}\left((4 n+1) \bar{t}+c_{\theta}\right)}, \quad \bar{x}_{\mathrm{R}}, \bar{x}_{\mathrm{I}} \in \mathbf{R},
$$

where $c_{r}$ and $c_{\theta}$ are integration constants determined from the initial position $\bar{x}_{0}$. Eliminating $\bar{x}_{\mathrm{R}}$ from the real part and the imaginary part of the above equation, we obtain

$$
\frac{\cos ^{2}\left[(4 n+1) \bar{t}+c_{\theta}\right]}{\cosh ^{2}\left(\sqrt{4 n+1} \bar{x}_{\mathrm{I}}\right)}+\frac{\sin ^{2}\left[(4 n+1) \bar{t}+c_{\theta}\right]}{\sinh ^{2}\left(\sqrt{4 n+1} \bar{x}_{\mathrm{I}}\right)}=\frac{1}{\left|c_{r}\right|^{2}} .
$$

Imposing the magnitude constraint of the cosine function yields the allowable $\bar{x}_{\mathrm{I}}$ in the range of

$$
\left|\bar{x}_{\mathrm{I}}(\bar{t})\right| \leqslant \frac{1}{\sqrt{4 n+1}} \cosh ^{-1}\left(\sqrt{1+\left|c_{r}\right|^{2}}\right), \quad \forall t \geqslant 0 .
$$




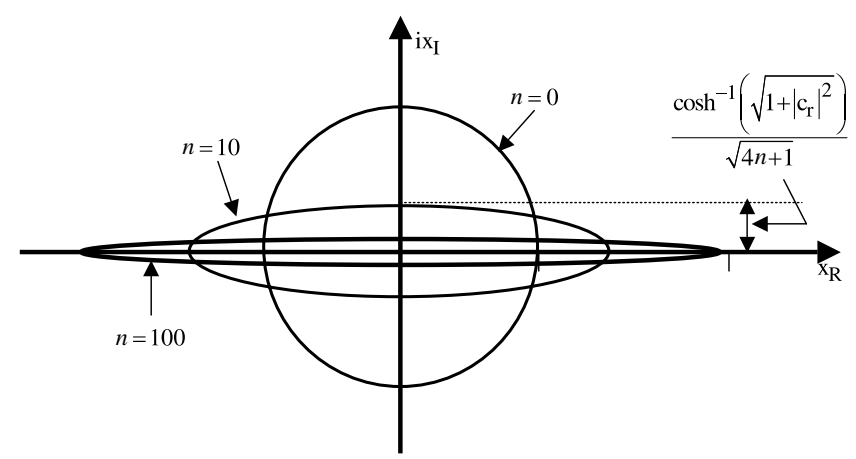

Fig. 5. Complex trajectory for three eigenstates, showing that the imaginary motion $x_{I}$ is suppressed gradually with increasing quantum number $n$ and eventually, the trajectory completely falls within real space as $n \rightarrow \infty$.

Therefore, for a given initial condition we have

$$
\lim _{n \rightarrow \infty}\left|\bar{x}_{\mathrm{I}}(\bar{t})\right|=0, \quad \forall t \geqslant 0,
$$

which implies that as $n \rightarrow \infty$, the imaginary part of the motion vanishes and the trajectory eventually falls on the real axis. Fig. 5 demonstrates the decreasing magnitude of the imaginary motion with increasing quantum number $n$. To summarize, the condition $|\alpha x| \rightarrow \infty$ ensures the nullity of the quantum force, while the condition $n \rightarrow \infty$ nullifies the imaginary part of the complex motion. The combination of these two conditions therefore results in the exact classical motion.

\section{Tunneling in quantum Hamilton mechanics}

Let $\psi$ be the wavefunction describing a particle's tunneling motion in a classically prohibited region. Quantum mechanics adopts $\psi^{*} \psi$ to predict the possible locations of the particle in the tunneling region, whereas in quantum Hamilton mechanics, we regard $\psi$ as a dynamic system whose behavior obeys the Hamilton equations of motion. Using dynamic representation of $\psi$, the tunneling dynamics can be modeled exactly by quantum Hamilton equations without any approximation. The advantage of solving tunneling problems by Hamilton mechanics is twofold. It makes the tunneling time as simple as the usual classical time without the necessity of defining any time operator [8] and second, it provides the tunneling trajectory in an unambiguous way such that the trajectories in classical regions and nonclassical regions can be connected smoothly.

Here, we are concerned with the tunneling motion of a particle under the action of the potential step given by

$$
V(x)= \begin{cases}V_{0} & x_{\mathrm{R}}>0, \\ 0 & x_{\mathrm{R}}<0,\end{cases}
$$

where the potential height $V_{0}$ is greater than the particle's total energy $E$ (see Fig. 6a). Particle with energy $E$ moving within a step potential with height $V_{0}>E$ is classically prohibited, since this would lead to an imaginary momentum $p=\sqrt{2 m\left(E-V_{0}\right)}=\mathrm{i} \sqrt{2 m\left(V_{0}-E\right)}$ within the potential; however, this prohibition is no longer necessary in quantum Hamilton mechanics where particle's motion is already defined in complex domain. Actually, the 
$\mathbf{a}$

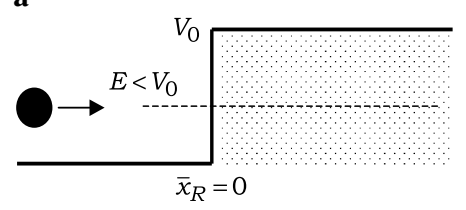

b

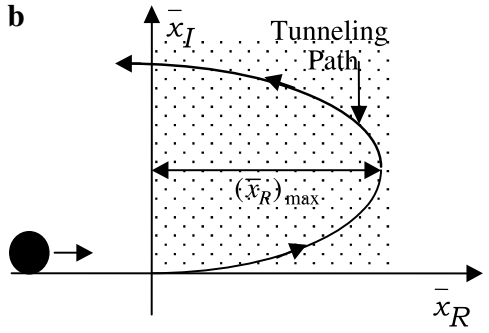

c

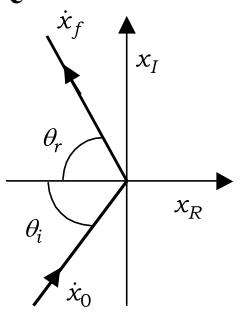

Fig. 6. (a) A particle with total energy $E$ incident on a step potential with height $V_{0}>E$. (b) The tunneling path within the classically prohibited region shows the reversion of the moving direction at the turning point. (c) The incident velocity $x_{0}{ }_{0}$ and the reflected velocity $x_{{ }_{\mathrm{f}}}$ obey the reflection law $\theta_{\mathrm{i}}=\theta_{\mathrm{f}}$.

wave-particle duality is a natural outcome of particle motion in complex space [9]. Our aim here is to find the actual trajectory $x(t)$ as a function of the classical time $t$ in the classically prohibited region $x_{\mathrm{R}}>0$ and to examine how this nonclassical trajectory is connected to the classical region $x_{\mathrm{R}}<0$. The Schrödinger equation for step potential has the solution:

$$
\begin{array}{ll}
\psi(x)=A \mathrm{e}^{\mathrm{i} k_{1} x}+B \mathrm{e}^{-\mathrm{i} k_{1} x}, & \operatorname{Re}(x)<0, \\
\psi(x)=C \mathrm{e}^{-k_{2} x}+D \mathrm{e}^{k_{2} x}, & \operatorname{Re}(x)>0,
\end{array}
$$

where $k_{1}=\sqrt{2 m E} / \hbar$ and $k_{2}=\sqrt{2 m\left(V_{0}-E\right)} / \hbar$. Standard quantum mechanics suggests that we take $D=0$ to keep $\psi$ from diverging for large $x$. The continuity of $\psi$ and $\mathrm{d} \psi /$ $\mathrm{d} x$ at $x=0$ gives

$$
\frac{B}{A}=-\frac{1+\mathrm{i} k_{1} / k_{2}}{1-\mathrm{i} k_{1} / k_{2}}, \quad \frac{C}{A}=1+\frac{B}{A}=\frac{-2 \mathrm{i} k_{1} / k_{2}}{1-\mathrm{i} k_{1} / k_{2}} .
$$

Therefore, the reflection coefficient becomes $R=(B / A)(B / A)^{*}=1$, which implies that an infinitely wide barrier reflects all the incoming particles with energy below the barrier height, in agreement with the classical prediction. Nevertheless, there is a nonzero wave motion in the step region $x_{\mathrm{R}}>0$, since $C / A \neq 0$. It indicates that although a particle can penetrate the potential step to a certain depth, it will reverse the direction of motion at some turning point, and eventually return to the region $x_{\mathrm{R}}<0$, producing no net transmission of particles to the right region of the step. The above prediction from standard quantum mechanics is experimentally consistent but not precise enough to demonstrate clearly what is really happened within the step potential, such as the location of the turning point, the time spent and most importantly, the trajectory within the step potential. All these detailed information of tunneling can be accessed by solving Hamilton equations (1.9).

It has been shown in Theorem 2.1 that the solution for Eq. (1.9b) is given by $p=-\mathrm{i} \hbar \mathrm{d}(\ln \psi) / \mathrm{d} x$, where for tunneling problem $\psi$ is given by Eq. (5.2). Using this $p$ in Eq. (1.9a), we obtain

$$
\begin{aligned}
& \frac{\mathrm{d} \bar{x}}{\mathrm{~d} \bar{t}}=\frac{\alpha_{1} \mathrm{e}^{\mathrm{i} \bar{x}}-\mathrm{e}^{-\mathrm{i} \bar{x}}}{\alpha_{1} \mathrm{e}^{\mathrm{i} \bar{x}}+\mathrm{e}^{-\mathrm{i} \bar{x}}}, \quad \operatorname{Re}(\bar{x})<0, \\
& \frac{\mathrm{d} \bar{x}}{\mathrm{~d} \bar{t}}=\frac{n}{\mathrm{i}} \frac{\alpha_{2} \mathrm{e}^{n \bar{x}}-\mathrm{e}^{-n \bar{x}}}{\alpha_{2} \mathrm{e}^{n \bar{x}}+\mathrm{e}^{-n \bar{x}}}, \quad \operatorname{Re}(\bar{x})>0, n=\sqrt{\frac{V_{0}}{E}-1},
\end{aligned}
$$


where the dimensionless variables are defined as $\bar{x}=(\sqrt{2 m E} / \hbar) x$ and $\bar{t}=(2 E / \hbar) t$ with $\alpha_{1}=B / A$ and $\alpha_{2}=D / C$; the parameter $n$ has an effect analogous to refractive index. The integration of Eq. (5.4b) has an analytical expression

$$
\alpha_{2} \mathrm{e}^{n \bar{x}}-\mathrm{e}^{-n \bar{x}}=\beta_{2} \mathrm{e}^{-\mathrm{i} n^{2} \bar{t}}
$$

where the constant $\alpha_{2}$ and $\beta_{2}$ can be determined by the incident conditions $\bar{x}(0)=\bar{x}_{\text {in }}$ and $\dot{\bar{x}}(0)=\dot{\bar{x}}_{\text {in }}$ :

$$
\alpha_{2}=\frac{1+\mathrm{i} \dot{\bar{x}}_{\text {in }} / n}{1-\mathrm{i} \dot{\bar{x}}_{\text {in }} / n} \mathrm{e}^{-2 n \bar{x}_{\text {in }}}, \quad \beta_{2}=\frac{2 \mathrm{i} \dot{\bar{x}}_{\text {in }} / n}{1-\mathrm{i} \dot{\bar{x}}_{\text {in }} / n} \mathrm{e}^{-n \bar{x}_{\text {in }}} .
$$

Eq. (5.5) gives the particle's complex position $\bar{x}=\bar{x}_{\mathrm{R}}+\mathrm{i} \bar{x}_{\mathrm{I}}$ as a function of the classical time $\bar{t}$,

$$
\bar{x}(\bar{t})=\bar{x}_{\mathrm{R}}(\bar{t})+\mathrm{i} \bar{x}_{\mathrm{I}}(\bar{t})=\frac{1}{n} \ln \left(\frac{\beta_{2}}{2 \alpha_{2}} \mathrm{e}^{-\mathrm{i} n^{2} \bar{t}} \pm \frac{1}{2 \alpha_{2}} \sqrt{\beta_{2}^{2} \mathrm{e}^{-2 \mathrm{i} n^{2} \bar{t}}+4 \alpha_{2}}\right),
$$

which tells us the arrival time at any position $\bar{x}$. The trajectory in the complex plane can be derived by eliminating time $\bar{t}$ from the real and imaginary parts of Eq. (5.5):

$$
\cosh \left(\alpha_{2 r}+2 n \bar{x}_{\mathrm{R}}\right)=\cos \left(\alpha_{2 \theta}+2 n \bar{x}_{\mathrm{I}}\right)+\frac{1}{2}\left|\beta_{2}\right|^{2} \mathrm{e}^{-\alpha_{22}},
$$

where we have rewritten $\alpha_{2}$ as $\alpha_{2}=\mathrm{e}^{\alpha_{2 r}+\mathrm{i} \alpha_{2 \theta}}, \alpha_{2 r}, \alpha_{2 \theta} \in \mathbf{R}$. A typical tunneling path within the classically prohibited region is depicted in Fig. 6b, showing the reversion of the moving direction at the turning point where $\bar{x}_{\mathrm{R}}$ achieves the maximum value. From Eq. (5.8) we can derive a succinct formula for the penetration depth.

Theorem 5.1. The penetration depth for a particle with mass $m$ and energy $E$ in a step potential with height $V_{0}>E$ is given by

$$
\delta_{\mathrm{p}}=\frac{\hbar}{2 n \sqrt{2 m E}}\left[-\ln \left|\frac{n+\mathrm{i} \dot{\bar{x}}_{\text {in }}}{n-\mathrm{i} \dot{\bar{x}}_{\text {in }}}\right|+\cosh ^{-1}\left(1+\frac{2\left|\dot{\bar{x}}_{\text {in }}\right|^{2}}{\left|n^{2}+\dot{\bar{x}}_{\text {in }}^{2}\right|}\right)\right],
$$

where $\dot{\bar{x}}_{\mathrm{in}}$ is the incident velocity and $n=\sqrt{V_{0} / E-1}$ is the refractive index.

Proof. Eq. (5.8) reveals that the real part of the position $\bar{x}_{\mathrm{R}}$ is restricted to the following range

$$
\cosh \left(\alpha_{2 r}+2 n \bar{x}_{\mathrm{R}}\right) \leqslant 1+\left|\beta_{2}\right|^{2} \mathrm{e}^{-\alpha_{2 r}} / 2
$$

from which the maximum allowable $\bar{x}_{\mathrm{R}}$ can be found as

$$
\left(\bar{x}_{\mathrm{R}}\right)_{\max }=\frac{1}{2 n}\left[-\alpha_{2 r}+\cosh ^{-1}\left(1+\frac{1}{2}\left|\beta_{2}\right|^{2} \mathrm{e}^{-\alpha_{2 r}}\right)\right] .
$$

This is just the penetration depth $\delta_{\mathrm{p}}$ in the step potential. Substituting the definitions for $\alpha_{2}$ and $\beta_{2}$ from Eq. (5.6), we arrive at the express $\delta_{\mathrm{p}}$ in the form of Eq. (5.9).

Eq. (5.9) indicates that the penetration depth $\delta_{\mathrm{p}}$ depends on the incident velocity $\dot{\bar{x}}_{\text {in }}$ and the refractive index $n$. The unobservability of $\operatorname{Im}\left(\dot{\bar{x}}_{\text {in }}\right)$ produces the uncertainty in the measurement of $\delta_{\mathrm{p}}$; nevertheless, Eq. (5.9) reveals how $\delta_{\mathrm{p}}$ is influenced by $\operatorname{Im}\left(\dot{\bar{x}}_{\text {in }}\right)$ and tell us the possible range of $\delta_{\mathrm{p}}$ due to the variation of $\operatorname{Im}\left(\dot{\bar{x}}_{\text {in }}\right)$. It can be seen that the rise and fall 
of the surface plot of $\delta_{\mathrm{p}}\left(\operatorname{Re}\left(\dot{\bar{x}}_{\text {in }}\right), \operatorname{Im}\left(\dot{\bar{x}}_{\text {in }}\right)\right)$ is significant only in the region where $\operatorname{Re}\left(\dot{\bar{x}}_{\text {in }}\right)$ is near zero and $-n \leqslant \operatorname{Im}\left(\dot{\bar{x}}_{\text {in }}\right) \leqslant n$. The maximum $\delta_{\mathrm{p}}$ occurs at the incident velocity $\dot{\bar{x}}_{\text {in }}=n \mathrm{i}$ for which the particle can penetrate the nonclassical region by an infinite depth, however, this special incident velocity has only imaginary component and cannot be realized in the real world. For the velocity regions satisfying $\left|\dot{\bar{x}}_{\text {in }}\right| \gg n, \delta_{\mathrm{p}}$ is a constant independent of $\operatorname{Re}\left(\dot{\bar{x}}_{\text {in }}\right)$ and $\operatorname{Im}\left(\dot{\bar{x}}_{\text {in }}\right)$, being equal to

$$
\delta_{\mathrm{p}}=\frac{\hbar \cosh ^{-1}(3)}{2 \sqrt{2 m\left(V_{0}-E\right)}} \approx 0.8814 \frac{\hbar}{\sqrt{2 m\left(V_{0}-E\right)}},
$$

where $\hbar / \sqrt{2 m\left(V_{0}-E\right)}=1 / k_{2}$ is the penetration depth predicted by the standard quantum mechanics.

Let $\dot{\bar{x}}_{\text {in }}$ and $\dot{\bar{x}}_{\text {out }}$ be the incident and the reflected velocities, respectively, as shown in Fig. 6c. Using $\dot{\bar{x}}_{\text {in }}$ and $\dot{\bar{x}}_{\text {out }}$, we define the angle-of-incident $\theta_{\mathrm{i}}$ and the angle-of-reflection $\theta_{\mathrm{r}}$, respectively, as

$$
\tan \theta_{\mathrm{i}}=\frac{\operatorname{Im}\left(\dot{\overline{\bar{x}}}_{\text {in }}\right)}{\operatorname{Re}\left(\dot{\bar{x}}_{\text {in }}\right)}, \quad \tan \theta_{\mathrm{r}}=-\frac{\operatorname{Im}\left(\dot{\bar{x}}_{\text {out }}\right)}{\operatorname{Re}\left(\dot{\bar{x}}_{\text {out }}\right)} .
$$

Then it can be shown that the tunneling particle exhibits wave-like behavior obeying the reflection law.

Theorem 5.2. A particle with energy $E$ incident upon a step potential $V_{0}>E$ obeys the reflection law,

$$
\theta_{\mathrm{i}}=\theta_{\mathrm{r}} \text {. }
$$

Proof. Let $\bar{t}_{\text {out }}$ be the instant that the particle goes out of the step potential and returns to the starting point $\bar{x}_{\mathrm{R}}=0$, i.e., $\bar{x}_{\mathrm{R}}\left(\bar{t}_{\text {out }}\right)=0$. Evaluating Eq. (5.8) at $\bar{t}=\bar{t}_{\text {out }}$ yields

$$
\cosh \left(\alpha_{2 r}\right)=\cos \left(\alpha_{2 \theta}+2 n \bar{x}_{\mathrm{I}}\left(\bar{t}_{\text {out }}\right)\right)+\frac{1}{2}\left|\beta_{2}\right|^{2} \mathrm{e}^{-\alpha_{2 r}},
$$

where $\bar{x}_{\mathrm{I}}\left(\bar{t}_{\text {out }}\right)$ has the solution

$$
\bar{x}_{\mathrm{I}}\left(\bar{t}_{\text {out }}\right)=0 \text { or }-\alpha_{2 \theta} / n \text {. }
$$

The solution $\bar{x}_{\mathrm{I}}\left(\bar{t}_{\text {out }}\right)=0$ corresponds to the initial incident position and $\bar{x}_{\mathrm{I}}\left(\bar{t}_{\text {out }}\right)=-\alpha_{2 \theta} / n$ to the exit of the tunneling path, since at the two positions we both have $\bar{x}_{\mathrm{R}}=0$ (see also Fig. 6b). Evaluating Eq. (5.4b) at $\bar{t}=\bar{t}_{\text {out }}$ and applying the condition $\bar{x}\left(\bar{t}_{\text {out }}\right)=-\mathrm{i} \alpha_{2 \theta} / n$, we have

$$
\dot{\bar{x}}_{\text {out }}=\dot{\bar{x}}\left(\bar{t}_{\text {out }}\right)=\frac{n}{\mathrm{i}} \frac{\alpha_{2} \mathrm{e}^{-\mathrm{i} \alpha_{2 \theta}}-\mathrm{e}^{\mathrm{i} \alpha_{2 \theta}}}{\alpha_{2} \mathrm{e}^{-\mathrm{i} \alpha_{2 \theta}}+\mathrm{e}^{\mathrm{i} \alpha_{2 \theta}}}=\frac{n}{\mathrm{i}} \frac{\alpha_{2}^{*}-1}{\alpha_{2}^{*}+1} .
$$

On the other hand, the evaluation of Eq. (5.4b) at $\bar{t}=\bar{t}_{\text {in }}=0$ gives

$$
\dot{\bar{x}}_{\text {in }}=\dot{\bar{x}}\left(\bar{t}_{\text {in }}\right)=\frac{n}{\mathrm{i}} \frac{\alpha_{2} \mathrm{e}^{-\mathrm{i} \alpha_{2 \theta}}-\mathrm{e}^{\mathrm{i} \alpha_{2 \theta}}}{\alpha_{2} \mathrm{e}^{-\mathrm{i} \alpha_{2 \theta}}+\mathrm{e}^{\mathrm{i} \alpha_{2 \theta}}}=\frac{n}{\mathrm{i}} \frac{\alpha_{2}-1}{\alpha_{2}+1} .
$$

Comparing Eq. (5.16) with Eq. (5.17), we obtain a critical relation

$$
\dot{\bar{x}}_{\text {in }}=-\dot{\bar{x}}_{\text {out }}^{*},
$$

which is the reflection law of tunneling in complex plane. When expressed in component form, it becomes 


$$
\operatorname{Re}\left(\dot{\bar{x}}_{\text {out }}\right)=-\operatorname{Re}\left(\dot{\bar{x}}_{\text {in }}\right), \quad \operatorname{Im}\left(\dot{\bar{x}}_{\text {out }}\right)=\operatorname{Im}\left(\dot{\bar{x}}_{\text {in }}\right) .
$$

which implies that the imaginary components of the incident and the reflected velocities are the same, while the real components differ only in sign. Applying the condition (5.19) to the definition (5.12) yields the reflection law $\theta_{\mathrm{i}}=\theta_{\mathrm{r}}$.

Next, we proceed to find the time spent in the nonclassical region $\bar{x}_{\mathrm{R}}>0$. The tunneling time $\bar{t}_{\mathrm{f}}$ within the step potential can be found by evaluating Eq. (5.5) at $\bar{t}=\bar{t}_{\text {out }}$ and using the results $\bar{x}\left(\bar{t}_{\text {out }}\right)=-\mathrm{i} \alpha_{2 \theta} / n$ :

$$
\mathrm{e}^{-\mathrm{in} h^{2} \bar{t}_{\text {out }}}=\frac{\alpha_{2}^{*}-1}{\alpha_{2}-1} \mathrm{e}^{\mathrm{i} \alpha_{2 \theta}}
$$

Solving for $\bar{t}_{\text {out }}$ yields

$$
\bar{t}_{\text {out }}=\frac{2 l \pi-\left(\gamma+\alpha_{2 \theta}\right)}{n^{2}},
$$

where $l$ is the minimum integer ensuring $\bar{t}_{\text {out }}>0$ and $\gamma$ is the angle between $\alpha_{2}^{*}-1$ and $\alpha_{2}-1$, i.e., $\mathrm{e}^{\mathrm{i} \gamma}=\left(\alpha_{2}^{*}-1\right) /\left(\alpha_{2}-1\right)$.

When inspecting the tunneling trajectory, one is naturally led to the question why the particle should turn back after reaching $\left(\bar{x}_{\mathrm{R}}\right)_{\max }$. It seems that there is a strong retarding force to prohibit further penetration. This retarding force actually exists, which is furnished by the combined effect of the applied step potential $V_{0}$ and the quantum potential $Q$. The total potential $Q+V_{0}$ is given by Eq. (2.15) with $\psi$ coming from Eq. (5.2b)

$$
\bar{V}_{\text {Total }}=\left(Q+V_{0}\right) / E=1+\left(\frac{\mathrm{d}}{\mathrm{d} \bar{x}} \ln \psi(\bar{x})\right)^{2}=1+n^{2}\left(\frac{\alpha_{2} \mathrm{e}^{n \bar{x}}-\mathrm{e}^{-n \bar{x}}}{\alpha_{2} \mathrm{e}^{n \bar{x}}+\mathrm{e}^{-n \bar{x}}}\right)^{2} .
$$

The change of $\bar{V}_{\text {Total }}$ over the complex plane $\bar{x}=\bar{x}_{\mathrm{R}}+\mathrm{i} \bar{x}_{\mathrm{I}}$ is illustrated in Fig. 7, where we can see that $\bar{V}_{\text {Total }}$ becomes steeper for larger $\bar{x}_{\mathrm{R}}$. It is this increasingly steep potential that forces the particle to turn back at some turning point. As can be observed in Fig. 7, when the tunneling path reaches the turning point, the particle climbs to the highest position of its trajectory over the total potential surface; after passing the turning point, the total potential is decreasing and the particle accelerates toward the exit of the tunneling path. The deceleration and acceleration of the tunneling particle are best described by the quantum Newton law:

$$
\frac{\mathrm{d}^{2} \bar{x}}{\mathrm{~d} \bar{t}^{2}}=-\frac{\mathrm{d} \bar{V}_{\text {Total }}}{\mathrm{d} \bar{x}}=-n^{2} \frac{\mathrm{d}}{\mathrm{d} \bar{x}}\left[\left(\frac{\alpha_{2} \mathrm{e}^{n \bar{x}}-\mathrm{e}^{-n \bar{x}}}{\alpha_{2} \mathrm{e}^{n \bar{x}}+\mathrm{e}^{-n \bar{x}}}\right)^{2}\right] .
$$

It is noticed from the above expression that $\bar{V}_{\text {Total }}$ is proportional to $n^{2}$, which implies that for small $n$ the particle encounters less retarding force, and hence deeper penetration is possible. This effect is also reflected in Eq. (5.10), which shows that large penetration depth is achieved for small $n$. If we set $\alpha_{2}=D / C=0$, then Eq. (5.23) indicates that the retarding force within the step potential is completely zero. Hence, the inclusion of term $\mathrm{e}^{n \bar{x}}$ in Eq. (5.2b) with $D \neq 0$ has the action of providing a retarding force in the positive $\bar{x}_{\mathrm{R}}$ direction and makes the particle turn back to the region $\bar{x}_{\mathrm{R}}<0$. 


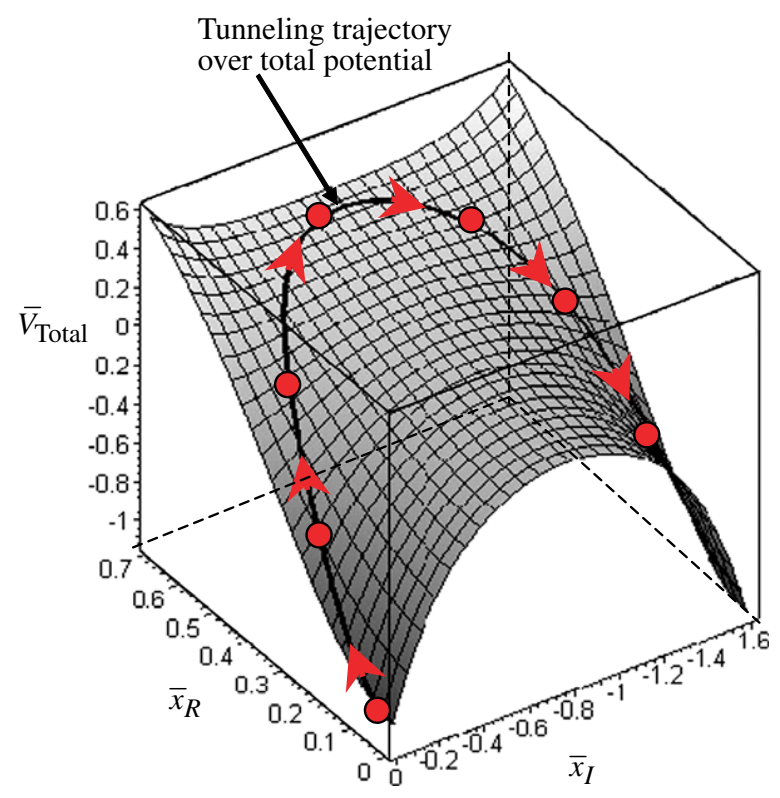

Fig. 7. Total potential confronting the tunneling particle and the resulting tunneling trajectory over it. The increasingly steep total potential forces the particle to turn back at the turning point. After passing the turning point, the total potential is decreasing and the particle accelerates toward the exit of the tunneling path.

The remaining problem to be solved is the connection between classical and nonclassical regions. Motions in the classical region $\bar{x}<0$ and in the nonclassical region $\bar{x}>0$ are governed, respectively, by Eqs. (5.4a) and (5.4b), which, after integration, give

$$
\begin{aligned}
& \alpha_{1} \mathrm{e}^{\mathrm{i} \bar{x}}-\mathrm{e}^{-\mathrm{i} \bar{x}}=\beta_{1} \mathrm{e}^{\mathrm{i} \bar{t}}, \quad \operatorname{Re}(\bar{x})<0, \\
& \alpha_{2} \mathrm{e}^{n \bar{x}}-\mathrm{e}^{-n \bar{x}}=\beta_{2} \mathrm{e}^{-\mathrm{i} n^{2} \bar{t}}, \quad \operatorname{Re}(\bar{x})>0 .
\end{aligned}
$$

Analogous to classical mechanics, the connection between the two regions is established by the continuity of position and velocity evaluated at the both sides of $\bar{x}_{\mathrm{R}}=0$ :

$$
\bar{x}^{-}\left(\bar{t}_{\text {in }}\right)=\bar{x}^{+}\left(\bar{t}_{\text {in }}\right), \quad \dot{\bar{x}}^{-}\left(\bar{t}_{\text {in }}\right)=\dot{\bar{x}}^{+}\left(\bar{t}_{\text {in }}\right),
$$

where $\bar{x}^{-}$and $\bar{x}^{+}$denote the trajectories in the region $\bar{x}_{\mathrm{R}}<0$ and $\bar{x}_{\mathrm{R}}>0$, respectively; $\bar{t}_{\text {in }}$ is the time of particle's incidence upon the step back. In this continuity condition, $\dot{\bar{x}}^{-}\left(\bar{t}_{\text {in }}\right)$ and $\dot{\bar{x}}^{+}\left(\bar{t}_{\text {in }}\right)$ are given by Eq. (5.4), while $\bar{x}^{-}\left(\bar{t}_{\text {in }}\right)$ and $\bar{x}^{+}\left(\bar{t}_{\text {in }}\right)$ are given by Eq. (5.24). The following lemma relates continuity of $\psi$ and $\mathrm{d} \psi / \mathrm{d} x$ at $x_{\mathrm{R}}=0$ to the continuity of $\dot{\bar{x}}$ at $x_{\mathrm{R}}=0$.

Lemma 5.1. For a particle whose motion is described by $\psi$, its velocity is continuous at the interface of two media, if and only if $\psi$ and $\mathrm{d} \psi / \mathrm{d} x$ are continuous at the interface.

Proof. Let $x_{0}$ be the location of interface. Then from Eq. (4.1), we have

$$
\frac{\mathrm{d} x}{\mathrm{~d} t}=-\left.\mathrm{i} \frac{\hbar}{m} \frac{1}{\psi\left(x_{0}\right)} \frac{\mathrm{d} \psi(x)}{\mathrm{d} x}\right|_{x_{0}},
$$

which clearly indicates that the continuity of velocity and the continuity of $\psi$ and $\mathrm{d} \psi / \mathrm{d} x$ are equivalent. 
The continuity of $\psi$ and $\mathrm{d} \psi / \mathrm{d} x$, which is critical in quantum mechanics in deducing the allowable quantization of energy levels, is therefore an alternative expression of continuity of velocity. In the following example, we will determine a global trajectory connecting classical and nonclassical regions by using the continuity condition (5.25).

Example 5.1. Consider a particle starting from the classical region with the initial conditions $\bar{x}(0)=-\pi$ and $\dot{\bar{x}}(0)=1+\mathrm{i} / 2$, going into the classically forbidden region, and then turning back to the classical region again. Accordingly, the trajectory can be divided into three segments:

(1) $0 \leqslant \bar{t} \leqslant \bar{t}_{\text {in }}$ : This part of trajectory, which belongs to a free-particle motion before the entrance to the step potential, is determined by Eq. (5.24a) in the region $\operatorname{Re}(\bar{x})<0$. The two constants $\alpha_{1}$ and $\beta_{1}$ are found from the initial conditions as

$$
\alpha_{1}=\frac{1+\dot{\bar{x}}(0)}{1-\dot{\bar{x}}(0)} \mathrm{e}^{-2 \mathrm{i} \bar{x}(0)}=-1+4 \mathrm{i}, \quad \beta_{1}=\frac{2 \dot{\bar{x}}(0)}{1-\dot{\bar{x}}(0)} \mathrm{e}^{-\mathrm{i} \mathrm{x}(0)}=2-4 \mathrm{i}
$$

and the corresponding trajectory is shown in Fig. 8, wherein the time $\bar{t}_{\text {in }}=\pi$ is the instant at which the particle reaches the boundary $\bar{x}_{\mathrm{R}}=0$, where the particle's position and velocity are given by

$\bar{x}^{-}\left(\bar{t}_{\text {in }}\right)=0, \quad \dot{\bar{x}}^{-}\left(\bar{t}_{\text {in }}\right)=1+\mathrm{i} / 2$.

According to the continuity conditions in Eq. (5.25), the position and velocity evaluated at $\bar{t}_{\text {in }}$ in Eq. (5.28) must be used as the initial conditions for the subsequent tunneling motion in the region $\bar{x}_{\mathrm{R}}>0$.

(2) $\bar{t}_{\text {in }} \leqslant \bar{t} \leqslant \bar{t}_{\text {out }}$ : The tunneling trajectory within the step potential is initiated by the continuity conditions $\bar{x}^{-}\left(\bar{t}_{\text {in }}\right)=\bar{x}^{+}\left(\bar{t}_{\text {in }}\right)=0$ and $\dot{\bar{x}}^{-}\left(\bar{t}_{\text {in }}\right)=\dot{\bar{x}}^{+}\left(\bar{t}_{\text {in }}\right)=1+\mathrm{i} / 2$. By introducing the numerical values of $\bar{x}_{\text {in }}=0$ and $\dot{\bar{x}}_{\text {in }}=1+\mathrm{i} / 2$ in Eq. (5.6), $\alpha_{2}$ and $\beta_{2}$ are found to be (using $n=1$, i.e., $V_{0}=2 E$ in the calculation)

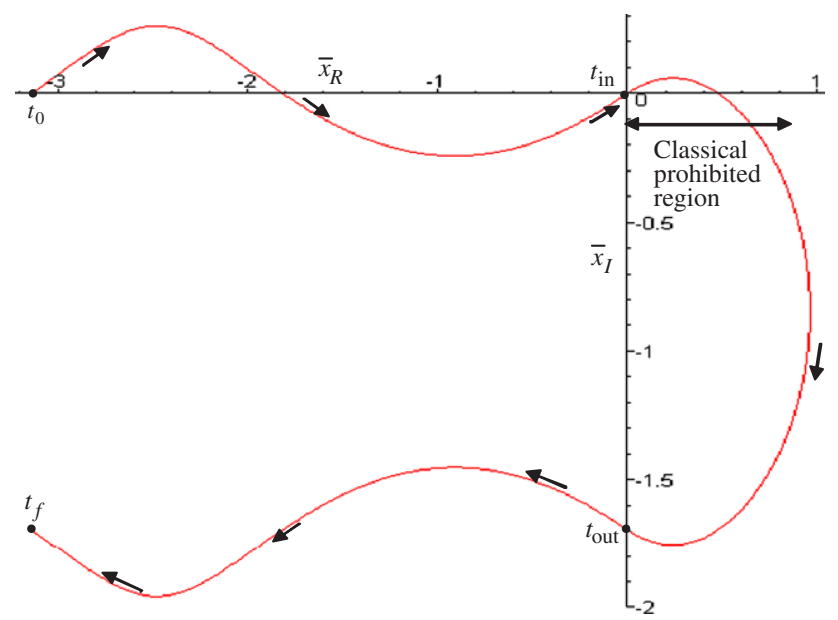

Fig. 8. The global trajectory connecting the classical region $\bar{x}_{\mathrm{R}}<0$ and the nonclassical region $\bar{x}_{\mathrm{R}} \geqslant 0$ by imposing the continuity conditions in position and velocity. In this way, particle's motion before, during, and after the tunneling process can be connected continuously. 
$\alpha_{2}=-\frac{1}{13}+\frac{8}{13} \mathrm{i}, \quad \beta_{2}=-\frac{14}{13}+\frac{8}{13} \mathrm{i}$.

Using $\alpha_{2}$ and $\beta_{2}$ in Eq. (5.24b), the tunneling trajectory in the range of $\operatorname{Re}(\bar{x})>0$ is then determined uniquely as shown in Fig. 8. Regarding this tunneling trajectory, we can find the penetration depth according to Eq. (5.9)

$\delta_{\mathrm{p}}=-\frac{1}{2} \ln \frac{\sqrt{65}}{13}+\frac{1}{2} \cosh ^{-1}\left(1+\frac{2 \sqrt{65}}{13}\right)$.

The end time of the tunneling process $\bar{t}_{\text {out }}$ is evaluated from Eq. (5.21) as

$\bar{t}_{\text {out }}=2 \pi+\tan ^{-1} \frac{16}{37}$.

In addition, the exit position and exit velocity of the tunneling path are given by

$\bar{x}^{+}\left(\bar{t}_{\text {out }}\right)=0+\mathrm{i}\left(-\pi+\tan ^{-1} 8\right), \quad \dot{\bar{x}}^{+}\left(\bar{t}_{\text {out }}\right)=-1+\mathrm{i} / 2$,

which will be treated as the initial conditions of the subsequent trajectory in the range of $\operatorname{Re}(\bar{x})<0$. The reflected velocity from the step potential is given by $\dot{\bar{x}}^{-}\left(\bar{t}_{\text {out }}\right)=\dot{\bar{x}}^{+}\left(\bar{t}_{\text {out }}\right)=-1+\mathrm{i} / 2$, and if we compare $\dot{\bar{x}}^{-}\left(\bar{t}_{\text {out }}\right)$ with the incident velocity $\dot{\bar{x}}^{-}\left(\bar{t}_{\text {in }}\right)=1+\mathrm{i} / 2$ from Eq. (5.28), we obtain $\dot{\bar{x}}^{-}\left(\bar{t}_{\text {in }}\right)=-\dot{\bar{x}}^{-}\left(\bar{t}_{\text {out }}\right)^{*}$ as a confirmation of the reflection law indicated in Eq. (5.18).

(3) $\bar{t}_{\text {out }} \leqslant \bar{t} \leqslant \bar{t}_{\mathrm{f}}$ : This part of trajectory is again a free-particle motion in the range of $\operatorname{Re}(\bar{x})<0$ reflected from the step potential. The initial conditions $\bar{x}^{-}\left(\bar{t}_{\text {out }}\right)$ and $\dot{\bar{x}}^{-}\left(\bar{t}_{\text {out }}\right)$ for this free-particle motion come from the continuity conditions: $\bar{x}^{-}\left(\bar{t}_{\text {out }}\right)=\bar{x}^{+}\left(\bar{t}_{\text {out }}\right)$ and $\dot{\bar{x}}^{-}\left(\bar{t}_{\text {out }}\right)=\dot{\bar{x}}^{+}\left(\bar{t}_{\text {out }}\right)$, from which the new values of $\alpha_{1}$ and $\beta_{1}$ are given by

$\alpha_{1}=\left(-\frac{1}{17}+\frac{4}{17} \mathrm{i}\right) \mathrm{e}^{2 \tan ^{-1} 8-2 \pi}, \quad \beta_{1}=\left(-\frac{18}{17}+\frac{4}{17} \mathrm{i}\right) \mathrm{e}^{\tan ^{-1} 8-\pi}$.

Solving for $\bar{x}(\bar{t})$ from Eq. (5.24a) by using the above values of $\alpha_{1}$ and $\beta_{1}$, we obtain the final portion of the global tunneling trajectory as depicted in Fig. 8. The final time $\bar{t}_{\mathrm{f}}=\pi+\bar{t}_{\text {out }}$ is the time that the particle returns to the starting value of $\bar{x}_{\mathrm{R}}=-\pi$. We thus have connected the nonclassical region with the classical region by imposing the continuity conditions in position and velocity. In this way, particle's motion before, during, and after the tunneling process has been connected continuously.

\section{Observables and operators in spherical coordinates}

The above discussions on quantum Hamilton mechanics and the related quantum problems are all limited to Cartesian coordinates. In this section, we shall continue to demonstrate how quantum operators can be derived directly in spherical coordinates under the framework of Hamilton mechanics. According to the definition of the Quantum Hamiltonian $H$ in Eq. (1.6), we first have to express $\mathbf{p} \cdot \mathbf{p}$ and $\nabla \cdot \mathbf{p}$ in spherical coordinates $(r, \theta, \phi)$ as

$$
\mathbf{p} \cdot \mathbf{p}=p_{r}^{2}+\frac{p_{\theta}^{2}}{r^{2}}+\frac{p_{\phi}^{2}}{r^{2} \sin ^{2} \theta},
$$




$$
\begin{aligned}
\nabla \cdot \mathbf{p} & =\frac{1}{r^{2} \sin \theta}\left[\frac{\partial}{\partial r}\left(r^{2} p_{r} \sin \theta\right)+\frac{\partial}{\partial \theta}\left(p_{\theta} \sin \theta\right)+\frac{\partial}{\partial \phi}\left(\frac{p_{\phi}}{\sin \theta}\right)\right] \\
& =\frac{1}{r^{2} \sin \theta}\left[2 r p_{r} \sin \theta+p_{\theta} \cos \theta+r^{2} \sin \theta \frac{\partial p_{r}}{\partial r}+\sin \theta \frac{\partial p_{\theta}}{\partial \theta}+\frac{1}{\sin \theta} \frac{\partial p_{\phi}}{\partial \phi}\right] .
\end{aligned}
$$

Before evaluating $\partial p_{r} / \partial r, \partial p_{\theta} / \partial \theta$, and $\partial p_{\phi} / \partial \phi$, we need to express $p_{r}, p_{\theta}$, and $p_{\phi}$ as explicit functions of $r, \theta$, and $\phi$. These explicit expressions are afforded by Eq. (1.8) as

$$
p_{r}=\frac{\partial S}{\partial r}=-\mathrm{i} \hbar \frac{\partial \ln \psi}{\partial r}, \quad p_{\theta}=\frac{\partial S}{\partial \theta}=-\mathrm{i} \hbar \frac{\partial \ln \psi}{\partial \theta}, \quad p_{\phi}=\frac{\partial S}{\partial \phi}=-\mathrm{i} \hbar \frac{\partial \ln \psi}{\partial \phi},
$$

where $\psi(r, \theta, \phi)$ is the given wavefunction. By applying Eq. (6.2) to the differentiations involved in Eq. (6.1b), the quantum Hamiltonian $H$ defined in Eq. (1.6) becomes

$$
\begin{aligned}
H= & \frac{1}{2 m}\left[p_{r}^{2}+\frac{\hbar}{\mathrm{i}}\left(\frac{2}{r} p_{r}+\frac{\hbar}{\mathrm{i}} \frac{\partial^{2} \ln \psi}{\partial r^{2}}\right)\right]+\frac{1}{2 m r^{2}}\left[p_{\theta}^{2}+\frac{\hbar}{\mathrm{i}}\left(p_{\theta} \cot \theta+\frac{\hbar}{\mathrm{i}} \frac{\partial^{2} \ln \psi}{\partial \theta^{2}}\right)\right. \\
& \left.+\frac{1}{\sin ^{2} \theta}\left(p_{\phi}^{2}-\hbar^{2} \frac{\partial^{2} \ln \psi}{\partial \phi^{2}}\right)\right]+V(r, \theta, \phi)=\frac{1}{2 m} P_{r}^{2}+\frac{L^{2}}{2 m r^{2}}+V .
\end{aligned}
$$

It can be seen that the Hamiltonian $H$ is uniquely determined by the given wavefunction $\psi(r, \theta, \phi)$. The following theorem gives the dynamic representation of $\psi$ in spherical coordinates.

Theorem 6.1. A quantum state $\psi(r, \theta, \phi)$, expressed in spherical coordinates, is a dynamic system whose behavior obeys the following Hamilton equations:

$$
\begin{aligned}
& \dot{r}=\frac{\hbar}{\mathrm{i} m} \frac{\partial \ln \psi}{\partial r}+\frac{\hbar}{\mathrm{i}} \frac{1}{m r}, \\
& \dot{\theta}=\frac{\hbar}{\mathrm{i} m r^{2}} \frac{\partial \ln \psi}{\partial \theta}+\frac{\hbar}{\mathrm{i}} \frac{\cot \theta}{2 m r^{2}}, \\
& \dot{\phi}=\frac{\hbar}{\mathrm{i} m r^{2} \sin ^{2} \theta} \frac{\partial \ln \psi}{\partial \phi} .
\end{aligned}
$$

Proof. The dynamic representation of the state $\psi$ in spherical coordinates can be derived from Eq. (1.9a) with $H$ given by Eq. (6.3):

$$
\begin{aligned}
& \dot{r}=\frac{\partial H}{\partial p_{r}}=\frac{p_{r}}{m}+\frac{\hbar}{\mathrm{i}} \frac{1}{m r}, \\
& \dot{\theta}=\frac{\partial H}{\partial p_{\theta}}=\frac{p_{\theta}}{m r^{2}}+\frac{\hbar}{\mathrm{i}} \frac{\cot \theta}{2 m r^{2}}, \\
& \dot{\phi}=\frac{\partial H}{\partial p_{\phi}}=\frac{p_{\phi}}{m r^{2} \sin ^{2} \theta} .
\end{aligned}
$$

On deriving the above Hamilton equations, the canonical variables $\mathbf{q}=(r, \theta, \phi)$ and $\mathbf{p}=\left(p_{r}, p_{\theta}, p_{\phi}\right)$ must be regarded as independent variables in the quantum Hamiltonian $H$, as in classical Hamilton mechanics. The adjoin Hamilton equations $\dot{\mathbf{p}}=-\partial H / \partial \mathbf{q}$ from Eq. (1.9b) are redundant, since, according to Theorem 2.1, the solution for $\mathbf{p}$ is already given by Eq. (6.2), as long as $\psi$ is a solution of the Schrödinger equation. By substituting Eq. (6.2) into Eq. (6.5), we obtain the dynamic representation of $\psi$ as in Eq. (6.4). 
In comparison with their classical counterparts, the mechanical momenta $P_{r}^{2}$ and $L^{2}$ in quantum Hamiltonian (6.3) contain additional quantum correction terms:

$$
\begin{aligned}
P_{r}^{2} & =p_{r}^{2}+\frac{\hbar}{\mathrm{i}}\left(\frac{2}{r} p_{r}+\frac{\hbar}{\mathrm{i}} \frac{\partial^{2} \ln \psi}{\partial r^{2}}\right), \\
L^{2} & =p_{\theta}^{2}+\frac{\hbar}{\mathrm{i}}\left(p_{\theta} \cot \theta+\frac{\hbar}{\mathrm{i}} \frac{\partial^{2} \ln \psi}{\partial \theta^{2}}\right)+\frac{L_{z}^{2}}{\sin ^{2} \theta}, \quad L_{z}^{2}=p_{\phi}^{2}-\hbar^{2} \frac{\partial^{2} \ln \psi}{\partial \phi^{2}},
\end{aligned}
$$

where the terms involving Planck constant stem from quantum correction. The corresponding operators for $P_{r}^{2}, L^{2}$, and $L_{z}^{2}$ can be identified by expressing Eq. (6.6) in the form of Eq. (3.1).

Theorem 6.2. The momentum operators $\hat{P}_{r}, \hat{L}_{2}$, and $\hat{L}_{z}$ corresponding to the observables $P_{r}$, $L^{2}$, and $L_{z}$ defined in Eq. (6.6) are given by

$$
\hat{P}_{r}=\frac{\hbar}{\mathrm{i}}\left(\frac{\partial}{\partial r}+\frac{1}{r}\right), \quad \hat{L}^{2}=-\hbar^{2}\left(\frac{\partial^{2}}{\partial \theta^{2}}+\cot \theta \frac{\partial}{\partial \theta}+\frac{1}{\sin ^{2} \theta} \frac{\partial^{2}}{\partial \phi^{2}}\right), \quad \hat{L}_{z}=\frac{\hbar}{\mathrm{i}} \frac{\partial}{\partial \phi},
$$

in terms of which the spherical Hamiltonian operator can be expressed as

$$
\hat{H}=\frac{1}{2 m} \hat{P}_{r}^{2}+\frac{1}{2 m r^{2}} \hat{L}^{2}+V .
$$

Proof. Substituting $p_{r}=\partial S / \partial r=-\mathrm{i} \hbar \partial(\ln \psi) / \partial r$ from Eqs. (6.2) into (6.6a) yields

$$
P_{r}^{2}=-\frac{\hbar^{2}}{\psi}\left(\frac{2}{r} \frac{\partial \psi}{\partial r}+\frac{\partial^{2} \psi}{\partial r^{2}}\right)=\frac{1}{\psi}\left[\frac{\hbar}{\mathrm{i}}\left(\frac{\partial}{\partial r}+\frac{1}{r}\right)\right]\left[\frac{\hbar}{\mathrm{i}}\left(\frac{\partial}{\partial r}+\frac{1}{r}\right)\right] \psi .
$$

Comparing the above equation to the definition $P_{r}^{2}=(1 / \psi) \hat{P}_{r}^{2} \psi$ gives the desired expression for $\hat{P}_{r}$, the associated operator for the linear momentum in the $r$ direction. Similarly, the expression of Eq. (6.6b) in terms of the wavefunction $\psi$ by using $p_{\theta}=-\mathrm{i} \hbar \partial(\ln \psi) / \partial \theta$ and $p_{\phi}=-\mathrm{i} \hbar \partial(\ln \psi) / \partial \phi$ gives rise to

$$
L^{2}=\frac{-\hbar^{2}}{\psi}\left(\frac{\partial^{2}}{\partial \theta^{2}}+\cot \theta \frac{\partial}{\partial \theta}+\frac{1}{\sin ^{2} \theta} \frac{\partial^{2}}{\partial \phi^{2}}\right) \psi, \quad L_{z}^{2}=\frac{1}{\psi}\left(\frac{\hbar}{\mathrm{i}} \frac{\partial}{\partial \phi}\right)\left(\frac{\hbar}{\mathrm{i}} \frac{\partial}{\partial \phi}\right) \psi,
$$

from which the definitions of $L^{2}=(1 / \psi) \hat{L}^{2} \psi$ and $L_{z}^{2}=(1 / \psi) \hat{L}_{z}^{2} \psi$ yield the expressions for $\hat{L}_{2}$, and $\hat{L}_{z}$. We thus have obtained the operators $\hat{P}_{r}, \hat{L}_{2}$, and $\hat{L}_{z}$ directly from their associated observables defined in quantum Hamilton mechanics without using quantization axiom. In the same way, we can obtain the Hamiltonian operator $\hat{H}$ by writing Eq. (6.3) in the form of $H=(1 / \psi) \hat{H} \psi$ as

$$
\begin{aligned}
H & =-\frac{\hbar^{2}}{2 m} \frac{1}{\psi}\left[\frac{2}{r} \frac{\partial}{\partial r}+\frac{\partial^{2}}{\partial r^{2}}+\frac{1}{r^{2}}\left(\frac{\partial^{2}}{\partial \theta^{2}}+\cot \theta \frac{\partial}{\partial \theta}+\frac{1}{\sin ^{2} \theta} \frac{\partial^{2}}{\partial \phi^{2}}\right)\right] \psi+V \\
& =\frac{1}{\psi}\left(\frac{1}{2 m} \hat{P}_{r}^{2}+\frac{1}{2 m r^{2}} \hat{L}^{2}+V\right) \psi,
\end{aligned}
$$

from which $\hat{H}$ reads as in Eq. (6.8).

It seems that $\hat{H}$ may be obtained by simply applying the quantization axiom to the classical Hamiltonian $H=p_{r}^{2} / 2 m+L^{2} /(2 m r)+V$, but it is noted that $p_{r}$ should be replaced by 
the mechanical momentum operator $\hat{P}_{r}$ in Eq. (6.7) instead of the canonical momentum operator $\hat{p}_{r}=-\mathrm{i} \hbar \partial / \partial r$. This situation is what we had encountered in standard quantum mechanics that the quantization axiom $\mathbf{p} \rightarrow-\mathrm{i} \hbar \nabla$ cannot be applied directly to Spherical coordinates.

In case of central-force field $V=V(r)$, we have $\left[\hat{L}^{2}, \hat{H}\right]=\left[\hat{L}_{z}^{2}, \hat{H}\right]=0$, which implies that the observables $L^{2}$ and $L_{z}^{2}$ are stationary in an eigenstate $\psi$ of $\hat{H}$. From Lemma 3.1, it means that $L^{2}$ and $L_{z}^{2}$ are conservative along any phase-space trajectory $(\mathbf{q}(t), \mathbf{p}(t))$ determined from Eq. (6.4). Since we have already derived the expressions for $L^{2}$ and $L_{z}^{2}$, we can verify their conservation by simply showing $\mathrm{d} L^{2} / \mathrm{d} t=\mathrm{d} L_{z}^{2} / \mathrm{d} t=0$. With the property that the wavefunction $\psi$ has a separable solution $\psi(r, \theta, \phi)=R(r) \Theta(\theta) \Phi(\phi)$ for central-force problem and with the expressions for $H, L^{2}$, and $L_{z}^{2}$ in Eqs. (6.3) and (6.6b), we obtain readily the expected results

$$
\begin{aligned}
& \frac{\mathrm{d} L_{z}^{2}}{\mathrm{~d} t}=\left\{\begin{array}{ll}
L_{z}^{2} & H
\end{array}\right\}=\frac{\partial L_{z}^{2}}{\partial \phi} \frac{\partial H}{\partial p_{\phi}}-\frac{\partial L_{z}^{2}}{\partial p_{\phi}} \frac{\partial H}{\partial \phi}=0, \\
& \frac{\mathrm{d} L^{2}}{\mathrm{~d} t}=\left\{\begin{array}{ll}
L^{2} & H
\end{array}\right\}=\frac{\partial L^{2}}{\partial \theta} \frac{\partial H}{\partial p_{\theta}}-\frac{\partial L^{2}}{\partial p_{\theta}} \frac{\partial H}{\partial \theta}=0 .
\end{aligned}
$$

As Lemma 3.1 indicates, the conserved values for $H, L^{2}$, and $L_{z}^{2}$ in the state $\psi$ are just the eigenvalues of $\hat{H}, \hat{L}_{2}$, and $\hat{L}_{z}^{2}$, respectively. This property is reflected in the relations of $\hat{H} \psi=H \psi, \hat{L}^{2} \psi=L^{2} \psi$, and $\hat{L}_{z}^{2} \psi=L_{z}^{2} \psi$, as expressed in Eqs. (6.10) and (6.9).

Given a quantum state $\psi(r, \theta, \phi)$, we determine its dynamical representation from Eq. (6.4); on the other hand, $\psi^{*} \psi$ also gives us the probability of the particle's spatial distribution. Consequently, there exists close relation between these two different descriptions of $\psi$.

Theorem 6.3. The stable equilibrium radial position predicated from the dynamic representation of $\psi$ in Eq. (6.4) is identical to the position with the maximum radial probability $P(r)=4 \pi r^{2} R^{*}(r) R(r)$ determined from $\psi(r, \theta, \phi)=R(r) \Theta(\theta) \Phi(\phi)$; or stated mathematically,

$$
\frac{\mathrm{d} r}{\mathrm{~d} t}=0 \quad \Longleftrightarrow \quad \frac{\mathrm{d}}{\mathrm{d} r} P(r)=0 .
$$

Proof. Using $\psi(r, \theta, \phi)=R(r) \Theta(\theta) \Phi(\phi)$, we may rewrite Eq. (6.4a) as

$$
\dot{r}=\frac{\hbar}{\mathrm{i} m} \frac{\mathrm{d}}{\mathrm{d} r} \ln (r R(r)) .
$$

Hence, the equilibrium position for $r$ is found from the condition $\dot{r}=\mathrm{d}(\ln (r R)) / \mathrm{d} r=0$, i.e., $\mathrm{d}(r R) / \mathrm{d} r=0$. On the other hand, the radial probability function has the expression $P(r)=R^{*}(r) R(r) 4 \pi r^{2}=4 \pi(r R(r))^{2}$ by noting that $R(r)$ is a real function of $r$ for central-force problems. Accordingly, the maximum radial probability occurs at the location of $\mathrm{d} P / \mathrm{d} r=\mathrm{d}(r R) / \mathrm{d} r=0$, which is just the condition of $\dot{r}=0$.

Example 6.1. For hydrogen atom at ground state, we have $R(r)=\mathrm{e}^{-r / a_{0}}$ with $a_{0}$ being the Bohr radius and $\Theta(\theta)=\Phi(\phi)=1$. Substituting this wavefunction into Eq. (6.4) yields the equations of motion for the ground-state electron as: 


$$
\begin{aligned}
\frac{\mathrm{d} \rho}{\mathrm{d} \tau} & =\frac{2}{\mathrm{i}} \frac{1-\rho}{\rho}, \\
\frac{\mathrm{d} z_{\theta}}{\mathrm{d} \tau} & =\mathrm{i} \frac{z_{\theta}}{\rho^{2}}, \quad z_{\theta}=\cos \theta, \\
\frac{\mathrm{d} \phi}{\mathrm{d} \tau} & =0,
\end{aligned}
$$

where $\rho=r / a_{0}$ is the dimensionless radial distance and $\tau=t \hbar /\left(2 m a_{0}^{2}\right)$ is the dimensionless time. Eq. (6.13a) shows that the ground-state hydrogen atom has an equilibrium radial position $r_{\text {eq }}$ at the Bohr radius $a_{0}$, i.e., $\rho=1$. Meanwhile, the radial probability function is given by $P(r)=4 \pi r^{2} R(r)^{2}=4 \pi r^{2} \mathrm{e}^{-2 r / a_{0}}$ that yields a maximum value at $r_{\max }=a_{0}$ by letting $\mathrm{d} P(r) / \mathrm{d} r=0$. Therefore, we have $r_{\text {eq }}=r_{\max }=a_{0}$.

It is not surprising that the probability of finding a particle at the stable equilibrium position has a maximum value, since there is always a restoring force acting on the particle toward the stable equilibrium position, and once the particle reaches the stable equilibrium position, it will remain there as long as no disturbance is applied. However, Eq. (6.11) alone cannot tell us directly the stability of the equilibrium points. The information of the force action around the equilibrium points is required to judge their stability. The force information is provided by the total potential $V_{\text {Total }}=Q(\psi(\mathbf{q}))+V$ with $Q(\psi(\mathbf{q}))$ being the quantum potential determined from Eq. (1.7). With the help of Eq. (6.4), the Hamiltonian in Eq. (6.3) can be recast into the following form

$$
H=\frac{m}{2}\left[\dot{r}^{2}+(r \dot{\theta})^{2}+(r \dot{\phi} \sin \theta)^{2}\right]+V_{\text {Total }}(r, \theta, \phi)=E,
$$

where the total potential is expressed in terms of the wavefunction $\psi$ as

$$
V_{\text {Total }}=\frac{\hbar^{2}}{8 m r^{2}}\left(4+\cot ^{2} \theta\right)-\frac{\hbar^{2}}{2 m}\left(\frac{\partial^{2} \ln \psi}{\partial r^{2}}+\frac{1}{r^{2}} \frac{\partial^{2} \ln \psi}{\partial \theta^{2}}+\frac{1}{r^{2} \sin ^{2} \theta} \frac{\partial^{2} \ln \psi}{\partial \phi^{2}}\right)+V(r) .
$$

When applied to atomic models, the total potential $V_{\text {Total }}$ exhibits the observed atomic shell structure.

Example 6.2. Considering hydrogen atom, we have wavefunction $\psi_{n l_{l} l}(r, \theta, \phi)=$ $R_{n l}(r) \Theta_{l m_{l}}(\theta) \Phi_{m_{l}}(\phi)$, where $R_{n l}(r)$ is expressed in terms of the Laguerre polynomial $L_{\alpha}^{\beta}(\rho)$ as $R_{n l}(\rho)=(2 \rho / n)^{l} \mathrm{e}^{-\rho / n} L_{n-l-1}^{2 l+1}(2 \rho / n), \rho=r / a_{0}, n \in \mathbf{N} ; \Theta_{l m_{l}}(\theta)$ is expressed in terms of the associated Legendre polynomial $P_{\alpha}^{\beta}(z)$ as $\Theta_{l m_{l}}(\theta)=P_{l}^{m_{l}}(\cos \theta), l=0,1,2, \ldots, n-1$, and $\Phi_{m_{l}}(\phi)=\mathrm{e}^{\mathrm{i} m_{l} \phi}, m_{l}=0, \pm 1, \pm 2, \ldots, \pm l$. The total potential $V_{\text {Total }}$ is state-dependent, and the $V_{\text {Total }}$ relating to $\psi_{n l m_{l}}$ is denoted by $V_{n l m_{l}}$ as

$$
\begin{aligned}
\bar{V}_{n l m_{l}} & =V_{\text {Total }} /\left(\frac{\hbar^{2}}{2 m a_{0}^{2}}\right) \\
& =-\frac{2}{\rho}+\left[\frac{1}{4 \rho^{2}}\left(4+\cot ^{2} \theta\right)-\frac{\mathrm{d}^{2} \ln R_{n l}(\rho)}{\mathrm{d} \rho^{2}}-\frac{1}{\rho^{2}} \frac{\mathrm{d}^{2} \ln \Theta_{l m_{l}}(\theta)}{\mathrm{d} \theta^{2}}\right],
\end{aligned}
$$

where the first term $\bar{V}=-2 / \rho$ is the dimensionless Coulomb potential and the remaining terms in $\bar{V}_{n l m_{l}}$ constitute the quantum potential $\bar{Q}$. The shell structure observed in hydrogen atom is actually caused by $\bar{V}_{n l m_{l}}$, and the quantum force derived from $\bar{V}_{n l m_{l}}$ provides 
the necessary driving force to maintain the electron within the shell. Two typical examples $\bar{V}_{300}$ and $\bar{V}_{310}$ are considered here, which are given by

$$
\begin{aligned}
& \bar{V}_{300}(\rho, \theta)=-\frac{2}{\rho}+\frac{4+\cot ^{2} \theta}{4 \rho^{2}}-\frac{4}{2 \rho^{2}-18 \rho+27}+\frac{(4 \rho-18)^{2}}{\left(2 \rho^{2}-18 \rho+27\right)^{2}}, \\
& \bar{V}_{310}(\rho, \theta)=-\frac{2}{\rho}+\frac{12+4 \tan ^{2} \theta+\cot ^{2} \theta}{4 \rho^{2}}+\frac{1}{(\rho-6)^{2}} .
\end{aligned}
$$

$\bar{V}_{300}$ has three layers distributed in the radial direction and $\bar{V}_{310}$ has four layers with two of them in the radial direction and the other two in the azimuth direction, as shown in Figs. 9 and 10. According to the definition of $\bar{V}_{n l m_{l}}$ in Eq. (6.16), the radial layers are separated by the infinite potential barriers located at the points:

$$
\rho=0 \quad \text { and } \quad R_{n l}(\rho)=(2 \rho / n)^{l} \mathrm{e}^{-\rho / n} L_{n-l-1}^{2 l+1}(2 \rho / n)=0 .
$$

For a given value of $n$, there are $n-l$ different values of $\rho$ satisfying Eq. (6.19), and hence at most $n$ shells can be divided by these values of $\rho$, when $l=0$. In the case of $n=3, l=0$, Eq. (6.19) gives $\rho\left(2 \rho^{2}-18 \rho+27\right)=0$, which can also be obtained directly from the denominator of $\bar{V}_{300}$ in Eq. (6.17). The three layers of $\bar{V}_{300}$ are then located, respectively, in the ranges of $0<\rho<(9-3 \sqrt{3}) / 2,(9-3 \sqrt{3}) / 2<\rho<(9+3 \sqrt{3}) / 2$, and $\rho>(9+3 \sqrt{3}) / 2$, as is shown in Fig. 9 for a 3D surface plot. From probability consideration, the probability that the electron appears at the boundary of the layer is zero, since the radial probability density $P_{n l}(\rho)=4 \pi \rho^{2}\left|R_{n l}(\rho)\right|^{2}$ is identical to zero at the layer boundary determined from Eq. (6.19).

The shell structure of $\bar{V}_{310}$ is distributed in the both radial and azimuth directions. The number and the separation of the azimuth shells are determined by the roots of $\Theta_{l m_{l}}\left(z_{\theta}\right)$. From Eq. (6.16), the potential approaches infinity at the following azimuth angle:

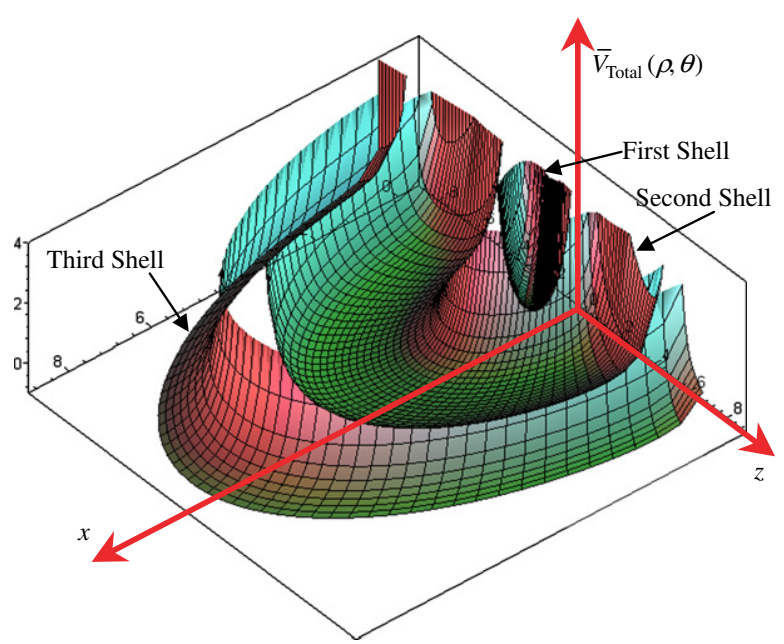

Fig. 9. The triple-shell structure of the total potential $\bar{V}_{\text {Total }}(\rho, \theta)$ for the quantum state $n=3, l=m_{l}=0$, showing that the potential approaches infinity at the shell boundaries where the electron cannot reach and the probability density function $P_{n l}(\rho)=4 \pi \rho^{2}\left|R_{n l}(\rho)\right|^{2}$ is completely zero. The shell boundary locates at the root of $\rho\left(2 \rho^{2}-18 \rho+27\right)=0$, and three layers are then formed, respectively, in the ranges of $0<\rho<(9-3 \sqrt{3}) / 2$, $(9-3 \sqrt{3}) / 2<\rho<(9+3 \sqrt{3}) / 2$, and $\rho>(9+3 \sqrt{3}) / 2$. 


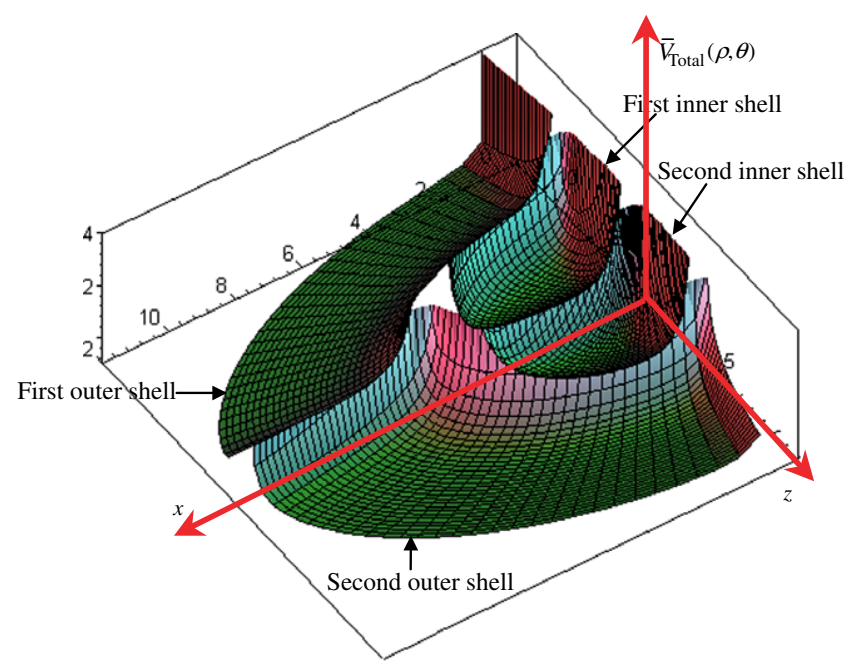

Fig. 10. The quadruple-layer structure of the total potential $\bar{V}_{310}(\rho, \theta)$, showing that the inner and outer layers are respectively, divided into two sub-layers along the azimuth direction with ranges of $0<\theta<\pi / 2$ and $\pi /$ $2<\theta<\pi$. Totally four shells are formed with each containing one equilibrium point: (1) first inner shell occupying the region of $0<\rho<6$, and $0<\theta<\pi / 2$ with $\left(\rho_{\text {eq }}, \theta_{\text {eq }}\right)=\left(3, \cos ^{-1} \sqrt{2 / 3}\right)$, (2) second inner shell occupying the region of $0<\rho<6$, and $\pi / 2<\theta<\pi$ with $\left(\rho_{\text {eq }}, \theta_{\text {eq }}\right)=\left(3, \pi-\cos ^{-1} \sqrt{2 / 3}\right)$, (3) first outer shell occupying the region of $\rho>6$, and $0<\theta<\pi / 2$ with $\left(\rho_{\text {eq }}, \theta_{\text {eq }}\right)=\left(12, \cos ^{-1} \sqrt{2 / 3}\right)$, and (4) second outer shell occupying the region of $\rho>6$, and $\pi / 2<\theta<\pi$ with $\left(\rho_{\mathrm{eq}}, \theta_{\mathrm{eq}}\right)=\left(12, \pi-\cos ^{-1} \sqrt{2 / 3}\right)$.

$$
z_{\theta}=1, \quad \Theta_{l m_{l}}\left(z_{\theta}\right)=\left(1-z_{\theta}^{2}\right)^{\left|m_{l}\right| / 2} \frac{\mathrm{d}^{\left|m_{l}\right|}}{\mathrm{d} z_{\theta}^{\left|m_{l}\right|}} P_{l}\left(z_{\theta}\right)=0,
$$

where $z_{\theta}=\cos \theta$. For a given quantum number $l$, the number of azimuth shells is $l-\left|m_{l}\right|+1$, and the maximum number is $l+1$ in the case of $m_{l}=0$. Accordingly, $\bar{V}_{310}$ has two azimuth shells with boundaries at $z_{\theta}=1$ and $\Theta_{310}\left(z_{\theta}\right)=z_{\theta}=0$, i.e., at $\theta=0$ and $\theta=\pi / 2$. On the other hand, the number of radial shells of $\bar{V}_{310}$ is equal to $n-l=2$ with inner shell in the range of $0<\rho<6$ and outer shell in the range of $\rho>6$, as shown in Fig. 10. Combining together the radial and azimuth shells, we have totally four shells with each containing one equilibrium point: (1) first inner shell occupying the region of $0<\rho<6$, and $0<\theta<\pi / 2$ with $\left(\rho_{\text {eq }}, \theta_{\text {eq }}\right)=\left(3, \cos ^{-1} \sqrt{2 / 3}\right),(2)$ second inner shell occupying the region of $0<\rho<6$, and $\pi / 2<\theta<\pi$ with $\left(\rho_{\text {eq }}, \theta_{\text {eq }}\right)=\left(3, \pi-\cos ^{-1} \sqrt{2 / 3}\right)$, (3) first outer shell occupying the region of $\rho>6$, and $0<\theta<\pi / 2$ with $\left(\rho_{\text {eq }}, \theta_{\text {eq }}\right)=\left(12, \cos ^{-1} \sqrt{2 / 3}\right)$, and (4) second outer shell occupying the region of $\rho>6$, and $\pi / 2<\theta<\pi$ with $\left(\rho_{\text {eq }}, \theta_{\text {eq }}\right)=\left(12, \pi-\cos ^{-1} \sqrt{2 / 3}\right)$. A three-dimensional illustration of the four shells of $\bar{V}_{310}$ is shown in Fig. 10. By evaluating the radial probability density function $P_{n l}(\rho)=4 \pi \rho^{2}\left|R_{n l}(\rho)\right|^{2}$, it can be checked that the maximum value of $P_{n l}(\rho)$ just occurs at the equilibrium points $\left(\rho_{\text {eq }}, \theta_{\text {eq }}\right)$, i.e., the lowermost point in each subshell, as proved in Theorem 6.3.

With given $V_{\text {Total }}$, the quantum forces in the three directions can be determined, respectively, as $f_{r}=-\partial V_{\text {Total }} / \partial r, f_{\theta}=-\partial V_{\text {Total }} / \partial \theta$, and $f_{\phi}=-\partial V_{\text {Total }} / \partial \phi$. These quantum forces together with the Coulomb force establish the stable configurations for atomic structure. 
Example 6.3. Continuing the discussion in Example 6.1, now we are in a position to find the force interaction in the ground state of hydrogen atom and to determine the stability at the position of the Bohr radius. Applying the ground-state wavefunction $\psi_{100}$ to Eq. (6.16) yields

$$
\bar{V}_{\text {Total }}=-\frac{2}{\rho}+\frac{1}{4 \rho^{2}}\left(4+\cot ^{2} \theta\right)
$$

from which the total forces turn out to be

$$
\begin{aligned}
& \bar{f}_{r}=-\frac{\partial \bar{V}_{\text {Total }}}{\partial \rho}=-\frac{2}{\rho^{2}}+\frac{1}{2 \rho^{3}}\left(4+\cot ^{2} \theta\right), \\
& \bar{f}_{\theta}=-\frac{\partial \bar{V}_{\text {Total }}}{\partial \theta}=\frac{1}{2 \rho^{2}} \frac{\cos \theta}{\sin ^{3} \theta}, \quad \bar{f}_{\phi}=-\frac{\partial \bar{V}_{\text {Total }}}{\partial \phi}=0 .
\end{aligned}
$$

The first term $-2 / \rho^{2}$ in Eq. (6.22a) is the dimensionless Coulomb. The total forces $\bar{f}_{r}$ and $\bar{f}_{\theta}$ are a resultant of the electrostatic force and the quantum force. There exists a special position at which the ground-state electron experiences no radial force and tangential force. Letting $\bar{f}_{r}=\bar{f}_{\theta}=0$, we have $\rho_{\text {eq }}=1, \theta_{\text {eq }}=\pi / 2$, which coincides with the equilibrium position determined from $\mathrm{d} \rho / \mathrm{d} \tau=0$ and $\mathrm{d} \theta / \mathrm{d} \tau=0$ in Eq. (6.13). This means that the net force acting on the equilibrium position at the Bohr radius is completely zero. Next, we want to know the stability at the equilibrium point $\rho_{\mathrm{eq}}=1, \theta_{\mathrm{eq}}=\pi / 2$. Evaluating the radial force $\bar{f}_{r}$ at $\theta=\pi / 2$, we obtain

$$
\bar{f}_{r}=\frac{2}{\rho^{3}}-\frac{2}{\rho^{2}},
$$

which shows that the total radial force is the resultant of the repulsive quantum force $-\partial \bar{Q} / \partial \rho=2 / \rho^{3}$ and the attractive Coulomb force $-\partial \bar{V} / \partial \rho=-2 / \rho^{2}$. As $\rho$ approaches zero, the dominative repulsive quantum force increases more rapidly than the attractive Coulomb force, and thus forbids the electron to further approach to the nucleus. In the range of $\rho<1$, i.e., $r<a_{0}$, the repulsive quantum force is greater than the attractive Coulomb force, making the radial distance $\rho$ increase; while in the range of $\rho>1$, the attractive Coulomb force is greater than the repulsive quantum force, making $\rho$ decrease. Hence, there is always a restoring force acting on the electron toward the equilibrium radial location $\rho=1$, indicating that the equilibrium at the Bohr radius is stable.

In the above example, we have shown that the stable configuration of hydrogen ground state is established via the balance between Coulomb force and quantum force. If the attractive Coulomb force were the only force applied on the electron, there would be no additional opposite force to stop the electron from plunging into the nucleus. In quantum states with nonzero magnetic quantum number $m_{l}$, the electron experiences a centripetal force in addition to the Coulomb force and the quantum force $[10,11]$.

Example 6.4. To demonstrate the balance among centripetal force, Coulomb force and quantum force, we consider the quantum state $\psi_{2,1, \pm 1}=\left(\rho \mathrm{e}^{-\rho / 2}\right)(\sin \theta) \mathrm{e}^{ \pm \mathrm{i} \phi}$ with principle quantum number $n=2$, orbital momentum quantum number $l=1$ and magnetic quantum number $m_{l}= \pm 1$. The dynamic representation of $\psi_{2,1, \pm 1}$ is obtained by substituting $\psi_{2,1, \pm 1}$ into Eq. (6.4):

$$
\frac{\mathrm{d} \rho}{\mathrm{d} \tau}=-\frac{1}{\mathrm{i}} \frac{\rho-4}{\rho}, \quad \frac{\mathrm{d} z_{\theta}}{\mathrm{d} \tau}=3 \mathrm{i} \frac{z_{\theta}}{\rho^{2}}, \quad \frac{\mathrm{d} \phi}{\mathrm{d} \tau}=\frac{ \pm 2}{\rho^{2}\left(1-z_{\theta}^{2}\right)},
$$


which indicates that the electron has a nonzero angular rate in the $\phi$ direction. This angular motion will establish different equilibrium condition from those considered in ground state. The radial and tangential forces acting on the electron are computed from the total potential $\bar{V}_{\text {Total }}$ as

$$
\begin{aligned}
& \bar{f}_{r}(\rho, \theta)=-\frac{2}{\rho^{2}}+\frac{1}{2 \rho^{3}}\left(8+\cot ^{2} \theta\right)+\frac{2}{\rho^{3} \sin ^{2} \theta}, \\
& \bar{f}_{\theta}(\rho, \theta)=\frac{5}{2 \rho^{2}} \frac{\cos \theta}{\sin ^{3} \theta} .
\end{aligned}
$$

The equilibrium values for $\rho$ and $\theta$ obtained from $\mathrm{d} \rho / \mathrm{d} \tau=\mathrm{d} z_{\theta} / \mathrm{d} \tau=0$ are $\rho_{\text {eq }}=4$ and $\theta_{\text {eq }}=\pi / 2$. If we evaluate the forces in Eq. (6.25) at the position of $\rho_{\text {eq }}$ and $\theta_{\text {eq }}$, we find $\bar{f}_{r}(4, \pi / 2)=-1 / 32$ and $\bar{f}_{\theta}(4, \pi / 2)=0$, which implies that at the equilibrium position the electron is subjected to a nonzero radial force:

$$
f_{r}=\bar{f}_{r} \frac{\hbar^{2}}{2 m a_{0}^{3}}=-\frac{1}{32} \frac{\hbar^{2}}{2 m a_{0}^{3}} .
$$

At the equilibrium point in Eq. (6.24), we have $\rho(\tau)=4=$ constant, $\theta(\tau)=\pi / 2=$ consatnt, and $\mathrm{d} \phi / \mathrm{d} \tau= \pm 1 / 8$, which implies that the electron has a circular orbit with radius 4 and angular rate $\pm 1 / 8$ in the $x-y$ plane. With this circular orbit in mind, we are naturally led to the conjecture that the net attractive force in Eq. (6.26) must provide the required centripetal force to maintain the stable circular motion in the $x-y$ plane. To verify this point, we have to compute the centripetal force $-m r_{\mathrm{eq}} \dot{\phi}_{\mathrm{eq}}^{2}$ and check whether it equals the radial force in Eq. (6.26). First note that $r_{\text {eq }}=a_{0} \rho_{\text {eq }}=4 a_{0}$ and $\dot{\phi}_{\text {eq }}$ can be calculated from Eq. (6.5c) with $p_{\phi}=-\mathrm{i} \hbar \partial \ln \psi / \partial \phi= \pm \hbar$ :

$$
\dot{\phi}_{\mathrm{eq}}=\frac{p_{\phi}}{m r_{\mathrm{eq}}^{2} \sin ^{2} \theta_{\mathrm{eq}}}= \pm \frac{\hbar}{16 m a_{0}^{2}} .
$$

Then the centripetal force becomes

$$
-m r_{\mathrm{eq}} \dot{\phi}_{\mathrm{eq}}^{2}=-m\left(4 a_{0}\right)\left( \pm \frac{\hbar}{16 m a_{0}^{2}}\right)^{2}=-\frac{1}{32}\left(\frac{\hbar^{2}}{2 m a_{0}^{3}}\right)
$$

This force is identical to the attractive radial force $f_{r}$ obtained from Eq. (6.26).

From quantum mechanics, it is well known that $L^{2}=l(l+1) \hbar^{2}$ and $L_{z}=m_{l} \hbar$ are, respectively, the eigenvalues of $\hat{L}_{2}$ and $\hat{L}_{z}$ associated with the eigenfunction $\psi_{n, l, m_{l}}$; whereas according to Lemma $3.1, L^{2}=l(l+1) \hbar^{2}$ and $L_{z}=m_{l} \hbar$ must be two motion constants along any trajectory in the dynamic system representing $\psi_{n, l, m_{l}}$. The following example illustrates the conservation of $L^{2}$ and $L_{z}$ along quantum trajectory.

Example 6.5. Consider the quantum state $\psi_{2,1, \pm 1}=\left(\rho \mathrm{e}^{-\rho / 2}\right)(\sin \theta) \mathrm{e}^{ \pm \mathrm{i} \phi}$ whose dynamic representation has been given in Eq. (6.24). We want to show that along any trajectory determined from Eq. (6.24), the two observables $L^{2}(t)$ and $L_{z}(t)$ are conserved at the values of $l(l+1) \hbar^{2}=2 \hbar^{2}$ and $m_{l} \hbar= \pm \hbar$, respectively. Inserting $\partial \ln \psi / \partial \phi=\mathrm{d} \ln \Phi / \mathrm{d} \phi= \pm \mathrm{i}$ into Eq. (6.5c), we obtain

$$
L_{z}(t)=m r^{2} \dot{\phi} \sin ^{2} \theta=p_{\phi}=\frac{\hbar}{\mathrm{i}} \frac{\partial \ln \psi}{\partial \phi}= \pm \hbar=\text { constant }
$$


which not only ensures the conservation of $L_{z}$ but also identifies the conserved value of $L_{z}$ as $\pm \hbar$. In conjunction with Eq. (6.4), the orbital angular momentum $L^{2}$ derived in Eq. (6.6b) can be rewritten as

$$
\begin{aligned}
L^{2}(t) & =\left(m r^{2} \dot{\theta}\right)^{2}+\left(m r^{2} \dot{\phi} \sin \theta\right)^{2}+\frac{\hbar^{2}}{4} \cot ^{2} \theta-\hbar^{2} \frac{\mathrm{d}^{2} \ln \Theta(\theta)}{\mathrm{d} \theta^{2}} \\
& =\frac{\hbar^{2}}{4}\left[\left(\rho^{2} \frac{\mathrm{d} \theta}{\mathrm{d} \tau}\right)^{2}+\left(\rho^{2} \frac{\mathrm{d} \phi}{\mathrm{d} \tau} \sin \theta\right)^{2}+\cot ^{2} \theta-4 \frac{\mathrm{d}^{2} \ln \Theta(\theta)}{\mathrm{d} \theta^{2}}\right] .
\end{aligned}
$$

Now we evaluate $L^{2}$ by using $\mathrm{d} \theta / \mathrm{d} \tau$ and $\mathrm{d} \phi / \mathrm{d} \tau$ from Eq. (6.24) and $\Theta_{1, \pm 1}(\theta)=\sin \theta$ to see whether $L^{2}$ is a constant. This evaluation leads to

$$
L^{2}(t)=\frac{\hbar^{2}}{4}\left[(-3 i \cot \theta)^{2}+\left(\frac{ \pm 2}{\sin \theta}\right)^{2}+\cot ^{2} \theta-4\left(-\csc ^{2} \theta\right)\right]=2 \hbar^{2} .
$$

Hence $L^{2}$ is truly a constant of motion in the state of $n=2, l=1$, and $m_{l}= \pm 1$. The constant value of $L^{2}$ obtained from Eq. (6.31) is the same as the eigenvalue $l(l+1) \hbar^{2}$ of the quantum operator $\hat{L}_{2}$ evaluated at $l=1$. It is noted that $\rho(\tau), \theta(\tau)$, and $\phi(\tau)$ computed from Eq. (6.24) are, in general, complex functions, however, the orbital angular momentum $L^{2}$ formed by them using Eq. (6.30) is always real. This property is attributed to the Hermiticity of $\hat{L}_{2}$.

Before leaving this section, we apply quantum Hamilton mechanics to give a formal proof of Sommerfeld-Wilson quantization rule regarding hydrogen atom.

Theorem 6.4. Let $(r, \theta, \phi)$ and $\left(p_{r}, p_{\theta}, p_{\phi}\right)$ be canonical variables describing the motion of the electron in hydrogen atom, and let $c$ be any closed trajectory traced by the electron. Then the action variables $\Phi_{c} p_{r} \mathrm{~d} r, \Phi_{c} p_{\theta} \mathrm{d} \theta$, and $\Phi_{c} p_{\phi} \mathrm{d} \phi$ must be equal to some integral multiples of $h$.

Proof. Action variables are the contour integrals of the canonical momenta along a closed trajectory $c$ traced by the electron in complex plane. The canonical momenta can be found by inserting $\psi(r, \theta, \phi)=R_{n l}(r) \Theta_{l m_{l}}(\theta) \Phi_{m_{l}}(\phi)$ in Eq. (6.2):

$$
p_{r}=\frac{\hbar}{\mathrm{i}} \frac{\mathrm{d} \ln R_{n l}(r)}{\mathrm{d} r}, \quad p_{\theta}=\frac{\hbar}{\mathrm{i}} \frac{\mathrm{d} \ln \Theta_{l m_{l}}(\theta)}{\mathrm{d} \theta}, \quad p_{\phi}=\frac{\hbar}{\mathrm{i}} \frac{\mathrm{d} \ln \Phi_{m_{l}}(\phi)}{\mathrm{d} \phi} .
$$

Using $\Phi_{m_{l}}(\phi)=\mathrm{e}^{\mathrm{i} m_{l} \phi}$ in the evaluation of $p_{\phi}$, we obtain readily the quantization rule in the $\phi$ direction

$$
\oint_{c} p_{\phi} \mathrm{d} \phi=\frac{\hbar}{\mathrm{i}} \oint_{c}\left(i m_{l}\right) \mathrm{d} \phi=\hbar m_{l}(2 \pi)=m_{l} h, \quad\left|m_{l}\right|=0,1, \cdots, l-1 .
$$

Next, we consider the quantization in the $r$ direction. The use of $R_{n l}(\rho)=(2 \rho / n)^{l}$ $\mathrm{e}^{-\rho / n} L_{n-l-1}^{2 l+1}(2 \rho / n)$ in $p_{r}$ leads to

$$
\oint_{c} p_{r} \mathrm{~d} r=\frac{\hbar}{\mathrm{i}} \oint_{c} \frac{R_{n l}^{\prime}(\rho)}{R_{n l}(\rho)} \mathrm{d} \rho=\frac{h}{2 \pi \mathrm{i}} \oint_{c} \frac{F_{n l}^{\prime}(\rho)}{F_{n l}(\rho)} \mathrm{d} \rho
$$

where $F_{n l}(\rho)=\rho^{l} L_{n-l-1}^{2 l+1}(\rho)$ is a $(n-1)$ th-order polynomial. From the residue theorem as stated in Eq. (4.14), the value of the contour integration in Eq. (6.34) depends only on $n_{r}$, the number of roots of $F_{n l}(\rho)$ encircled by the contour $c$, 


$$
\oint_{c} p_{r} \mathrm{~d} r=\frac{h}{2 \pi \mathrm{i}} \oint_{c} \frac{F_{n l}^{\prime}(\rho)}{F_{n l}(\rho)} \mathrm{d} \rho=n_{r} h, \quad n_{r}=0,1, \cdots, n-1 .
$$

The maximum value of $n_{r}$ is $n-1$, since the order of the polynomial $F_{n l}(\rho)$ is $n-1$; the minimum value of $n_{r}$ is zero, when the contour $c$ encloses no root of $F_{n l}(\rho)$. Eq. (6.35) confirms the Sommerfeld-Wilson quantization in the radial direction. As for the evaluation of $\oint p_{\theta} \mathrm{d} \theta$, the substitution of $\Theta_{l m_{l}}(\theta)=P_{l}^{m_{l}}(\cos \theta)$ into $p_{\theta}$ gives

$$
\oint_{c} p_{\theta} \mathrm{d} \theta=\frac{\hbar}{\mathrm{i}} \oint_{c} \frac{\Theta_{l m_{l}}^{\prime}(\theta)}{\Theta_{l m_{l}}(\theta)} \mathrm{d} \theta=\frac{h}{2 \pi \mathrm{i}} \oint_{c} \frac{\Theta_{l m_{l}}^{\prime}\left(z_{\theta}\right)}{\Theta_{l m_{l}}\left(z_{\theta}\right)} \mathrm{d} z_{\theta} .
$$

To facilitate the contour integration, $\Theta_{l m_{l}}\left(z_{\theta}\right)$ is further expressed in terms of the Legendre polynomial $P_{l}\left(z_{\theta}\right)$, leading to

$$
\begin{aligned}
\oint_{c} p_{\theta} \mathrm{d} \theta & =\frac{\left|m_{l}\right| h}{2 \pi \mathrm{i}} \oint_{c} \frac{z_{\theta}}{z_{\theta}^{2}-1} \mathrm{~d} z_{\theta}+\frac{h}{2 \pi \mathrm{i}} \oint_{c} \frac{\mathrm{d}^{\left|m_{l}\right|+1} P_{l}\left(z_{\theta}\right) / \mathrm{d} z_{\theta}^{\left|m_{l}\right|+1}}{\mathrm{~d}^{\left|m_{l}\right|} P_{l}\left(z_{\theta}\right) / \mathrm{d} z_{\theta}^{\left|m_{l}\right|}} \mathrm{d} z_{\theta}, \\
& =\left|m_{l}\right| h+n_{\theta}^{\prime} h=n_{\theta} h, \quad n_{\theta}=0,1, \cdots, l
\end{aligned}
$$

where $n_{\theta}^{\prime}$ is the number of roots of $\mathrm{d}^{\left|m_{l}\right|} P_{l}\left(z_{\theta}\right) / \mathrm{d} z_{\theta}^{\left|m_{l}\right|}$ enclosed by the contour $c$. Since $\mathrm{d}^{\left|m_{l}\right|} P_{l}\left(z_{\theta}\right) / \mathrm{d} z_{\theta}^{\left|m_{l}\right|}$ is a polynomial of order $l-\left|m_{l}\right|$, the maximum value of $n_{\theta}^{\prime}$ is $l-\left|m_{l}\right|$, hence the maximum value of $n_{\theta}=\left|m_{l}\right|+n_{\theta}^{\prime}$ becomes $l$.

\section{Observables and operators in curvilinear coordinates}

Having derived quantum operators in spherical coordinates, we proceed to consider quantum Hamilton mechanics under general curvilinear coordinates, for which we shall need tensor representation of the Hamiltonian $H$. Consider an $n$-dimensional space $\mathbf{V}_{n}$ covered by a curvilinear coordinate system $\mathbf{q}$. We metrize $\mathbf{V}_{n}$ by prescribing the elements of $\operatorname{arcd} s$, so that

$$
\mathrm{d} s^{2}=g_{j k} \mathrm{~d} q^{j} \mathrm{~d} q^{k}
$$

is a positive definite quadratic form in the differentials $\mathrm{d} q^{j}$. In Eq. (7.1), we have used the summation convention that if in some expression, a certain index occurs twice with one in the subscript and the other in the superscript, we shall mean that this expression is summed with respect to that index for all admissible values of the index. The $C^{1}$ functions $g_{j k}(\mathbf{q})$ are the elements of the metric tensor, which completely determine the essential metric properties of $\mathbf{V}_{n}$. The space $\mathbf{V}_{n}$ so metrized is an $n$-dimensional Riemannian space $\mathbf{R}_{n}$, wherein we need to distinct the contravariant coordinates $q^{j}, j=1,2, \ldots, n$ from the covariant coordinates $q_{j}, j=1,2, \ldots, n$ by noting the relation

$$
q_{j}=g_{j k} q^{k} \quad \text { or } \quad q^{j}=g^{j k} q_{k}
$$

where $g^{j k}$ are the elements of the inverse of the matrix $\left[g_{j k}\right]$, i.e., $\left[g^{j k}\right]=\left[g_{j k}\right]^{-1}$. Similarly, the contravariant momentum $p^{j}$ relates to the covariant momentum $p_{j}$ as:

$$
p_{j}=g_{j k} p^{k} \quad \text { or } \quad p^{j}=g^{j k} p_{k} .
$$

If Cartesian coordinates are concerned, we have $g_{j k}=\delta_{j k}$ and thus contravariant and covariant components become identical so that $q^{j}=q_{j}$ and $p^{j}=p_{j}$. The quantum Hamiltonian in Eq. (1.6) represented in the Euclidean space can be extended to the Riemannian 
space $\mathbf{R}_{n}$ by tensor manipulations. First, consider the kinetic energy $\mathbf{p} \cdot \mathbf{p} /(2 m)$ in Eq. (1.6), which can be expressed in $\mathbf{R}_{n}$ as

$$
K=\frac{1}{2 m} \mathbf{p} \cdot \mathbf{p}=\frac{1}{2 m} p^{j} p_{j}=\frac{1}{2 m} g^{j k} p_{k} p_{j}
$$

where the relation (7.3) has been used. The complex potential $Q=\hbar /(2 m \mathrm{i}) \nabla \cdot \mathbf{p}$ in Eq. (1.7) is defined in terms of the divergence of momentum $\mathbf{p}$, which, when expressed in tensor form, becomes

$$
Q(\mathbf{q}, t)=\frac{\hbar}{2 m i} \partial_{j} p^{j}=\frac{\hbar}{2 m i}\left(\frac{\partial p^{j}}{\partial q^{j}}+\left\{\begin{array}{c}
j \\
k j
\end{array}\right\} p^{k}\right),
$$

where $\partial_{j} p^{j}$ denotes the covariant derivative of the contravariant tensor $p^{j}$, and the second kind Christoffel symbol is defined as

$$
\left\{\begin{array}{c}
l \\
k j
\end{array}\right\}=g^{l \alpha}[k j, \alpha]=\frac{1}{2} g^{l \alpha}\left(\frac{\partial g_{k \alpha}}{\partial q^{j}}+\frac{\partial g_{j \alpha}}{\partial q^{k}}-\frac{\partial g_{k j}}{\partial q^{\alpha}}\right) .
$$

Letting $l=j$ in Eq. (7.6), it can be shown

$$
\left\{\begin{array}{c}
j \\
k j
\end{array}\right\}=\frac{\partial}{\partial q^{k}} \ln \sqrt{g}
$$

with $g$ being the determinant of the matrix $\left[g_{j k}\right]$. Hence, the quantum potential expressed in tensor form becomes

$$
\begin{aligned}
Q & =\frac{\hbar}{2 m \mathrm{i}} \partial_{j} p^{j}=\frac{\hbar}{2 m \mathrm{i}}\left(\frac{\partial p^{j}}{\partial q^{j}}+p^{k} \frac{\partial \ln \sqrt{g}}{\partial q^{k}}\right)=\frac{\hbar}{2 m \mathrm{i}} \frac{1}{\sqrt{g}} \frac{\partial}{\partial q^{j}}\left(\sqrt{g} p^{j}\right) \\
& =\frac{\hbar}{2 m \mathrm{i}} \frac{1}{\sqrt{g}} \frac{\partial}{\partial q^{j}}\left(\sqrt{g} g^{j k} p_{k}\right) .
\end{aligned}
$$

For a given coordinate system, $g$ and $g^{j k}$ are known functions of the generalized coordinate $q^{j}$. In a given quantum state $\psi(\mathbf{q}, t)$, the canonical momentum $p_{j}$ can be determined as in Eq. (1.8)

$$
p_{j}=\frac{\partial S(\mathbf{q}, t)}{\partial q^{j}}=\partial_{j} S=-\mathrm{i} \hbar \partial_{j} \ln \psi(\mathbf{q}, t) .
$$

It is worthy of noting that in Eq. (7.9) $p_{j}=\partial_{j} S$ denotes the covariant derivative of $S$, which is different from the gradient $\nabla S$ (the contravariant derivative of $S$ ) whose components are represented by

$$
\partial^{j} S=g^{j k} \partial_{k} S
$$

$\partial_{j} S$ and $\partial^{j} S$ are equal only under Cartesian coordinates. Accordingly, the expression $\mathbf{p}=\nabla S$ is merely applicable to Cartesian coordinates, while general expression for the conjugate momentum p must be in the form of Eq. (7.9). Using Eq. (7.9), the quantum potential in Eq. (7.8) turns out to be

$$
Q=\frac{\hbar}{2 m \mathrm{i}} \frac{1}{\sqrt{g}} \frac{\partial}{\partial q^{j}}\left(\sqrt{g} g^{j k} p_{k}\right)=\frac{\hbar}{2 m \mathrm{i}}\left[\frac{\hbar}{\mathrm{i}} g^{j k} \frac{\partial^{2} \ln \psi}{\partial q^{j} \partial q^{k}}+\frac{p_{k}}{\sqrt{g}} \frac{\partial}{\partial q^{j}}\left(\sqrt{g} g^{j k}\right)\right] .
$$


Now the quantum Hamiltonian defined in Eq. (1.6) under general curvilinear coordinates $q^{j}$ can be constructed as

$$
\begin{aligned}
H & =\frac{1}{2 m} g^{j k} p_{k} p_{j}+V(\mathbf{q})+\frac{\hbar}{2 m \mathrm{i}} \frac{1}{\sqrt{g}} \frac{\partial}{\partial q^{j}}\left(\sqrt{g} g^{j k} p_{k}\right) \\
& =\frac{1}{2 m} g^{j k} p_{k} p_{j}+V(\mathbf{q})-\frac{\hbar^{2}}{2 m} g^{j k} \frac{\partial^{2} \ln \psi}{\partial q^{j} \partial q^{k}}+\frac{\hbar}{2 m \mathrm{i}} \frac{p_{k}}{\sqrt{g}} \frac{\partial}{\partial q^{j}}\left(\sqrt{g} g^{j k}\right),
\end{aligned}
$$

where, as noted previously, the quantum Hamiltonian $H$ is state-dependent; different state $\psi$ produces different Hamiltonian $H$. The Hamilton equations of motion accompanying the quantum Hamiltonian (7.12b) read

$$
\begin{aligned}
\frac{\mathrm{d} q^{k}}{\mathrm{~d} t}= & \frac{\partial H}{\partial p_{k}}=\frac{1}{m} g^{j k} p_{j}+\frac{\hbar}{2 m \mathrm{i}} \frac{1}{\sqrt{g}} \frac{\partial}{\partial q^{j}}\left(\sqrt{g} g^{j k}\right), \\
\frac{\mathrm{d} p_{k}}{\mathrm{~d} t}= & -\frac{\partial H}{\partial q^{k}}=-\frac{p_{j} p_{n}}{2 m} \frac{\partial g^{j n}}{\partial q^{k}}-\frac{\partial}{\partial q^{k}}\left[V(\mathbf{q})-\frac{\hbar^{2}}{2 m} g^{j k} \frac{\partial^{2} \ln \psi}{\partial q^{j} \partial q^{k}}\right] \\
& -\frac{\hbar}{2 m \mathrm{i}} \frac{p_{n}}{\sqrt{g}} \frac{\partial}{\partial q^{k} \partial q^{j}}\left(\sqrt{g} g^{j n}\right) .
\end{aligned}
$$

As in classical Hamilton mechanics, when deriving the Hamilton equations (7.13) from the Hamiltonian (7.12b), the canonical variables $p_{k}$ and $q^{k}$ are regarded as independent variables. Two special cases of Eq. (7.13) have been considered in the previous sections. In Cartesian coordinates, we have $g^{j k}=g_{j k}=\delta_{j k}$ and $g=1$, and then Eq. (7.13) reduces to Eq. (1.9) automatically. In spherical coordinates, we have $g_{11}=1 / g^{11}=1, g_{22}=$ $1 / g^{22}=r^{2}, g_{33}=1 / g^{33}=r^{2} \sin ^{2} \theta, g=g_{11} g_{22} g_{33}=r^{4} \sin ^{2} \theta$, and $g_{j k}=g^{j k}=0$ for $j \neq k$. With the above substitutions, Eq. (7.13a) becomes Eq. (6.5). Solving the quantum Hamilton equations (7.13) is tantamount to solving the Schrödinger equation, and their relationship can be expressed as in the following theorem.

Theorem 7.1. $p_{k}=\partial S / \partial q^{k}=-\mathrm{i} \hbar \partial \ln \psi / \partial q^{k}$ is a solution of Eq. (7.13b), if $S$ is a solution of the quantum Hamilton-Jacobi equation, or equivalently, if $\psi$ is a solution of the Schrödinger equation.

Proof. Given $p_{k}=\partial S / \partial q^{k}$, we can express $\dot{p}_{k}$ in Eq. (7.13b) as

$$
\frac{\mathrm{d} p_{k}}{\mathrm{~d} t}=\frac{\partial p_{k}}{\partial t}+\frac{\partial p_{k}}{\partial q^{j}} \dot{q}^{j}=\frac{\partial^{2} S}{\partial t \partial q^{k}}+\frac{\partial p_{j}}{\partial q^{k}} \frac{\partial H}{\partial p_{j}}=-\frac{\partial H}{\partial q^{k}},
$$

where we have made use of Eq. (7.13a) and the identity in Eq. (2.4). The above equation can be rewritten in the form of total differentiation with respect to $q^{k}$ :

$$
\frac{\mathrm{d}}{\mathrm{d} q^{k}}\left(\frac{\partial S}{\partial t}+H\left(t, q^{k}, p_{j}\left(q^{k}\right)\right)\right)=0
$$

where the total differentiation stands for $\mathrm{d} / \mathrm{d} q^{k}=\partial / \partial q^{k}+\left(\partial p_{j} / \partial q^{k}\right) \partial / \partial p_{j}$, and since $S$ is not a function of $p_{j}$, we have $\mathrm{d} S / \mathrm{d} q^{k}=\partial S / \partial q^{k}$. Eq. (7.14) is satisfied, if $S$ is a solution of the following quantum Hamilton-Jacobi equation:

$$
\frac{\partial S}{\partial t}+\left.H\left(t, q^{k}, p_{j}\left(q^{k}\right)\right)\right|_{p_{j}=\partial S / \partial q^{j}}=0
$$


which can be expressed explicitly, by inserting $p_{j}=\partial S / \partial q^{j}$ in Eq. (7.12a), as

$$
\frac{\partial S}{\partial t}+\frac{g^{j k}}{2 m} \frac{\partial S}{\partial q^{j}} \frac{\partial S}{\partial q^{k}}+V+\frac{\hbar}{2 m \mathrm{i}} \frac{1}{\sqrt{g}} \frac{\partial}{\partial q^{j}}\left(\sqrt{g} g^{j k} \frac{\partial S}{\partial q^{k}}\right)=0 .
$$

The change of variable $S=-\mathrm{i} \hbar \ln \psi$ then transforms the above quantum Hamilton-Jacobi equation to the equation

$$
\mathrm{i} \hbar \frac{\partial \psi}{\partial t}=-\frac{\hbar^{2}}{2 m} \partial_{k} \partial^{k} \psi+V \psi
$$

where $\partial_{k} \partial^{k} \psi$ denotes the Laplacian of $\psi$ in the curvilinear coordinates $q^{j}$ defined as

$$
\partial_{k} \partial^{k} \psi=g^{k j} \partial_{k} \partial_{j} \hbar \nabla^{2} \psi=\frac{1}{\sqrt{g}} \frac{\partial}{\partial q^{j}}\left(\sqrt{g} g^{j k} \frac{\partial \psi}{\partial q^{k}}\right) .
$$

The Hamiltonian operator $\hat{H}$ corresponding to the Hamiltonian $H$ in Eq. (7.12a) can be found by expressing $H=(1 / \psi) \hat{H} \psi$ in the form of Eq. (3.1). The substitution of Eq. (7.9) into Eq. (7.12a) leads to

$$
H=\frac{1}{2 m}\left(\frac{\hbar}{\mathrm{i}} \frac{1}{\psi}\right)^{2} g^{j k} \frac{\partial \psi}{\partial q^{j}} \frac{\partial \psi}{\partial q^{k}}+V+\frac{1}{2 m}\left(\frac{\hbar}{\mathrm{i}}\right)^{2} \frac{1}{\sqrt{g}} \frac{\partial}{\partial q^{j}}\left(\frac{1}{\psi} \sqrt{g} g^{j k} \frac{\partial \psi}{\partial q^{k}}\right) .
$$

The recombination of the first and third terms in the right-hand side results in

$$
H=\frac{1}{\psi}\left(-\frac{\hbar^{2}}{2 m} \frac{1}{\sqrt{g}} \frac{\partial}{\partial q^{j}} \sqrt{g} g^{j k} \frac{\partial}{\partial q^{k}}+V\right) \psi=\frac{1}{\psi}\left(-\frac{\hbar^{2}}{2 m} \partial_{k} \partial^{k}+V\right) \psi,
$$

from which $\hat{H}$ can be identified as

$$
\hat{H}=-\frac{\hbar^{2}}{2 m} \partial_{k} \partial^{k}+V,
$$

and the Schrödinger equation (7.17) becomes the familiar form

$$
\mathrm{i} \hbar \frac{\partial \psi}{\partial t}=\hat{H} \psi \text {. }
$$

In this way, we have derived a general expression for $\hat{H}$ directly in the curvilinear coordinates $q^{j}$ without transforming back to Cartesian coordinates by using quantization axiom. Next, we consider the moment operator $\hat{p}_{j}$ in the curvilinear coordinates $q^{j}$.

Lemma 7.1. In any curvilinear coordinates $q^{j}$, the canonical operator $\hat{p}_{j}$ associated with the covariant momentum $p_{j}$ is always given by

$$
\hat{p}_{j}=-\mathrm{i} \hbar \frac{\partial}{\partial q^{j}} .
$$

Proof. From Eq. (7.9), we have $p_{j}=(1 / \psi)\left(-\mathrm{i} \hbar \partial / \partial q^{j}\right) \psi$ which, when compared with the definition $p_{j}=(1 / \psi) \hat{p}_{j} \psi$, gives $\hat{p}_{j}=-\mathrm{i} \hbar \partial / \partial q^{j}$.

The canonical momentum $p_{j}$ may not be identical to the mechanical momentum $P_{j}$ with which we are concerned. To find the mechanical momentum operator $\hat{P}_{j}$ in the curvilinear coordinates $q^{j}$, we need the detailed information regarding the metric tensor $g_{j k}$. In this section, we shall focus on the orthogonal curvilinear coordinates that we often encounter in 
quantum mechanics. General curvilinear coordinates will be considered in the next section. In orthogonal coordinate systems, we have

$$
g_{j k}= \begin{cases}h_{j}^{2} & j=k, \\ 0 & j \neq k .\end{cases}
$$

Let $\mathbf{r}\left(q^{k}\right)$ be the position vector of a particle whose velocity then can be expressed by

$$
\dot{\mathbf{r}}=\dot{q}^{k} \frac{\partial \mathbf{r}}{\partial q^{k}}=\dot{q}^{k} \mathbf{a}_{k}=\left(\dot{q}^{k} h_{k}\right) \mathbf{e}_{k},
$$

where $\dot{q}^{k}$ is known as the contravariant velocity and the tangential vector $\mathbf{a}_{k}$ connects to the element of metric tensor $g_{j k}$ as

$$
g_{j k}=\frac{\partial \mathbf{r}}{\partial q^{j}} \cdot \frac{\partial \mathbf{r}}{\partial q^{k}}=\mathbf{a}_{j} \cdot \mathbf{a}_{k}
$$

The scale factor $h_{k}=\left|\mathbf{a}_{k}\right|$ is the magnitude of $\mathbf{a}_{k}$ and $\mathbf{e}_{k}=\mathbf{a}_{k} /\left|\mathbf{a}_{k}\right|=\mathbf{a}_{k} / h_{k}$ is the unit vector along the direction of $\mathbf{a}_{k}$. The contravariant mechanical momentum is $P^{j}=m \dot{q}^{j}$, from which the covariant component $P_{j}$ turns into

$$
P_{j}=g_{j k} P^{k}=m g_{j k} \dot{q}^{k} .
$$

The associated operator $\hat{P}_{j}$ now can be established as in the following theorem.

Theorem 7.2. In the orthogonal curvilinear coordinates $q^{j}$ with scale factor $h_{j}$, the mechanical momentum operator $\hat{P}_{j}$ is given by

$$
\hat{P}_{j}=\hat{p}_{j}+\frac{\hbar}{2 \mathrm{i}} \frac{\partial}{\partial q^{j}} \ln \frac{h_{1} h_{2} h_{3}}{h_{j}^{2}}, \quad j=1,2,3 .
$$

Proof. To find $\hat{P}_{j}$, we have to derive the expressions for $P_{j}$ from Eq. (7.25), where $\dot{q}^{j}$ comes from the Hamilton equation of motion (7.13a) with $g_{j k}$ given by Eq. (7.22):

$$
\dot{q}^{j}=\frac{p_{j}}{m h_{j}^{2}}+\frac{1}{2 m \mathrm{i}} \frac{\hbar}{h_{1} h_{2} h_{2}} \frac{\partial}{\partial q^{j}} \frac{h_{1} h_{2} h_{2}}{h_{j}^{2}} .
$$

Substituting $\dot{q}^{j}$ into Eq. (7.25) and using $p_{j}=-\mathrm{i} \hbar \partial \ln \psi / \partial q^{j}$, we obtain

$$
\begin{aligned}
P_{j} & =m g_{j k} \dot{q}^{k}=m g_{j k}\left[\frac{p_{k}}{m h_{k}^{2}}+\frac{1}{2 m \mathrm{i}} \frac{\hbar}{h_{1} h_{2} h_{2}} \frac{\partial}{\partial q^{k}} \frac{h_{1} h_{2} h_{2}}{h_{k}^{2}}\right]=p_{j}+\frac{\hbar}{2 \mathrm{i}} \frac{h_{j}^{2}}{h_{1} h_{2} h_{3}} \frac{\partial}{\partial q^{j}} \frac{h_{1} h_{2} h_{3}}{h_{j}^{2}} \\
& =\frac{1}{\psi}\left[\frac{\hbar}{\mathrm{i}} \frac{\partial}{\partial q^{j}}+\frac{\hbar}{2 \mathrm{i}} \frac{\partial}{\partial q^{j}} \ln \frac{h_{1} h_{2} h_{3}}{h_{j}^{2}}\right] \psi,
\end{aligned}
$$

where we have employed the orthogonal property of $g_{j k}$ defined in Eq. (7.22). The comparison to the definition $P_{j}=(1 / \psi) \hat{P}_{j} \psi$ then yields the expression for $\hat{P}_{j}$ in Eq. (7.26).

A remarkable consequence of Theorem 7.2 is that only in Cartesian coordinates is $\hat{P}_{j}$ equal to $\hat{p}_{j}$ because of $h_{1}=h_{2}=h_{3}=1$ in Eq. (7.26). In curvilinear coordinates, we have $h_{j} \neq 1$ and thus $\hat{P}_{j} \neq \hat{p}_{j}=-\mathrm{i} \hbar \partial / \partial q^{j}$, which explains why the quantization principle $\mathbf{p} \rightarrow \hat{\mathbf{p}}=-\mathrm{i} \hbar \nabla$ is only valid for Cartesian coordinates. Theorem 7.2 allows us to determine $\hat{P}_{j}$ directly in any orthogonal curvilinear coordinates. 
Example 7.1. Applying Theorem 7.2 to spherical coordinates, where $q^{1}=r, q^{2}=\theta$, $q^{3}=\phi, h_{1}=1, h_{2}=r$, and $h_{3}=r \sin \theta$, we have

$$
\begin{aligned}
& \frac{\partial}{\partial q^{1}} \ln \frac{h_{1} h_{2} h_{3}}{h_{1}^{2}}=\frac{\partial}{\partial r} \ln \frac{r^{2} \sin \theta}{1}=\frac{2}{r}, \\
& \frac{\partial}{\partial q^{2}} \ln \frac{h_{1} h_{2} h_{3}}{h_{2}^{2}}=\frac{\partial}{\partial \theta} \ln \frac{r^{2} \sin \theta}{r^{2}}=\cot \theta, \\
& \frac{\partial}{\partial q^{3}} \ln \frac{h_{1} h_{2} h_{3}}{h_{3}^{2}}=\frac{\partial}{\partial \phi} \ln \frac{r^{2} \sin \theta}{r^{2} \sin ^{2} \theta}=0 .
\end{aligned}
$$

Then Eq. (7.26) leads to

$$
\hat{P}_{r}=\frac{\hbar}{\mathrm{i}}\left(\frac{\partial}{\partial r}+\frac{1}{r}\right), \quad \hat{P}_{\theta}=\frac{\hbar}{\mathrm{i}}\left(\frac{\partial}{\partial \theta}+\frac{\cot \theta}{2}\right), \quad \hat{P}_{\phi}=\frac{\hbar}{\mathrm{i}} \frac{\partial}{\partial \phi} .
$$

It can be seen that $\hat{P}_{r}$ and $\hat{P}_{\phi}=\hat{L}_{z}$ are identical to those derived in Eq. (6.7), where they have been obtained alternatively from the expressions for $P_{r}$ and $p_{\phi}$. Here, we obtain the same $\hat{P}_{r}$ and $\hat{P}_{\phi}$ directly from the Hamilton equations of motion (7.27). Note that $\hat{P}_{r}$ and $\hat{P}_{\theta}$ are distinct from the canonical momentum operators $\hat{p}_{r}$ and $\hat{p}_{\theta}$ in the additional terms $-\mathrm{i} \hbar / r$ and $(-\mathrm{i} \hbar / 2) \cot \theta$, which arise from the nonconstant scalar factors $h_{j}$ in spherical coordinates.

It is well known that the angular momentum operator $\hat{L}_{2}$ defined in Eq. (6.7) involves only orbit motion. The momentum operator $\hat{P}_{\theta}$ derived in Eq. (7.28) contains spin effect and relates to the total angular momentum. The corresponding local angular momentum $P_{\theta}$ can be found from the relation (3.1) as

$$
P_{\theta}=\frac{1}{\psi} \hat{P}_{\theta} \psi=\frac{\hbar}{\mathrm{i}} \frac{1}{\psi} \frac{\partial \psi}{\partial \theta}+\frac{\hbar}{\mathrm{i}} \frac{\cot \theta}{2}=L_{\theta}+S,
$$

where $L_{\theta}$ is the $\theta$-component orbital angular momentum, and $S$ is the local spin angular momentum. It can be seen that the local spin $S=\hbar /(2 \mathrm{i}) \cot \theta$ is independent of the wavefunction $\psi$ and thus also independent of the applied potential $V$. Like the proof of the quantization of action variable mentioned in Theorem 3.2, the quantization of the mean value of $L_{\theta}$ can be determined from the contour integral over a closed path $c_{\theta}$ in the $\theta$ complex plane as

$$
\begin{aligned}
\left\langle L_{\theta}\right\rangle & =\frac{1}{2 \pi} \oint_{c_{\theta}} L_{\theta} \mathrm{d} \theta=\frac{\hbar}{2 \pi i} \oint_{c_{\theta}} \frac{1}{\psi} \frac{\partial \psi}{\partial \theta} \mathrm{d} \theta=\frac{\hbar}{2 \pi i} \oint_{c_{\theta}} \frac{1}{\psi} \frac{\mathrm{d} \psi_{\theta}}{\mathrm{d} \theta} \mathrm{d} \theta=\frac{\hbar}{2 \pi i} \oint_{c_{\psi}} \mathrm{d}\left(\ln \psi_{\theta}\right) \\
& =\hbar n_{\theta}, \quad n_{\theta}=0,1,2,3, \cdots
\end{aligned}
$$

where $\psi_{\theta}(\theta)$ is a function of the single variable $\theta$ obtained from $\psi(r, \theta, \phi)$ by treating $r$ and $\phi$ as constants. The function $\psi_{\theta}$ maps the closed path $c_{\theta}$ in the complex $\theta$ plane into a closed path $c_{\psi}$ in the complex $\psi_{\theta}$ plane, and the quantum number $n_{\theta}$ is the number of encirclement of the origin in the $\psi_{\theta}$ plane by the closed path $c_{\psi}$. Analogously, we have the quantization of the $\phi$-component orbital angular momentum as

$$
\begin{aligned}
\left\langle L_{\theta}\right\rangle & =\frac{1}{2 \pi} \oint_{c_{\theta}} L_{\theta} \mathrm{d} \theta=\frac{\hbar}{2 \pi i} \oint_{c_{\theta}} \frac{1}{\psi} \frac{\partial \psi}{\partial \theta} \mathrm{d} \theta=\frac{\hbar}{2 \pi i} \oint_{c_{\theta}} \frac{1}{\psi} \frac{\mathrm{d} \psi}{\mathrm{d} \theta} \mathrm{d} \theta=\frac{\hbar}{2 \pi i} \oint_{c_{\psi}} \mathrm{d}\left(\ln \psi_{\theta}\right) \\
& =\hbar n_{\theta}, \quad n_{\theta}=0,1,2,3, \cdots
\end{aligned}
$$


We now turn the attention to the spin quantization. Using Eq. (7.29), the mean local spin is given by

$$
\langle S\rangle=\frac{1}{2 \pi} \oint_{c_{\theta}} S \mathrm{~d} \theta=\frac{\hbar}{4 \pi i} \oint_{c_{\theta}} \frac{\cos \theta}{\sin \theta} \mathrm{d} \theta .
$$

The evaluation of the above contour integral becomes easier by employing the residue theory as stated in Eq. (4.14), and this leads to

$$
\langle S\rangle=\frac{\hbar}{2} n_{s}, \quad n_{s}=0,1,2,3, \ldots,
$$

where $n_{s}$ is the number of zero of $\sin \theta$, i.e., $n \pi, n \in \mathbf{Z}$, enclosed by the contour $c_{\theta}$, and also note that $\operatorname{since} \sin \theta$ is an analytical function, the number of pole of $\sin \theta$ enclosed by $c_{\theta}$ is always zero. It is worth noting that upon arriving at the result (7.33), we do not specify the type of particles, neither the type of the applied potential. The spin quantization rule (7.33) says that the mean value of the spin is only allowed to be integer multiple of $\hbar / 2$; furthermore, it provides us with a geometrical method to identify the spin of a given particle by inspecting any of its $\theta$ trajectory $c_{\theta}$ and counting the number of the point $n \pi, n \in \mathbf{Z}$ within it. The $\theta$ trajectory can be found by integrating Eq. (6.4).

Referring to the demonstration in Fig. 11, the contour $C_{0}$ encloses no point of $n \pi$, indicating that the particle tracing the contour $C_{0}$ is spinless, while the contour $C_{1}$ encloses one point of $n \pi$, indicating that the particle tracing $C_{1}$ is a spin-1/2 particle. Similarly, the particles tracing $C_{2}$ and $C_{3}$ are spin- 1 and spin-3/2 particles, respectively. The practical computation of the electron trajectory in the hydrogen atom using the Hamilton equations of motion Eq. (6.13) shows that the related trajectory belongs to the type of $C_{1}$ and thus confirms an electron as being a spin-1/2 particle.

The combination of $\hat{P}_{\theta}$ and $\hat{P}_{\phi}$ in Eq. (7.28) affords us the information of total angular momentum.

Lemma 7.2. In spherical coordinates, the total angular momentum operator is given by

$$
\hat{\mathbf{J}}^{2}=\hat{P}_{\theta}^{2}+\frac{\hat{P}_{\phi}^{2}}{\sin ^{2} \theta}=\hat{\mathbf{L}}^{2}+(\hbar / 2)^{2}+\frac{(\hbar / 2)^{2}}{\sin ^{2} \theta},
$$

where $\hat{\mathbf{L}}^{2}$ is the usual orbit angular momentum operator defined in Eq. (6.7).

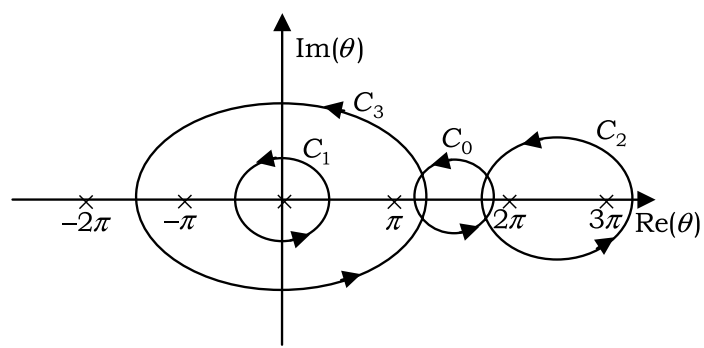

Fig. 11. The quantization of spin is characterized by the number of $n \pi, n \in \mathbf{Z}$, enclosed by the $\theta$ complex trajectories. In the figure, the contour $C_{0}$ encloses no point of $n \pi$, indicating that the particle tracing the contour $C_{0}$ is a spinless particle, while the contour $C_{1}$ encloses one point of $n \pi$, indicating that the particle tracing $C_{1}$ is a spin-1/2 particle. Similarly, the particles tracing $C_{2}$ and $C_{3}$ are spin-1 and spin-3/2 particles, respectively. 
Proof. The combination of the $\theta$-component and the $\phi$-component angular momenta gives the total angular momentum vector as

$$
\mathbf{J}=P_{\theta} \mathbf{e}_{\theta}+\frac{P_{\phi}}{\sin \theta} \mathbf{e}_{\phi}=P_{\theta} \mathbf{e}_{\theta}+\frac{L_{z}}{\sin \theta} \mathbf{e}_{\phi},
$$

which has the operator representation:

$$
\hat{\mathbf{J}}=\hat{P}_{\theta} \mathbf{e}_{\theta}+\frac{\hat{L}_{z}}{\sin \theta} \mathbf{e}_{\phi}
$$

where $\mathbf{e}_{\theta}$ and $\mathbf{e}_{\phi}$ are, respectively, the unit vectors in the $\theta$ and $\phi$ directions. Using the definitions of $\hat{P}_{\theta}$ and $\hat{P}_{\phi}=\hat{L}_{z}$ from Eq. (7.28), the operation represented by $\hat{\mathbf{J}}^{2}$ becomes

$$
\hat{\mathbf{J}}^{2} \psi=(\hat{\mathbf{J}} \cdot \hat{\mathbf{J}}) \psi=\hat{P}_{\theta}^{2} \psi+\frac{1}{\sin ^{2} \theta} \hat{P}_{\phi}^{2} \psi,
$$

where $\hat{P}_{\theta}^{2} \psi$ and $\hat{P}_{\phi}^{2} \psi$ are evaluated, respectively, as

$$
\begin{aligned}
\hat{P}_{\theta}^{2} \psi & =\hat{P}_{\theta}\left(\hat{P}_{\theta} \psi\right)=\left(\frac{\hbar}{\mathrm{i}}\right)^{2}\left(\frac{\partial}{\partial \theta}+\frac{\cot \theta}{2}\right)\left(\frac{\partial \psi}{\partial \theta}+\frac{\cot \theta}{2} \psi\right) \\
& =-\hbar^{2}\left(\frac{\partial^{2} \psi}{\partial \theta^{2}}+\cot \theta \frac{\partial \psi}{\partial \theta}\right)+\left[(\hbar / 2)^{2}+\frac{(\hbar / 2)^{2}}{\sin ^{2} \theta}\right] \psi, \\
\hat{P}_{\phi}^{2} \psi & =\left(\frac{\hbar}{\mathrm{i}} \frac{\partial}{\partial \phi}\right)\left(\frac{\hbar}{\mathrm{i}} \frac{\partial \psi}{\partial \phi}\right)=-\hbar^{2} \frac{\partial^{2} \psi}{\partial \phi^{2}} .
\end{aligned}
$$

The substitution of $\hat{P}_{\theta}^{2}$ and $\hat{P}_{\phi}^{2}$ into Eq. (7.37) gives rise to the representation of $\hat{\mathbf{J}}^{2}$ as

$$
\hat{\mathbf{J}}^{2}=-\hbar^{2}\left(\frac{\partial^{2}}{\partial \theta^{2}}+\cot \theta \frac{\partial}{\partial \theta}+\frac{1}{\sin ^{2} \theta} \frac{\partial^{2}}{\partial \phi^{2}}\right)+\left[(\hbar / 2)^{2}+\frac{(\hbar / 2)^{2}}{\sin ^{2} \theta}\right],
$$

where the first three terms in $\hat{\mathbf{J}}^{2}$ is the orbital angular momentum operator $\hat{\mathbf{L}}^{2}$ already derived in Eq. (6.7) and the remaining two terms are due to spin motion.

\section{Quantum Hamilton mechanics in electromagnetic field}

Analogous to classical mechanics, the extension of the quantum Hamiltonian (7.12) to include the action of electromagnetic field has the following form:

$$
H=\frac{g^{j k}}{2 m}\left(p_{k}-\frac{e}{c} A_{k}\right)\left(p_{j}-\frac{e}{c} A_{j}\right)-\frac{\hbar^{2}}{2 m} g^{j k} \frac{\partial^{2} \ln \psi}{\partial q^{j} \partial q^{k}}+\frac{\hbar}{2 m \mathrm{i}} \frac{p_{k}}{\sqrt{g}} \frac{\partial}{\partial q^{j}}\left(\sqrt{g} g^{j k}\right)+e \Phi(\mathbf{q}, t),
$$

where the vector potential $\mathbf{A}(\mathbf{q}, t)$ and the scalar potential $\Phi(\mathbf{q}, t)$ are related to the magnetic and the electric field strength according to

$$
\mathbf{E}=-\nabla \Phi-\frac{1}{c} \frac{\partial \mathbf{A}}{\partial t}, \quad \mathbf{B}=\nabla \times \mathbf{A}
$$

The electromagnetic potentials $\mathbf{A}$ and $\phi$ are gauge dependent; particularly in Coulomb gauge, we have

$$
\nabla \cdot \mathbf{A}=\partial_{j} A^{j}=\partial^{j} A_{j}=0
$$


By treating $q^{k}$ and $p_{k}$ as independent variables in Eq. (8.1), the Hamilton equations of motion are obtained as:

$$
\begin{aligned}
\frac{\mathrm{d} q^{k}}{\mathrm{~d} t} & =\frac{\partial H}{\partial p_{k}}=\frac{g^{j k}}{m}\left(p_{j}-\frac{e}{c} A_{j}\right)+\frac{\hbar}{2 m \mathrm{i}} \frac{1}{\sqrt{g}} \frac{\partial}{\partial q^{j}}\left(\sqrt{g} g^{j k}\right), \\
\frac{\mathrm{d} p_{k}}{\mathrm{~d} t} & =-\frac{\partial H}{\partial q^{k}} .
\end{aligned}
$$

If we search the solution for $p_{k}$ in the form of $p_{k}=\partial_{k} S=\partial S / \partial q^{k}$, it can be shown, by a similar way leading to Theorem 7.1, that Eq. (8.4) is satisfied, if $S$ is the solution of the following quantum Hamilton-Jacobi equation:

$$
\frac{\partial S}{\partial t}+\frac{1}{2 m}\left(\partial_{k} S-\frac{e}{c} A_{k}\right)\left(\partial^{k} S-\frac{e}{c} A^{k}\right)+\frac{\hbar}{2 m \mathrm{i}} \partial_{k} \partial^{k} S+e \Phi=0,
$$

where the covariant derivative $\partial_{k}$ and the contravariant derivative $\partial^{k}$ have been defined in Eqs. (7.9) and (7.10). The quantum Hamilton-Jacobi equation (8.5) is reduced to the Schrödinger equation via the transformation $S=(\hbar / \mathrm{i}) \ln \psi$ :

$$
\mathrm{i} \hbar \frac{\partial \psi}{\partial t}=\left[\frac{1}{2 m}\left(\frac{\hbar}{\mathrm{i}} \partial_{k}-\frac{e}{c} A_{k}\right)\left(\frac{\hbar}{\mathrm{i}} \partial^{k}-\frac{e}{c} A^{k}\right)+e \Phi\right] \psi,
$$

where the Coulomb gauge $\partial_{k} A^{k}=0$ has been applied in the above derivation. On the other hand, we can find the Hamiltonian operator $\hat{H}$ from Eq. (8.1) by inserting $p_{k}=\partial_{k} S=-\mathrm{i} \hbar \partial_{k}(\ln \psi)$ and comparing the result with the definition $H=(1 / \psi) \hat{H} \psi$ :

$$
H=\frac{1}{\psi}\left[\frac{1}{2 m}\left(\frac{\hbar}{\mathrm{i}} \partial_{k}-\frac{e}{c} A_{k}\right)\left(\frac{\hbar}{\mathrm{i}} \partial^{k}-\frac{e}{c} A^{k}\right)+e \Phi\right] \psi=\frac{1}{\psi} \hat{H} \psi,
$$

from which $\hat{H}$ can be identified as

$$
\hat{H}=\frac{1}{2 m}\left(\frac{\hbar}{\mathrm{i}} \partial_{k}-\frac{e}{c} A_{k}\right)\left(\frac{\hbar}{\mathrm{i}} \partial^{k}-\frac{e}{c} A^{k}\right)+e \Phi,
$$

and Eq. (8.6) turns out to be the familiar operator form:

$$
\mathrm{i} \hbar \frac{\partial \psi}{\partial t}=\hat{H} \psi
$$

In comparison with the Hamiltonian $H_{\mathrm{c}}$ for classical electrodynamics expressed in the curvilinear coordinates $q^{j}$ :

$$
H_{\mathrm{c}}=\frac{1}{2 m}\left(p_{k}-\frac{e}{c} A_{k}\right)\left(p^{k}-\frac{e}{c} A^{k}\right)+e \Phi
$$

it is interesting to find that the quantum Hamiltonian $\hat{H}$ can be obtained from $H_{\mathrm{c}}$ by simply replacing the canonical covariant momentum $p_{k}$ with the covariant derivative $-\mathrm{i} \hbar \partial_{k}$ and the canonical contravariant momentum $p^{k}$ with the contravariant derivative $-\mathrm{i} \hbar \partial^{k}$. Hence, the role of the quantization axiom $p \rightarrow-\mathrm{i} \hbar \nabla$ in Cartesian coordinates is now superseded by the correspondence $p_{k} \rightarrow-\mathrm{i} \hbar \partial_{k}$ and $p^{k} \rightarrow-\mathrm{i} \hbar \partial^{k}$ in the curvilinear coordinates.

As for the operator $\hat{P}_{k}$ corresponding to the mechanical momentum $P_{k}$, we use Eq. (8.4a) in Eq. (7.25) to obtain $P_{k}$ as

$$
\begin{aligned}
P_{j} & =m g_{j k} \dot{q}^{k}=m g_{j k}\left[\frac{g^{n k}}{m}\left(p_{n}-\frac{e}{c} A_{n}\right)+\frac{\hbar}{2 m \mathrm{i}} \frac{1}{\sqrt{g}} \frac{\partial}{\partial q^{n}}\left(\sqrt{g} g^{n k}\right)\right] \\
& =p_{j}-\frac{e}{c} A_{j}+\frac{\hbar}{2 \mathrm{i}} \frac{g_{j k}}{\sqrt{g}} \frac{\partial}{\partial q^{n}}\left(\sqrt{g} g^{n k}\right),
\end{aligned}
$$


where we have applied the relation that $g_{j k} g^{n k}=\delta_{j}^{n}=1$ if $j=n$, otherwise, $g_{j k} g^{n k}=0$. Substituting the canonical momentum $p_{j}=-\mathrm{i} \hbar \partial \ln \psi / \partial q^{j}$ into Eq. (8.11), we obtain

$$
P_{j}=\frac{\hbar}{\mathrm{i} \psi} \frac{\partial \psi}{\partial q^{j}}-\frac{e}{c} A_{j}+\frac{\hbar}{2 \mathrm{i}} \frac{g_{j k}}{\sqrt{g}} \frac{\partial}{\partial q^{n}}\left(\sqrt{g} g^{n k}\right)=\frac{1}{\psi}\left[\frac{\hbar}{\mathrm{i}} \frac{\partial}{\partial q^{j}}-\frac{e}{c} A_{j}+\frac{\hbar}{2 \mathrm{i}} \frac{g_{j k}}{\sqrt{g}} \frac{\partial}{\partial q^{n}}\left(\sqrt{g} g^{n k}\right)\right] \psi .
$$

The comparison with the definition $P_{j}=(1 / \psi) \hat{P}_{j} \psi$ gives

$$
\hat{P}_{j}=\frac{\hbar}{\mathrm{i}}\left[\frac{\partial}{\partial q^{j}}+\frac{1}{2} \frac{g_{j k}}{\sqrt{g}} \frac{\partial}{\partial q^{n}}\left(\sqrt{g} g^{n k}\right)\right]-\frac{e}{c} A_{j} .
$$

This expression of momentum operator is valid for any curvilinear coordinate system with any number of generalized coordinates $q^{j}$. For the special case of $A_{j}=0$ and under the orthogonal coordinate system with $g_{j k}$ given by Eq. (7.22), Eq. (8.12) reduces automatically to Eq. (7.26). The following example illustrates how to derive mechanical momentum operator from the Hamilton equations (8.4a) under spherical coordinates.

Example 8.1. In spherical coordinates, we have $q^{1}=r, q^{2}=\theta, q^{3}=\phi, g_{11}=1 / g^{11}=1$, $g_{22}=1 / g^{22}=r^{2}, g_{33}=1 / g^{33}=r^{2} \sin ^{2} \theta$, and $g_{j k}=0, j \neq k$. With the above substitutions, Eq. (8.4a) reduces to

$$
\begin{aligned}
& \dot{r}=\frac{\partial H}{\partial p_{r}}=\frac{1}{m}\left(p_{r}-\frac{e}{c} A_{r}\right)+\frac{\hbar}{\mathrm{i} m r}, \\
& \dot{\theta}=\frac{\partial H}{\partial p_{\theta}}=\frac{1}{m r}\left(\frac{1}{r} p_{\theta}-\frac{e}{c} A_{\theta}\right)+\frac{\hbar}{2 m \mathrm{i}} \frac{\cot \theta}{r^{2}}, \\
& \dot{\phi}=\frac{\partial H}{\partial p_{\phi}}=\frac{1}{m r \sin \theta}\left(\frac{1}{r \sin \theta} p_{\phi}-\frac{e}{c} A_{\phi}\right),
\end{aligned}
$$

from which the mechanical momenta can be derived as

$$
\begin{aligned}
& P_{r}=m \dot{r}=p_{r}-\frac{e}{c} A_{r}+\frac{\hbar}{\mathrm{i} r}, \\
& P_{\theta}=m r^{2} \dot{\theta}=p_{\theta}-\frac{e}{c} r A_{\theta}+\frac{\hbar}{2 \mathrm{i}} \cot \theta, \\
& P_{\phi}=m r^{2} \dot{\phi} \sin ^{2} \theta=p_{\phi}-\frac{e}{c} r A_{\phi} \sin \theta .
\end{aligned}
$$

Recalling that the canonical momenta $p_{r}, p_{\theta}$, and $p_{\phi}$ are related to wavefunction $\psi$ as in Eq. (6.2), we can express the mechanical momenta in terms of $\psi$ and then obtain their associated operators from the definition in Eq. (3.1):

$$
\begin{aligned}
P_{r}= & \frac{1}{\psi}\left[\frac{\hbar}{\mathrm{i}}\left(\frac{\partial}{\partial r}+\frac{\hbar}{r}\right)-\frac{e}{c} A_{r}\right] \psi=\frac{1}{\psi} \hat{P}_{r} \psi \Rightarrow \hat{P}_{r}=\frac{\hbar}{\mathrm{i}}\left(\frac{\partial}{\partial r}+\frac{\hbar}{r}\right)-\frac{e}{c} A_{r}, \\
P_{\theta}= & \frac{1}{\psi}\left[\frac{\hbar}{\mathrm{i}}\left(\frac{\partial}{\partial \theta}+\frac{\cot \theta}{2}\right)-\frac{e}{c} r A_{\theta}\right] \psi=\frac{1}{\psi} \hat{P}_{\theta} \psi \\
& \Rightarrow \hat{P}_{\theta}=\frac{\hbar}{\mathrm{i}}\left(\frac{\partial}{\partial \theta}+\frac{\cot \theta}{2}\right)-\frac{e}{c} r A_{\theta}, \\
P_{\phi}= & \frac{1}{\psi}\left[\frac{\hbar}{\mathrm{i}} \frac{\partial}{\partial \phi}-\frac{e}{c} r A_{\phi} \sin \theta\right] \psi \Rightarrow \hat{P}_{\phi}=\frac{\hbar}{\mathrm{i}} \frac{\partial}{\partial \phi}-\frac{e}{c} r A_{\phi} \sin \theta .
\end{aligned}
$$

The above results can also be obtained directly from the formula (8.12). 
We have learned from Lemma 7.2 that the operator $\hat{P}_{\theta}$ contains the effect of spin motion. Here, we wish to study the spin effect by constructing a total Hamiltonian operator from $\hat{P}_{r}, \hat{P}_{\theta}$, and $\hat{P}_{\phi}$.

Lemma 8.1. A total Hamiltonian operator comprising both orbit and spin motion is given by

$$
\hat{H}_{\text {Total }}=\frac{1}{2 m}\left[\hat{P}_{r}^{2}+\frac{1}{r^{2}}\left(\hat{P}_{\theta}^{2}+\frac{1}{\sin ^{2} \theta} \hat{P}_{\phi}^{2}\right)\right]+e \phi=\hat{H}+\frac{1}{2 m r^{2}}\left[(\hbar / 2)^{2}+\frac{(\hbar / 2)^{2}}{\sin ^{2} \theta}\right],
$$

where $\hat{H}$ is the usual Hamiltonian operator defined in Eq. (6.8).

Proof. $\hat{H}_{\text {Total }}$ is the operator associated with the local observable

$$
H_{\text {Total }}=\frac{1}{2 m}\left[P_{r}^{2}+\frac{1}{r^{2}}\left(P_{\theta}^{2}+\frac{1}{\sin ^{2} \theta} P_{\phi}^{2}\right)\right]+e \Phi .
$$

We call $\hat{H}_{\text {Total }}$ the total Hamiltonian operator because it describes both orbit and spin motions, whereas the Hamiltonian operator $\hat{H}$ defined in Eq. (6.8) involves only orbit motion. Making use of the momentum operators derived in Eq. (8.15), we can find the total Hamiltonian $\hat{H}_{\text {Total }}$ as

$$
\begin{aligned}
\hat{H}_{\text {Total }}= & \frac{1}{2 m}\left[\hat{P}_{r}^{2}+\frac{1}{r^{2}}\left(\hat{P}_{\theta}^{2}+\frac{1}{\sin ^{2} \theta} \hat{P}_{\phi}^{2}\right)\right]+e \Phi \\
= & {\left[\frac{1}{2 m} \hat{P}_{r}^{2}+\frac{1}{2 m r^{2}} \hat{L}^{2}+e \Phi\right]+\frac{1}{2 m r^{2}}\left[(\hbar / 2)^{2}+\frac{(\hbar / 2)^{2}}{\sin ^{2} \theta}\right] } \\
& -\frac{\hbar}{2 m \mathrm{i}} \frac{e}{c}\left[\frac{2}{r} A_{r}+\frac{\partial A_{r}}{\partial r}+\frac{\cot \theta}{r} A_{\theta}+\frac{1}{r} \frac{\partial A_{\theta}}{\partial \theta}+\frac{1}{r \sin \theta} \frac{\partial A_{\phi}}{\partial \phi}\right] .
\end{aligned}
$$

The terms within the first pair of brackets just form the Hamiltonian $\hat{H}$ already defined in Eq. (6.8), and the terms within the third pair of brackets constitute $\nabla \cdot \mathbf{A}$ in spherical coordinates, which is zero due to Coulomb gauge mentioned in Eq. (8.3). With the above simplifications, Eq. (8.18) then reduces to the succinct result in Eq. (8.16), where the total Hamiltonian $H_{\text {Total }}$ comprises the energy $H$ due to orbit motion and the energy due to spin motion.

In the above discussion, we have demonstrated how to drive electrodynamics operators from the Hamilton equations of motion (8.4a), but till now we have not exploited the usefulness of Eq. (8.4b). The right-hand side of Eq. (8.4b) is the generalized force associated with the generalized coordinate $q^{k}$. This generalized force is composed of the Lorentz force and the quantum force. To clarify the constitution of the generalized force, we shall focus on a three-dimensional orthogonal coordinate system $\left(q^{1}, q^{2}, q^{3}\right)$ under which Lorentz force has a succinct expression. In terms of the scalar potential $\Phi$ and the vector potential A, Lorentz force $\mathbf{f}_{\mathrm{L}}$ has the following expression:

$$
\mathbf{f}_{\mathrm{L}}=e \mathbf{E}+\frac{e}{c} \dot{\mathbf{r}} \times \mathbf{B}=-e\left(\nabla \Phi+\frac{1}{c} \frac{\partial \mathbf{A}}{\partial t}\right)+\frac{e}{c} \dot{\mathbf{r}} \times(\nabla \times \mathbf{A}) .
$$

Let $\mathbf{e}_{k}$ be the unit vector of the coordinate $q^{k}$. The expansion of $\nabla \Phi$ and $\nabla \times \mathbf{A}$ with respect to $\mathbf{e}_{k}$ yields the following results:

$$
\mathbf{A}=\frac{A_{1}}{h_{1}} \mathbf{e}_{1}+\frac{A_{2}}{h_{2}} \mathbf{e}_{2}+\frac{A_{3}}{h_{3}} \mathbf{e}_{3},
$$




$$
\begin{aligned}
& \nabla \Phi=\frac{1}{h_{1}} \frac{\partial \Phi}{\partial q^{1}} \mathbf{e}_{1}+\frac{1}{h_{2}} \frac{\partial \Phi}{\partial q^{2}} \mathbf{e}_{2}+\frac{1}{h_{3}} \frac{\partial \Phi}{\partial q^{3}} \mathbf{e}_{3} \\
& \nabla \times \mathbf{A}=\frac{1}{h_{1} h_{2} h_{3}}\left[\left(\frac{\partial A_{3}}{\partial q^{2}}-\frac{\partial A_{2}}{\partial q^{3}}\right) h_{1} \mathbf{e}_{1}+\left(\frac{\partial A_{1}}{\partial q^{3}}-\frac{\partial A_{3}}{\partial q^{1}}\right) h_{2} \mathbf{e}_{2}+\left(\frac{\partial A_{2}}{\partial q^{1}}-\frac{\partial A_{1}}{\partial q^{2}}\right) h_{3} \mathbf{e}_{3}\right] .
\end{aligned}
$$

Substituting Eq. (8.20) and $\dot{\mathbf{r}}$ from Eq. (7.23) into Eq. (8.19), we obtain the $k$ th component of the Lorentz force $\mathbf{f}_{\mathrm{L}}$ as

$$
\begin{aligned}
\mathbf{f}_{\mathrm{L}, k} & =\left(e \mathbf{E}+\frac{e}{c} \dot{\mathbf{r}} \times \mathbf{B}\right)_{k}=-\frac{e}{h_{k}}\left(\frac{\partial \Phi}{\partial q^{k}}+\frac{1}{c} \frac{\partial A_{k}}{\partial t}\right)+\frac{e}{c} \sum_{j=1}^{3} \frac{\dot{q}^{j}}{h_{k}}\left(\frac{\partial A_{j}}{\partial q^{k}}-\frac{\partial A_{k}}{\partial q^{j}}\right), \\
k & =1,2,3 .
\end{aligned}
$$

With the above preparation, we are going to analyze the force composition within Eq. (8.4b). First, we rewire the Hamiltonian (8.1) under orthogonal coordinate system $\left(q^{1}, q^{2}, q^{3}\right)$ as

$$
H=\frac{1}{2 m} \sum_{j=1}^{3} \frac{1}{h_{j}^{2}}\left[\left(p_{j}-\frac{e}{c} A_{j}\right)^{2}+\frac{\hbar}{\mathrm{i}} p_{j} \frac{\partial \ln H_{j}}{\partial q^{j}}\right]+V_{\psi}+V_{\phi},
$$

where $H_{k}$ is the abbreviation of $h_{1} h_{2} h_{3} / h_{k}^{2}, V_{\phi}$ is the electric potential $e \Phi$, and $V_{\psi}$ is the quantum potential due to wavefunction $\psi$ :

$$
V_{\psi}=-\frac{\hbar^{2}}{2 m} \sum_{j=1}^{3} \frac{1}{h_{j}^{2}} \frac{\partial^{2} \ln \psi}{\partial q^{j} \partial q^{j}} .
$$

Notice that if all the terms containing Planck constant $\hbar$ are neglected, Eq. (8.22) reduces to the Hamiltonian for classical electrodynamics. The next step to derive the force composition in Eq. (8.4b) is to eliminate the canonical momentum $p_{j}$ between Eqs. (8.4b) and (8.4a). From Eq. (8.4a), we can express $p_{k}$ in terms of $q^{k}$ as

$$
p_{k}=m h_{k}^{2} \dot{q}^{k}+\frac{e}{c} A_{k}-\frac{\hbar}{2 \mathrm{i}} \frac{\partial \ln H_{k}}{\partial q^{k}}, \quad k=1,2,3,
$$

whose derivative with respect to time leads to

$$
\dot{p}_{k}=m h_{k}^{2} \ddot{q}^{k}+\sum_{j=1}^{3} m \dot{q}^{j} \dot{q}^{k} \frac{\partial h_{j}^{2}}{\partial q^{j}}+\frac{e}{c} \frac{\partial A_{k}}{\partial t}+\frac{e}{c} \sum_{j=1}^{3} m \dot{q}^{j} \frac{\partial A_{k}}{\partial q^{j}}-\frac{\hbar}{2 \mathrm{i}} \sum_{j=1}^{3} \dot{q}^{j} \frac{\partial^{2} \ln H_{k}}{\partial q^{j} \partial q^{k}} .
$$

The substitution of $\dot{p}_{k}$ from Eq. (8.25) and $H$ from Eq. (8.22) into Eq. (8.4b) yields a Newtonian-like equations of motion, which involves only time derivatives of $q^{k}$ :

$$
\begin{aligned}
& m h_{k} \ddot{q}^{k}+2 m \sum_{j=1}^{3} \dot{q}^{k} \dot{q}^{j} \frac{\partial h_{k}}{\partial q^{j}}-m \sum_{j=1}^{3} \frac{h_{j}}{h_{k}}\left(\dot{q}^{j}\right)^{2} \frac{\partial h_{j}}{\partial q^{k}}+\frac{\hbar}{2 \mathrm{i} h_{k}} \sum_{j=1}^{3} \dot{q}^{j} \frac{\partial^{2} \ln \left(h_{k} / h_{j}\right)}{\partial q^{j} \partial q^{k}} \\
& =\mathbf{f}_{\mathrm{L}, k}+\mathbf{f}_{h, k}+\mathbf{f}_{A, k}+\mathbf{f}_{\psi, k} .
\end{aligned}
$$

It can be seen that there are four force terms in the right-hand side of Eq. (8.26). The force $\mathbf{f}_{\mathrm{L}, k}$ is the $k$ th component of the Lorentz force as defined in Eq. (8.21). The force $\mathbf{f}_{h, k}$ stems from the metric tensor of the considered coordinate system: 


$$
f_{h, k}=-\frac{1}{h_{k}} \frac{\partial}{\partial q^{k}} V_{h}=-\frac{1}{h_{k}} \frac{\partial}{\partial q^{k}}\left[\frac{\hbar^{2}}{8 m} \sum_{j=1}^{3} \frac{1}{h_{j}^{2}}\left(\frac{\partial \ln H_{j}}{\partial q^{j}}\right)^{2}\right] .
$$

The force $\mathbf{f}_{A, k}$ is caused solely by the vector potential $\mathbf{A}$ :

$$
f_{A, k}=-\frac{1}{h_{k}} \frac{\partial}{\partial q^{k}} V_{A}=-\frac{1}{h_{k}} \frac{\partial}{\partial q^{k}}\left[\frac{\hbar}{2 m \mathrm{i}} \frac{e}{c} \sum_{j=1}^{3} \frac{A_{j}}{h_{j}^{2}} \frac{\partial \ln H_{j}}{\partial q^{j}}\right] .
$$

The force $\mathbf{f}_{\psi, k}$ is due to the wavefunction $\psi$ :

$$
f_{\psi, k}=-\frac{1}{h_{k}} \frac{\partial}{\partial q^{k}} V_{\psi}=-\frac{1}{h_{k}} \frac{\partial}{\partial q^{k}}\left[\frac{\hbar^{2}}{2 m} \sum_{j=1}^{3} \frac{1}{h_{j}^{2}} \frac{\partial^{2} \ln \psi}{\partial q^{j} \partial q^{j}}\right] .
$$

Except for the Lorentz force $\mathbf{f}_{\mathrm{L}, k}$, the other three forces $\mathbf{f}_{h, k}, \mathbf{f}_{A, k}$, and $\mathbf{f}_{A, k}$ are all due to quantum effect. Furthermore, the three quantum forces are derived, respectively, from their associated quantum potentials $V_{h}, V_{A}$, and $V_{\psi}$. The quantum force $\mathbf{f}_{A, k}$ caused by the vector potential $\mathbf{A}$ needs particular attention. It is generally assumed that only the magnetic field $\mathbf{B}$ is physically active and that the vector potential $\mathbf{A}$, from which the field is derived via $\mathbf{B}=\nabla \times \mathbf{A}$, has itself no further physical significance beyond that of being a mathematic auxiliary in the description of the field. However, it can be seen from Eq. (8.26) that there exists a quantum force $\mathbf{f}_{A, k}$ that is directly related to the vector potential A. The quantum force $\mathbf{f}_{A, k}$ is existent, even though the magnetic field $\mathbf{B}=\nabla \times \mathbf{A}$ is zero. The influence of the vector potential on charged particle had been verified experimentally and was known as Aharonov-Bohm effect [12]. Eq. (8.26) can be conceived of as a quantitative description of Aharonov-Bohm effect and provides the detailed mechanism explaining the motion of a charged particle under the action of the vector potential.

Example 8.2. We consider the explicit expression of Eq. (8.26) in spherical coordinates. With the same notation used in Example 7.1, Eq. (8.26) becomes

$$
\begin{aligned}
& m \ddot{r}-m r \dot{\theta}^{2}-m r \dot{\phi}^{2} \sin ^{2} \theta=\left(e \mathbf{E}+\frac{e}{c} \dot{\mathbf{r}} \times \mathbf{B}\right)_{r}-\frac{\partial}{\partial r}\left(V_{h}+V_{A}+V_{\psi}\right), \\
& m r \ddot{\theta}+2 m \dot{r} \dot{\theta}-m r \dot{\phi}^{2} \sin \theta \cos \theta=\left(e \mathbf{E}+\frac{e}{c} \dot{\mathbf{r}} \times \mathbf{B}\right)_{\theta}-\frac{1}{r} \frac{\partial}{\partial \theta}\left(V_{h}+V_{A}+V_{\psi}\right), \\
& m r \ddot{\phi} \sin \theta+2 m \dot{r} \dot{\phi} \sin \theta+2 m r \dot{\phi} \dot{\theta} \cos \theta \\
& \quad=\left(e \mathbf{E}+\frac{e}{c} \dot{\mathbf{r}} \times \mathbf{B}\right)_{\phi}-\frac{1}{r \sin \theta} \frac{\partial}{\partial \phi}\left(V_{h}+V_{A}+V_{\psi}\right),
\end{aligned}
$$

where the three potentials $V_{h}, V_{A}$, and $V_{\psi}$ are found from Eq. (8.27) as

$$
\begin{aligned}
& V_{h}=\frac{\hbar^{2}}{8 m r^{2}}\left(4+\cot ^{2} \theta\right), \quad V_{A}=\frac{\hbar}{m \mathrm{i}} \frac{e}{c}\left(\frac{A_{r}}{r}+\frac{\cot \theta}{2 r^{2}} A_{\theta}\right), \\
& V_{\psi}=-\frac{\hbar^{2}}{2 m}\left(\frac{\partial^{2} \ln \psi}{\partial r^{2}}+\frac{1}{r^{2}} \frac{\partial^{2} \ln \psi}{\partial \theta^{2}}+\frac{1}{r^{2} \sin ^{2} \theta} \frac{\partial^{2} \ln \psi}{\partial \phi^{2}}\right) .
\end{aligned}
$$

The wavefunction $\psi$ is a solution of the Schrödinger equation (8.6) for the given scalar potential $\Phi$ and the vector potential A. Hence, the motion of a charged particle in the quantum state $\psi$ under the action of the electromagnetic potentials $\Phi$ and $\mathbf{A}$ can be found by the 
integration of Eq. (8.28). Indeed, the first integration of Eq. (8.28) has been obtained in Eq. (8.13) with the canonical momenta given by Eq. (7.9):

$$
\begin{aligned}
& \dot{r}=\frac{1}{m}\left(\frac{\hbar}{\mathrm{i}} \frac{\partial \ln \psi}{\partial r}-\frac{e}{c} A_{r}\right)+\frac{\hbar}{\mathrm{i} m r}, \\
& \dot{\theta}=\frac{1}{m r}\left(\frac{\hbar}{\mathrm{i}} \frac{1}{r} \frac{\partial \ln \psi}{\partial \theta}-\frac{e}{c} A_{\theta}\right)+\frac{\hbar}{2 m \mathrm{i}} \frac{\cot \theta}{r^{2}}, \\
& \dot{\phi}=\frac{1}{m r \sin \theta}\left(\frac{\hbar}{\mathrm{i}} \frac{1}{r \sin \theta} \frac{\partial \ln \psi}{\partial \phi}-\frac{e}{c} A_{\phi}\right) .
\end{aligned}
$$

The integration of this set of first-order ODE's tells us how the particle's trajectory $(r(t), \theta(t), \phi(t))$ responses to the change of the vector potential $\mathbf{A}=\left(A_{r}, A_{\theta}, A_{\phi}\right)$ and thus provides us with a visualization of Aharonov-Bohm effect. In the absence of vector potential $\mathbf{A}$, we can combine the electric force $e E=-\nabla V_{\phi}=-e \nabla \Phi$ with the other three quantum force and express the right-hand sides of Eq. (8.28) as the gradient of a total potential, $-\nabla V_{\text {Total }}$, where

$$
\begin{aligned}
V_{\text {Total }} & =V_{h}+V_{\psi}+V_{\phi} \\
& =\frac{\hbar^{2}}{8 m r^{2}}\left(4+\cot ^{2} \theta\right)-\frac{\hbar^{2}}{2 m}\left(\frac{\partial^{2} \ln \psi}{\partial r^{2}}+\frac{1}{r^{2}} \frac{\partial^{2} \ln \psi}{\partial \theta^{2}}+\frac{1}{r^{2} \sin ^{2} \theta} \frac{\partial^{2} \ln \psi}{\partial \phi^{2}}\right)+e \Phi .
\end{aligned}
$$

This total potential is the exact one that we have encountered in hydrogen atom as shown in Eq. (6.15).

\section{Conclusions}

Due to the complex-valued nature of Schrödinger equation, analytical mechanics compatible with Schrödinger equation need be extended to complex domain such that all the canonical variables and the generalized forces describing a particle's motion become complex-valued. The complex extension allows us to treat classical mechanics and quantum mechanics integrally by using the existing methods developed in analytical mechanics. In this paper, complex-extended Hamilton equations of motion are derived from a complex Hamiltonian stemmed from Schrödinger equation. We then exploit this set of complex Hamilton equations to derive quantum operators under any curvilinear coordinate system and to describe various quantum phenomena by using the well-known methods of analytical mechanics. The Hamilton-mechanical approach to deriving quantum operator releases two limitations encountered in the quantization axiom $\mathbf{p} \rightarrow \hat{\mathbf{p}}=\mathrm{i} \hbar \nabla$, i.e., the limitation to Cartesian coordinates and the limitation to observables having classical counterpart. We point out in the paper that for each quantum operator $\hat{A}$ in quantum mechanics, there corresponds a physical entity $A(\mathbf{q}, \mathbf{p})$ in the complex-extended Hamilton mechanics. Knowing the expression for $A(\mathbf{q}, \mathbf{p})$ with $\mathbf{q}$ and $\mathbf{p}$ satisfying the complex Hamilton equations permits us to deduce $\hat{A}$ readily, regardless of the coordinate system being used. The equivalence between the operator $\hat{A}$ and its related complex function $A(\mathbf{q}, \mathbf{p})$ implies that the quantum systems having been described by the eigenvalue problem of $\hat{A}$ now can be described by the complex Hamilton equations of motion governing $A(\mathbf{q}, \mathbf{p})$. This equivalence yields a new method of solving quantum problems and we have verified its validity by showing hat the various quantum phenomena such as quantization, 
tunneling, atomic shell structure, spin, and Aharonov-Bohm effect can all be predicted correctly.

\section{References}

[1] H. Goldstein, Classical Mechanics, Addison-Wesley, Reading, MA, 1980 (Chapter 10).

[2] R.A. Leacock, M.J. Padgett, Phys. Rev. Lett. 50 (1983) 3; Phys. Rev. D 28 (1983) 2491.

[3] R.S. Bhalla, A.K. Kapoor, P.K. Panigrahi, Am. J. Phys. 65 (1997) 1187;

S.S. Ranjani, K.G. Geojo, A.K. Kapoor, P.K. Panigrahi, Mod. Phys. Lett. A 19 (2004) 1457.

[4] D. Bohm, Phys. Rev. 85 (166) (1952) 180.

[5] R.P. Feynman, Lectures on Physics, Addison-Wesley, Reading, MA, 1965 (Chapter 21).

[6] L.L. Pennisi, L.I. Gordon, S. Lasher, Elements of Complex Variables, Holt, Rinehart and Winston, New York, 1976, p. 293;

W. Rudin, Real and Complex Analysis, McGraw-Hill, Singapore, 1986, p. 225.

[7] C.D. Yang, Chaos Solitons Fractals 30 (2006) 342.

[8] J.G. Muga, R.S. Mayato, I.L. Egusquiza (Eds.), Time in Quantum Mechanics, Springer, Berlin, 2002.

[9] C.D. Yang, Ann. Phys. 319 (2005) 444.

[10] C.D. Yang, Ann. Phys. 319 (2005) 399.

[11] C.D. Yang, Int. J. Quantum Chem. 106 (2006) 1620.

[12] Y. Aharonov, D. Bohm, Phys. Rev. 115 (1959) 485. 